\title{
Fitting of circles and ellipses Least squares solution
}

\section{Report}

\section{Author(s):}

Strebel, Rolf; Gander, Walter; Golub, Gene Howard

Publication date:

1994-06

Permanent link:

https://doi.org/10.3929/ethz-a-000955292

\section{Rights / license:}

In Copyright - Non-Commercial Use Permitted

\section{Originally published in:}

ETH, Eidgenössische Technische Hochschule Zürich, Departement Informatik, Institut für Wissenschaftliches Rechnen 217 
Eidgenössische

Technische Hochschule Zürich
Departement Informatik Institut für

Wissenschaftliches Rechnen
Walter Gander

Gene H. Golub

Rolf Strebel
Fitting of Circles and Ellipses

Least Squares Solution

June 1994 
ETH Zürich

Departement Informatik

Institut für Wissenschaftliches Rechnen

Prof. Dr. W. Gander

Walter Gander

Institut für Wissenschaftliches Rechnen

Eidgenössische Technische Hochschule

$\mathrm{CH}-8092$ Zürich

e-mail: gander@inf.ethz.ch

Gene H. Golub

Computer Science Department

Stanford University

Stanford, California 94305

e-mail: golub@sccm.stanford.edu

Rolf Strebel

Institut für Wissenschaftliches Rechnen

Eidgenössische Technische Hochschule

$\mathrm{CH}-8092$ Zürich

e-mail: strebel@inf.ethz.ch

This report is also available via anonymous ftp from ftp.inf.ethz.ch as doc/tech-reports/1994/217.ps.

(c) 1994 Departement Informatik, ETH Zürich 


\title{
Fitting of Circles and Ellipses Least Squares Solution
}

\author{
Walter Gander \\ Institut für Wissenschaftliches Rechnen \\ Eidgenössische Technische Hochschule \\ CH-8092 Zürich \\ gander@inf.ethz.ch
}

\author{
Gene H. Golub \\ Computer Science Department \\ Stanford University \\ Stanford, California 94305 \\ golubesccm.stanford . edu
}

\author{
Rolf Strebel \\ Institut für Wissenschaftliches Rechnen \\ Eidgenössische Technische Hochschule \\ CH-8092 Zürich \\ strebel@inf.ethz.ch
}

November 11, 1994

\begin{abstract}
Fitting circles and ellipses to given points in the plane is a problem that arises in many application areas, e.g. computer graphics [1], coordinate metrology [2], petroleum engineering [11], statistics [7]. In the past, algorithms have been given which fit circles and ellipses in some least squares sense without minimizing the geometric distance to the given points [1], [6].

In this paper ${ }^{1}$ we present several algorithms which compute the ellipse for which the sum of the squares of the distances to the given points is minimal. These algorithms are compared with classical simple and iterative methods.

Circles and ellipses may be represented algebraically i.e. by an equation of the form $F(\mathbf{x})=0$. If a point is on the curve then its coordinates $\mathbf{x}$ are a zero of the function $F$. Alternatively, curves may be represented in parametric form, which is well suited for minimizing the sum of the squares of the distances.
\end{abstract}

Keywords. least squares, curve fitting, singular value decomposition.

\footnotetext{
${ }^{1}$ This report and the MATLAB sources are available via anonymous ftp from ftp.inf.ethz.ch as doc/tech-reports/1994/217.ps and doc/tech-reports/1994/217. matlab.tar respectively. A shortened version of this report is to appear in BIT 34(1994), pp. 556-577.
} 


\section{Contents}

1 Preliminaries 5

2 Circle: Minimizing the algebraic distance 5

3 Circle: Minimizing the geometric distance 6

4 Circle: Geometric fit in parametric form 7

5 Ellipse: Minimizing the algebraic distance 9

6 Ellipse: Geometric fit in parametric form $\quad 12$

7 Ellipse: Iterative algebraic solutions $\quad 15$

7.1 Curvature weights . . . . . . . . . . . . . 15

7.2 Geometric distance weighting . . . . . . . . . . . 15

7.3 Circle weight algorithm $\ldots \ldots \ldots \ldots \ldots \ldots$

8 Comparison of geometric algorithms 21

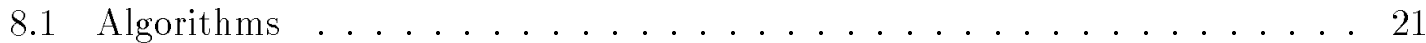

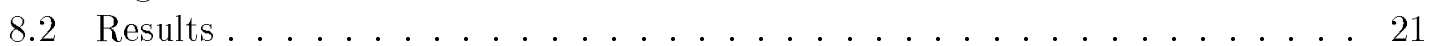

A Algorithms $\quad \mathbf{2 5}$

A.1 Gauss-Newton with Marquardt correction ............. 25

A.2 The Newton algorithm . . . . . . . . . . . . . 25

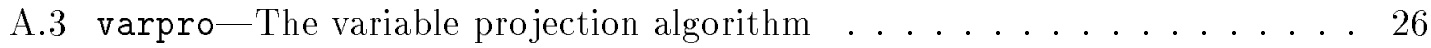

A.4 odr-The Orthogonal Distance Regression algorithm . . . . . . . . 27

B Matla Implementation $\quad \mathbf{2 7}$

B.1 Utilities . . . . . . . . . . . . . . . . . . 28

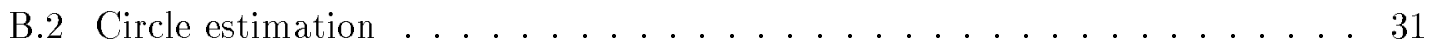

B.3 Algebraic ellipse estimation . . . . . . . . . . . . 33

B.4 Geometric ellipse estimation . . . . . . . . . . . . 38

B.4.1 Utilities . . . . . . . . . . . . . . . . 38

B.4.2 Gauss-Newton, Newton and Marquardt algorithms . . . . . . . . 40

B.4.3 The varpro algorithm . . . . . . . . . . . . . . 44

B.4.4 The odr algorithm ...................... 49

C Data sets $\quad \mathbf{5 5}$

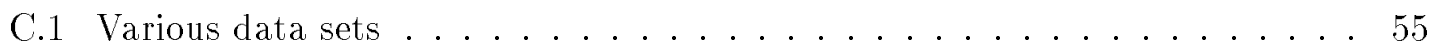

C.2 Comparison of the geometric estimation algorithms . . . . . . . 56 


\section{$1 \quad$ Preliminaries}

Ellipses, for which the sum of the squares of the distances to the given points is minimal will be referred to as "best fit" or "geometric fit", and the algorithms will be called "geometric".

Determining the parameters of the algebraic equation $F(\mathrm{x})=0$ in the least squares sense will be denoted by "algebraic fit" and the algorithms will be called "algebraic".

We will use the well known Gauss-Newton method to solve the nonlinear least squares problem [15]. Let $\mathbf{u}=\left(u_{1}, \ldots, u_{n}\right)^{\mathrm{T}}$ be a vector of unknowns and consider the nonlinear system of $m$ equations $\mathbf{f}(\mathbf{u})=\mathbf{0}$.

If $m>n$, then we want to minimize

$$
\sum_{i=1}^{m} f_{i}(\mathbf{u})^{2}=\min .
$$

This is a nonlinear least squares problem, which we will solve iteratively by a sequence of linear least squares problems.

We approximate the solution $\hat{\mathbf{u}}$ by $\tilde{\mathbf{u}}+\mathbf{h}$. Developing

$$
\mathbf{f}(\mathbf{u})=\left(f_{1}(\mathbf{u}), f_{2}(\mathbf{u}), \ldots, f_{m}(\mathbf{u})\right)^{\mathrm{T}}
$$

around $\tilde{\mathbf{u}}$ in a Taylor series, we obtain

$$
\mathbf{f}(\tilde{\mathbf{u}}+\mathbf{h}) \simeq \mathbf{f}(\tilde{\mathbf{u}})+J(\tilde{\mathbf{u}}) \mathbf{h} \approx \mathbf{0},
$$

where $J$ is the Jacobian. We solve equation (1) as a linear least squares problem for the correction vector $\mathbf{h}$ :

$$
J(\tilde{\mathbf{u}}) \mathbf{h} \approx-\mathbf{f}(\tilde{\mathbf{u}}) .
$$

An iteration then with the Gauss-Newton method consists of the two steps:

1. Solving equation (2) for $\mathbf{h}$.

2. Update the approximation $\tilde{\mathbf{u}}:=\tilde{\mathbf{u}}+\mathbf{h}$.

We define the following notation: a given point $P_{i}$ will have the coordinate vector $\mathbf{x}_{i}=\left(x_{i 1}, x_{i 2}\right)^{\mathrm{T}}$. The $m \times 2$ matrix $X=\left[\mathbf{x}_{1}, \ldots, \mathbf{x}_{m}\right]^{\mathrm{T}}$ will therefore contain the coordinates of a set of $m$ points. The 2 -norm $\|\cdot\|_{2}$ of vectors and matrices will simply be denoted by $\|\cdot\|$.

To improve the readability of the paper, we have moved the MatlaB implementation of the algorithms and some input data to the appendix. The MatlaB sources for the examples are available via anonymous ftp from ftp.inf.ethz.ch.

\section{Circle: Minimizing the algebraic distance}

Let us first consider an algebraic representation of the circle in the plane:

$$
F(\mathbf{x})=a \mathbf{x}^{\mathrm{T}} \mathbf{x}+\mathbf{b}^{\mathrm{T}} \mathbf{x}+c=0,
$$

where $a \neq 0$ and $\mathbf{x}, \mathbf{b} \in \mathbb{R}^{2}$. To fit a circle, we need to compute the coefficients $a, \mathbf{b}$ and $c$ from the given data points. 
If we insert the coordinates of the points into equation (3), we obtain a linear system of equations $B \mathbf{u}=\mathbf{0}$ for the coefficients $\mathbf{u}=\left(a, b_{1}, b_{2}, c\right)^{\mathrm{T}}$, where

$$
B=\left(\begin{array}{cccc}
x_{11}^{2}+x_{12}^{2} & x_{11} & x_{12} & 1 \\
\vdots & \vdots & \vdots & \vdots \\
x_{m 1}^{2}+x_{m 2}^{2} & x_{m 1} & x_{m 2} & 1
\end{array}\right)
$$

To obtain a non-trivial solution, we impose some constraint on $\mathbf{u}$, e.g. $u_{1}=1$ (commonly used) or $\|\mathbf{u}\|=1$.

For $m>3$, in general, we cannot expect the system to have a solution, unless all the points happen to be on a circle. Therefore, we solve the overdetermined system $B \mathbf{u}=\mathbf{r}$ where $\mathbf{u}$ is chosen to minimize $\|\mathbf{r}\|$. We obtain a standard problem (c.f. [3]):

$$
\|B \mathbf{u}\|=\min \quad \text { subject to } \quad\|\mathbf{u}\|=1 .
$$

This problem is equivalent to finding the right singular vector associated with the smallest singular value of $B$. If $a \neq 0$, we can transform equation (3) to

$$
\left(x_{1}+\frac{b_{1}}{2 a}\right)^{2}+\left(x_{2}+\frac{b_{2}}{2 a}\right)^{2}=\frac{\|\mathbf{b}\|^{2}}{4 a^{2}}-\frac{c}{a},
$$

from which we obtain the center and the radius, if the right hand side of (4) is positive:

$$
\mathbf{z}=\left(z_{1}, z_{2}\right)=\left(-\frac{b_{1}}{2 a},-\frac{b_{2}}{2 a}\right) \quad r=\sqrt{\frac{\|\mathbf{b}\|^{2}}{4 a^{2}}-\frac{c}{a}} .
$$

The MatlaB procedure algcircle computes the center $\mathbf{z}=\left(z_{1}, z_{2}\right)$ and the radius $r$ of the circle by minimizing the algebraic distance. This approach has the advantage of being simple. The disadvantage is that we are uncertain what we are minimizing in a geometrical sense. For applications in coordinate metrology this kind of fit is often unsatisfactory. In such applications, one wishes to minimize the sum of the squares of the distances. Figure 1 shows two circles fitted to the set of points

$$
\begin{array}{l|llllll}
x & 1 & 2 & 5 & 7 & 9 & 3 \\
\hline y & 7 & 6 & 8 & 7 & 5 & 7
\end{array} .
$$

Minimizing the algebraic distance, we obtain the dashed circle with radius $r=3.0370$ and center $\mathbf{z}=(5.3794,7.2532)$.

The algebraic solution is often useful as a starting vector for methods minimizing the geometric distance.

\section{Circle: Minimizing the geometric distance}

To minimize the sum of the squares of the distances $d_{i}^{2}=\left(\left\|\mathbf{z}-\mathbf{x}_{i}\right\|-r\right)^{2}$ we need to solve a nonlinear least squares problem. Let $\mathbf{u}=\left(z_{1}, z_{2}, r\right)^{\mathrm{T}}$, we want to determine $\tilde{\mathbf{u}}$ so that

$$
\sum_{i=1}^{m} d_{i}(\mathbf{u})^{2}=\min
$$




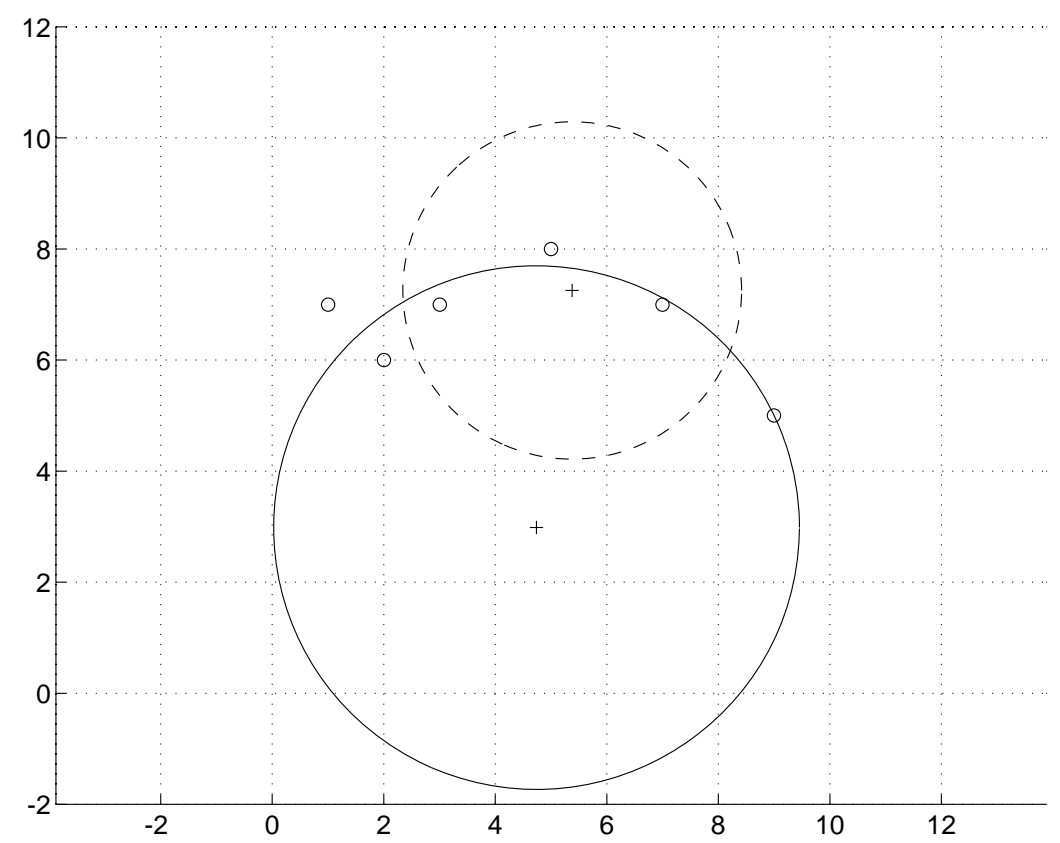

Figure 1: algebraic vs. best fit

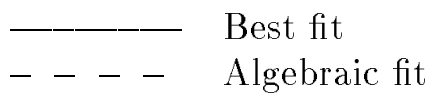

The Jacobian defined by the partial derivatives $\partial d_{i}(\mathbf{u}) / \partial u_{j}$ is given by:

$$
J(\mathbf{u})=\left(\begin{array}{ccc}
\frac{u_{1}-x_{11}}{\sqrt{\left(u_{1}-x_{11}\right)^{2}+\left(u_{2}-x_{12}\right)^{2}}} & \frac{u_{2}-x_{12}}{\sqrt{\left(u_{1}-x_{11}\right)^{2}+\left(u_{2}-x_{12}\right)^{2}}} & -1 \\
\vdots & \frac{u_{2}-x_{2 m}}{u_{1}-x_{m 1}} & \vdots \\
\frac{\sqrt{\left(u_{1}-x_{m 1}\right)^{2}+\left(u_{2}-x_{m 2}\right)^{2}}}{\sqrt{\left(u_{1}-x_{m 1}\right)^{2}+\left(u_{2}-x_{m 2}\right)^{2}}} & -1
\end{array}\right) .
$$

A good starting vector for the Gauss-Newton method may often be obtained by solving the linear problem as given in the previous paragraph. The MatLAB procedure circle then iteratively computes the "best" circle.

If we use the set of points (5) and start the iteration with the values obtained from the linear model (minimizing the algebraic distance), then after 11 Gauss-Newton steps the norm of the correction vector is $2.05 E-6$. We obtain the best fit circle with center $\mathbf{z}=(4.7398,2.9835)$ and radius $r=4.7142$ (the solid circle in figure 1 ).

\section{Circle: Geometric fit in parametric form}

The parametric form commonly used for the circle is given by

$$
\begin{aligned}
& x=z_{1}+r \cos \varphi \\
& y=z_{2}+r \sin \varphi .
\end{aligned}
$$


The distance $d_{i}$ of a point $P_{i}=\left(x_{i 1}, x_{i 2}\right)$ may be expressed by

$$
d_{i}^{2}=\min _{\varphi_{i}}\left[\left(x_{i 1}-x\left(\varphi_{i}\right)\right)^{2}+\left(x_{i 2}-y\left(\varphi_{i}\right)\right)^{2}\right] .
$$

Now since we want to determine $z_{1}, z_{2}$ and $r$ by minimizing

$$
\sum_{i=1}^{m} d_{i}^{2}=\min
$$

we can simultaneously minimize for $z_{1}, z_{2}, r$ and $\left\{\varphi_{i}\right\}_{i=1 \ldots m}$; i.e. find the minimum of the quadratic function

$$
Q\left(\varphi_{1}, \varphi_{2}, \ldots, \varphi_{m}, z_{1}, z_{2}, r\right)=\sum_{i=1}^{m}\left[\left(x_{i 1}-x\left(\varphi_{i}\right)\right)^{2}+\left(x_{i 2}-y\left(\varphi_{i}\right)\right)^{2}\right]
$$

This is equivalent to solving the nonlinear least squares problem

$$
\begin{aligned}
& z_{1}+r \cos \varphi_{i}-x_{i 1} \approx 0 \\
& z_{2}+r \sin \varphi_{i}-x_{i 2} \approx 0 \quad \text { for } i=1,2, \ldots, m .
\end{aligned}
$$

Let $\mathbf{u}=\left(\varphi_{1}, \ldots, \varphi_{m}, z_{1}, z_{2}, r\right)$. The Jacobian associated with $Q$ is

$$
J=\left(\begin{array}{rr}
r S & A \\
-r C & B
\end{array}\right)
$$

where $S=\operatorname{diag}\left(\sin \varphi_{i}\right)$ and $C=\operatorname{diag}\left(\cos \varphi_{i}\right)$ are $m \times m$ diagonal matrices. $A$ and $B$ are $m \times 3$ matrices defined by:

$$
\begin{aligned}
& a_{i 1}=-1 \quad a_{i 2}=0 \quad a_{i 3}=-\cos \varphi_{i} \\
& b_{i 1}=0 \quad b_{i 2}=-1 \quad b_{i 3}=-\sin \varphi_{i} \text {. }
\end{aligned}
$$

For large $m, J$ is very sparse. We note that the first part $\left(\begin{array}{c}r S \\ -r C\end{array}\right)$ is orthogonal. To compute the $Q R$ decomposition of $J$ we use the orthonormal matrix

$$
Q=\left(\begin{array}{cc}
S & C \\
-C & S
\end{array}\right)
$$

Multiplying from the left we get

$$
Q^{\mathrm{T}} J=\left(\begin{array}{cc}
r I & S A-C B \\
O & C A+S B
\end{array}\right)
$$

So to obtain the $Q R$ decomposition of the Jacobian, we only have to compute a $Q R$ decomposition of the $m \times 3$ sub-matrix $C A+S B=U P$. Then

$$
\left(\begin{array}{cc}
I & 0 \\
O & U^{\mathrm{T}}
\end{array}\right) Q^{\mathrm{T}} J=\left(\begin{array}{cc}
r I & S A-C B \\
O & P
\end{array}\right)
$$

and the solution is obtained by backsubstitution. In general we may obtain good starting values for $z_{1}, z_{2}$ and $r$ for the Gauss-Newton iteration, if we first solve the linear problem 
by minimizing the algebraic distance. If the center is known, initial approximations for $\left\{\varphi_{k}\right\}_{k=1 \ldots m}$ can be computed by

$$
\varphi_{k}=\arg \left(\left(x_{k 1}-z_{1}\right)+i\left(x_{k 2}-z_{2}\right)\right) .
$$

The Matlab procedure parcircle computes the best fit circle using the parametric form. We use again the points (5) and start the iteration with the values obtained from the linear model (minimizing the algebraic distance). After 21 Gauss-Newton steps the norm of the correction is $3.43 E-06$ and we obtain the same results as before: center $\mathbf{z}=(4.7398,2.9835)$ and radius $r=4.7142$ (the solid circle in figure 1 ).

\section{Ellipse: Minimizing the algebraic distance}

Given the quadratic equation

$$
\mathrm{x}^{\mathrm{T}} A \mathrm{x}+\mathbf{b}^{\mathrm{T}} \mathrm{x}+c=0
$$

with $A$ symmetric and positive definite, we can compute the geometric quantities of the conic as follows.

We introduce new coordinates $\overline{\mathbf{x}}$ with $\mathbf{x}=Q \overline{\mathbf{x}}+\mathbf{t}$, thus rotating and shifting the conic. Then equation (8) becomes

$$
\overline{\mathbf{x}}^{\mathrm{T}}\left(Q^{\mathrm{T}} A Q\right) \overline{\mathbf{x}}+\left(2 \mathbf{t}^{\mathrm{T}} A+\mathbf{b}^{\mathrm{T}}\right) Q \overline{\mathbf{x}}+\mathbf{t}^{\mathrm{T}} A \mathbf{t}+\mathbf{b}^{\mathrm{T}} \mathbf{t}+c=0 .
$$

Defining $\bar{A}=Q^{\mathrm{T}} A Q$, and similarly $\overline{\mathbf{b}}$ and $\bar{c}$, this equation may be written

$$
\overline{\mathbf{x}}^{\mathrm{T}} \bar{A} \overline{\mathbf{x}}+\overline{\mathbf{b}}^{\mathrm{T}} \overline{\mathbf{x}}+\bar{c}=0 .
$$

We may choose $Q$ so that $\bar{A}=\operatorname{diag}\left(\lambda_{1}, \lambda_{2}\right)$; if the conic is an ellipse or a hyperbola, we may further choose $\mathbf{t}$ so that $\overline{\mathbf{b}}=\mathbf{0}$. Hence, the equation may be written

$$
\lambda_{1} \bar{x}_{1}^{2}+\lambda_{2} \bar{x}_{2}^{2}+\bar{c}=0
$$

and this defines an ellipse if $\lambda_{1}>0, \lambda_{2}>0$ and $\bar{c}<0$. The center and the axes of the ellipse in the non-transformed system are given by

$$
\begin{aligned}
\mathrm{z} & =\mathrm{t} \\
a & =\sqrt{-\bar{c} / \lambda_{1}} \\
b & =\sqrt{-\bar{c} / \lambda_{2}} .
\end{aligned}
$$

Since $Q^{\mathrm{T}} Q=I$, the matrices $A$ and $\bar{A}$ have the same (real) eigenvalues $\lambda_{1}, \lambda_{2}$. It follows that each function of $\lambda_{1}$ and $\lambda_{2}$ is invariant under rotation and shifts. Note

$$
\begin{aligned}
\operatorname{det} A & =a_{11} a_{22}-a_{21} a_{12}=\lambda_{1} \lambda_{2} \\
\operatorname{trace} A & =a_{11}+a_{22}=\lambda_{1}+\lambda_{2},
\end{aligned}
$$

which serve as a basis for all polynomials symmetric in $\lambda_{1}, \lambda_{2}$. As a possible application of above observations, let us express the quotient $\kappa=a / b$ for the ellipse's axes $a$ and $b$. With $a^{2}=-\bar{c} / \lambda_{1}$ and $b^{2}=-\bar{c} / \lambda_{2}$ we get

$$
\kappa^{2}+1 / \kappa^{2}=\frac{\lambda_{2}}{\lambda_{1}}+\frac{\lambda_{1}}{\lambda_{2}}=\frac{\lambda_{1}^{2}+\lambda_{2}^{2}}{\lambda_{1} \lambda_{2}}=\frac{(\operatorname{trace} A)^{2}-2 \operatorname{det} A}{\operatorname{det} A}=\frac{a_{11}^{2}+a_{22}^{2}+2 a_{12}^{2}}{a_{11} a_{22}-a_{12}^{2}}
$$


and therefore

where

$$
\kappa^{2}=\mu \pm \sqrt{\mu^{2}-1}
$$

$$
\mu=\frac{(\operatorname{trace} A)^{2}}{2 \operatorname{det} A}-1
$$

To compute the coefficients $\mathbf{u}$ from given points, we insert the coordinates into equation (8) and obtain a linear system of equations $B \mathbf{u}=0$, which we may solve again as constrained least squares problem: $\|B \mathbf{u}\|=\min$ subject to $\|\mathbf{u}\|=1$. The MatLaB procedure algellipse fits an ellipse by minimizing this algebraic distance.

The disadvantage of the constraint $\|\mathbf{u}\|=1$ is its non-invariance for Euclidean coordinate transformations

$$
\mathbf{x}=Q \overline{\mathbf{x}}+\mathbf{t}, \quad \text { where } Q^{\mathrm{T}} Q=I .
$$

For this reason Bookstein [9] recommended solving the constrained least squares problem

$$
\begin{aligned}
\mathbf{x}^{\mathrm{T}} A \mathbf{x}+\mathbf{b}^{\mathrm{T}} \mathbf{x}+c & \approx 0 \\
\lambda_{1}^{2}+\lambda_{2}^{2}=a_{11}^{2}+2 a_{12}^{2}+a_{22}^{2} & =1 .
\end{aligned}
$$

While [9] describes a solution based on eigenvalue decomposition, we may solve the same problem more efficiently and accurately with a singular value decomposition as described in [12]. In the simple algebraic solution by SVD, we solve the system for the parameter vector

$$
\mathbf{u}=\left(a_{11}, 2 a_{12}, a_{22}, b_{1}, b_{2}, c\right)^{\mathrm{T}}
$$

with the constraint $\|\mathbf{u}\|=1$, which is not invariant under Euclidean transformations. If we define vectors

$$
\begin{aligned}
\mathbf{v} & =\left(b_{1}, b_{2}, c\right)^{\mathrm{T}} \\
\mathbf{w} & =\left(a_{11}, \sqrt{2} a_{12}, a_{22}\right)^{\mathrm{T}}
\end{aligned}
$$

and the coefficient matrix

$$
S=\left(\begin{array}{cccccc}
x_{11} & x_{12} & 1 & x_{11}^{2} & \sqrt{2} x_{11} x_{12} & x_{12}^{2} \\
\vdots & \vdots & \vdots & \vdots & \vdots & \vdots \\
x_{m 1} & x_{m 2} & 1 & x_{m 1}^{2} & \sqrt{2} x_{m 1} x_{m 2} & x_{m 2}^{2}
\end{array}\right),
$$

then the Bookstein constraint (11) may be written $\|\mathbf{w}\|=1$, and we have the reordered system

$$
S\left(\begin{array}{c}
\mathrm{v} \\
\mathrm{w}
\end{array}\right) \approx 0
$$

The $Q R$ decomposition of $S$ leads to the equivalent system

$$
\left(\begin{array}{cc}
R_{11} & R_{12} \\
0 & R_{22}
\end{array}\right)\left(\begin{array}{c}
\mathbf{v} \\
\mathbf{w}
\end{array}\right) \approx \mathbf{0}
$$

which may be solved in following steps:

$$
\begin{aligned}
R_{22} \mathbf{w} & \approx \mathbf{0} \\
\|\mathbf{w}\| & =1 .
\end{aligned}
$$


Using the singular value decomposition of $R_{22}=U \Sigma V^{\mathrm{T}}$, finding $\mathbf{w}=\mathbf{v}_{3}$, and then

$$
\mathbf{v}=-R_{11}^{-1} R_{12} \mathbf{w}
$$

The MATLAB procedure bookstein and the MATLAB procedure bookstein_svd implement the two algorithms. Note that the problem

$$
\left(S_{1} S_{2}\right)\left(\begin{array}{c}
\mathbf{v} \\
\mathbf{w}
\end{array}\right) \approx \mathbf{0} \quad \text { where }\|\mathbf{w}\|=1
$$

is equivalent to the generalized total least squares problem finding a matrix $\hat{S}_{2}$ such that

$$
\begin{aligned}
\operatorname{rank}\left(S_{1} \hat{S_{2}}\right) & \leq 5 \\
\left\|\left(S_{1} \hat{S_{2}}\right)-\left(S_{1} S_{2}\right)\right\| & =\inf _{\operatorname{rank}\left(S_{1} \bar{S}_{2}\right) \leq 5}\left\|\left(S_{1} \overline{S_{2}}\right)-\left(S_{1} S_{2}\right)\right\| .
\end{aligned}
$$

In other words, find a best rank 5 approximation to $S$ that leaves $S_{1}$ fixed. A description of this problem may be found in [16].

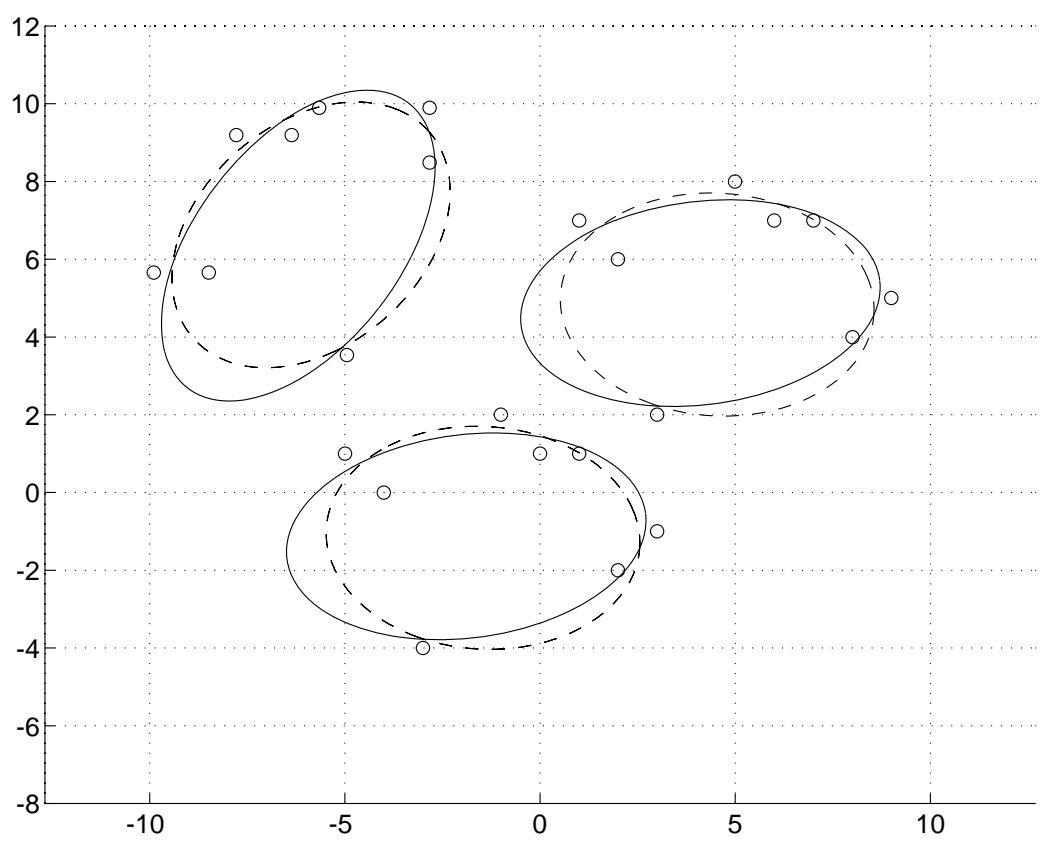

Figure 2: Euclidean-invariant algorithms

$$
\begin{array}{ll}
- \text { Constraint } \lambda_{1}^{2}+\lambda_{2}^{2}=1 \\
----\quad \text { Constraint } \lambda_{1}+\lambda_{2}=1
\end{array}
$$

To demonstrate the influence of different coordinate systems, we have computed the ellipse fit for this set of points:

$$
\begin{array}{l|llllllll}
x & 1 & 2 & 5 & 7 & 9 & 6 & 3 & 8 \\
\hline y & 7 & 6 & 8 & 7 & 5 & 7 & 2 & 4
\end{array},
$$




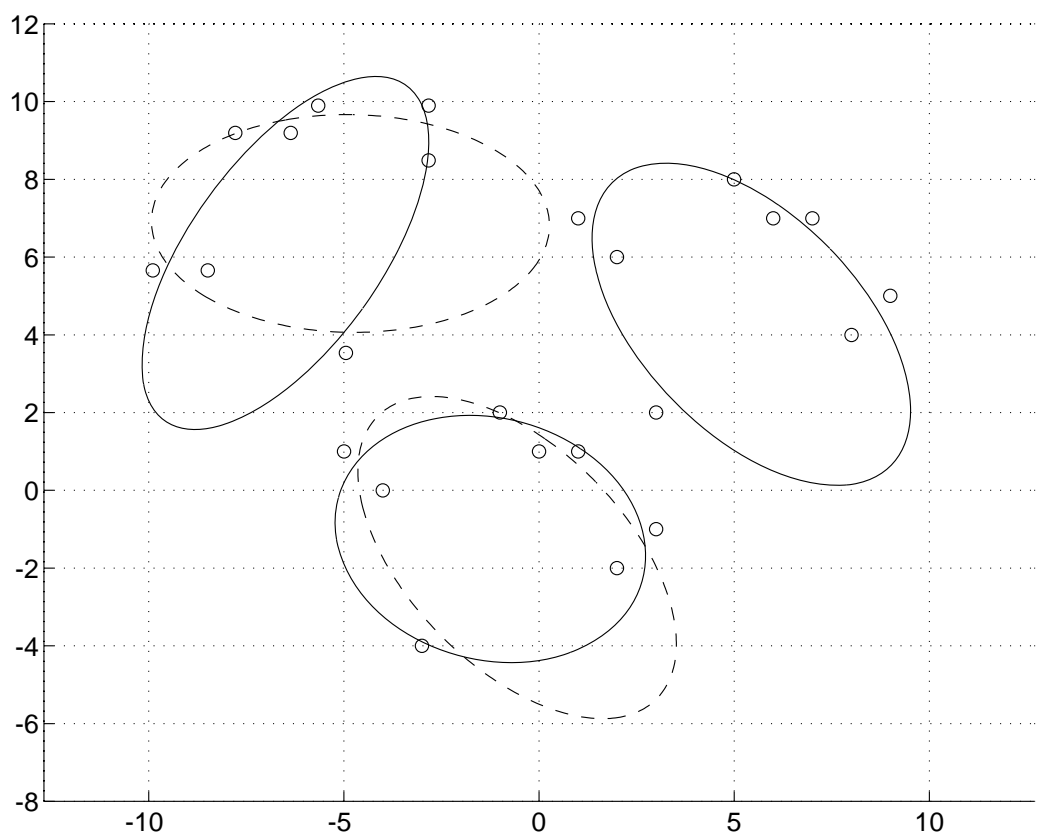

Figure 3: Non-invariant algebraic algorithm

Fitted ellipses

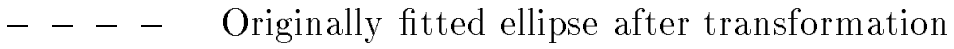

which are first shifted by $(-6,-6)$, and then by $(-4,4)$ and rotated by $\pi / 4$. See figures $2-3$ for the fitted ellipses.

Since $\lambda_{1}^{2}+\lambda_{2}^{2} \neq 0$ for ellipses, hyperbolas and parabolas, the Bookstein constraint is appropriate to fit any of these. But all we need is an invariant $I \neq 0$ for ellipses-and one of them is $\lambda_{1}+\lambda_{2}$. Thus we may invariantly fit an ellipse with the constraint

$$
\lambda_{1}+\lambda_{2}=a_{11}+a_{22}=1
$$

which results in the linear least squares problem

$$
\left(\begin{array}{ccccc}
2 x_{11} x_{12} & x_{22}^{2}-x_{11}^{2} & x_{11} & x_{12} & 1 \\
\vdots & \vdots & & & \vdots \\
2 x_{m 1} x_{m 2} & x_{m 2}^{2}-x_{m 1}^{2} & x_{m 1} & x_{m 2} & 1
\end{array}\right) \mathbf{v} \approx\left(\begin{array}{c}
-x_{11}^{2} \\
\vdots \\
-x_{m 1}^{2}
\end{array}\right)
$$

See the dashed ellipses in figure 2. The MaTLAB procedure alge_simple implements this algorithm.

\section{Ellipse: Geometric fit in parametric form}

In order to fit an ellipse in parametric form, we consider the equations

$$
\mathrm{x}=\mathrm{z}+Q(\alpha) \mathrm{x}^{\prime}, \quad \mathrm{x}^{\prime}=\left(\begin{array}{c}
a \cos \varphi \\
b \sin \varphi
\end{array}\right), \quad Q(\alpha)=\left(\begin{array}{cc}
\cos \alpha & -\sin \alpha \\
\sin \alpha & \cos \alpha
\end{array}\right) .
$$


Minimizing the sum of squares of the distances of the given points to the "best" ellipse is equivalent to solving the nonlinear least squares problem:

$$
\mathbf{g}_{i}=\left(\begin{array}{l}
x_{i 1} \\
x_{i 2}
\end{array}\right)-\left(\begin{array}{l}
z_{1} \\
z_{2}
\end{array}\right)-Q(\alpha)\left(\begin{array}{c}
a \cos \varphi_{i} \\
b \sin \varphi_{i}
\end{array}\right) \approx 0, \quad i=1, \ldots, m .
$$

Thus we have $2 m$ nonlinear equations for $m+5$ unknowns: $\varphi_{1}, \ldots, \varphi_{m}, \alpha, a, b, z_{1}, z_{2}$. To compute the Jacobian we need the partial derivatives:

$$
\begin{aligned}
\frac{\partial \mathbf{g}_{i}}{\partial \varphi_{j}} & =-\delta_{i j} Q(\alpha)\left(\begin{array}{c}
-a \sin \varphi_{i} \\
b \cos \varphi_{i}
\end{array}\right) \\
\frac{\partial \mathbf{g}_{i}}{\partial \alpha} & =-\dot{Q}(\alpha)\left(\begin{array}{c}
a \cos \varphi_{i} \\
b \sin \varphi_{i}
\end{array}\right) \\
\frac{\partial \mathbf{g}_{i}}{\partial a} & =-Q(\alpha)\left(\begin{array}{c}
\cos \varphi_{i} \\
0
\end{array}\right) \\
\frac{\partial \mathbf{g}_{i}}{\partial b} & =-Q(\alpha)\left(\begin{array}{c}
0 \\
\sin \varphi_{i}
\end{array}\right) \\
\frac{\partial \mathbf{g}_{i}}{\partial z_{1}} & =\left(\begin{array}{c}
-1 \\
0
\end{array}\right) \\
\frac{\partial \mathbf{g}_{i}}{\partial z_{2}} & =\left(\begin{array}{c}
0 \\
-1
\end{array}\right)
\end{aligned}
$$

where we have used the notation

$$
\delta_{i j}=\left\{\begin{array}{ll}
1, & i=j \\
0, & i \neq j
\end{array} .\right.
$$

Thus the Jacobian becomes:

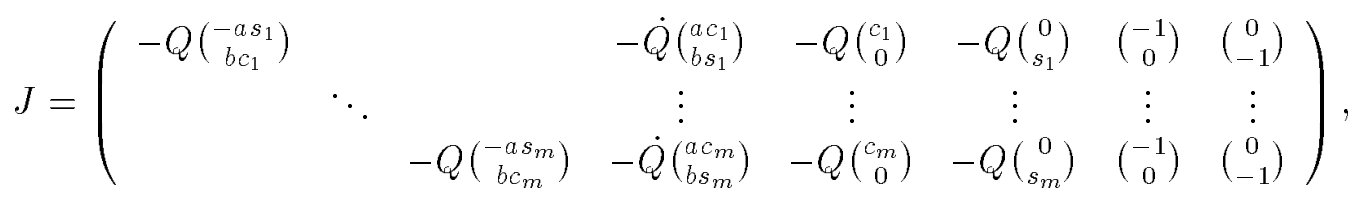

where we have used as abbreviation $s_{i}=\sin \varphi_{i}$ and $c_{i}=\cos \varphi_{i}$. Note that

$$
\dot{Q}(\alpha)=\left(\begin{array}{cc}
-\sin \alpha & -\cos \alpha \\
\cos \alpha & -\sin \alpha
\end{array}\right) \text { and therefore } Q^{\mathrm{T}} \dot{Q}=\left(\begin{array}{cc}
0 & -1 \\
1 & 0
\end{array}\right) .
$$

Since $Q$ is orthogonal, the $2 m \times 2 m$ block diagonal matrix $U=-\operatorname{diag}(Q, \ldots, Q)$ is orthogonal, too, and

$$
U^{\mathrm{T}} J=\left(\begin{array}{cccccccc}
\left(\begin{array}{c}
-a s_{1} \\
b c_{1}
\end{array}\right) & & & \left(\begin{array}{c}
-b s_{1} \\
a c_{1}
\end{array}\right) & \left(\begin{array}{c}
c_{1} \\
0
\end{array}\right) & \left(\begin{array}{c}
0 \\
s_{1}
\end{array}\right) & \left(\begin{array}{c}
c \\
-s
\end{array}\right) & \left(\begin{array}{c}
s \\
c
\end{array}\right) \\
& \ddots & & \vdots & \vdots & \vdots & \vdots & \vdots \\
& & \left(\begin{array}{c}
-a s_{m} \\
b c_{m}
\end{array}\right) & \left(\begin{array}{c}
-b s_{m} \\
a c_{m}
\end{array}\right) & \left(\begin{array}{c}
c_{m} \\
0
\end{array}\right) & \left(\begin{array}{c}
0 \\
s_{m}
\end{array}\right) & \left(\begin{array}{c}
c \\
-s
\end{array}\right) & \left(\begin{array}{c}
s \\
c
\end{array}\right)
\end{array}\right),
$$

where $s=\sin \alpha$ and $c=\cos \alpha$. If we permute the equations, we obtain a similar structure for the Jacobian as in the circle fit:

$$
\bar{J}=\left(\begin{array}{rr}
-a S & A \\
b C & B
\end{array}\right)
$$


That is, $S=\operatorname{diag}\left(\sin \varphi_{i}\right)$ and $C=\operatorname{diag}\left(\cos \varphi_{i}\right)$ are two $m \times m$ diagonal matrices and $A$ and $B$ are $m \times 5$ and are defined by:

$$
\begin{aligned}
& A(i, 1: 5)=\left[\begin{array}{ccccc}
-b \sin \varphi_{i} & \cos \varphi_{i} & 0 & \cos \alpha & \sin \alpha
\end{array}\right] \\
& B(i, 1: 5)=\left[\begin{array}{ccccc}
a \cos \varphi_{i} & 0 & \sin \varphi_{i} & -\sin \alpha & \cos \alpha
\end{array}\right]
\end{aligned}
$$

We cannot give an explicit expression for an orthogonal matrix to triangularize the first $m$ columns of $J$ in a similar way as we did in fitting a circle. However, we can use Givens rotations to do this in $m$ steps. The MATLAB procedure rot_cossin-which computes the angles for the Givens rotation matrices - is used by the MatLaB procedure pare to compute the best ellipse fit using the parametric form.

Figure 4 shows two ellipses fitted to the points given by

$$
\begin{array}{l|llllllll}
x & 1 & 2 & 5 & 7 & 9 & 3 & 6 & 8 \\
\hline y & 7 & 6 & 8 & 7 & 5 & 7 & 2 & 4
\end{array} .
$$

By minimizing the algebraic distance with $\|\mathbf{u}\|=1$ we obtain the large cigar shaped dashed ellipse with $\mathbf{z}=(13.8251,-2.1099), a=29.6437, b=1.8806$ and residual norm $r=1.80$. If we minimize the sum of squares of the distances then we obtain the solid ellipse with $\mathbf{z}=(2.6996,3.8160), a=6.5187, b=3.0319$ and $r=1.17$. In order to obtain

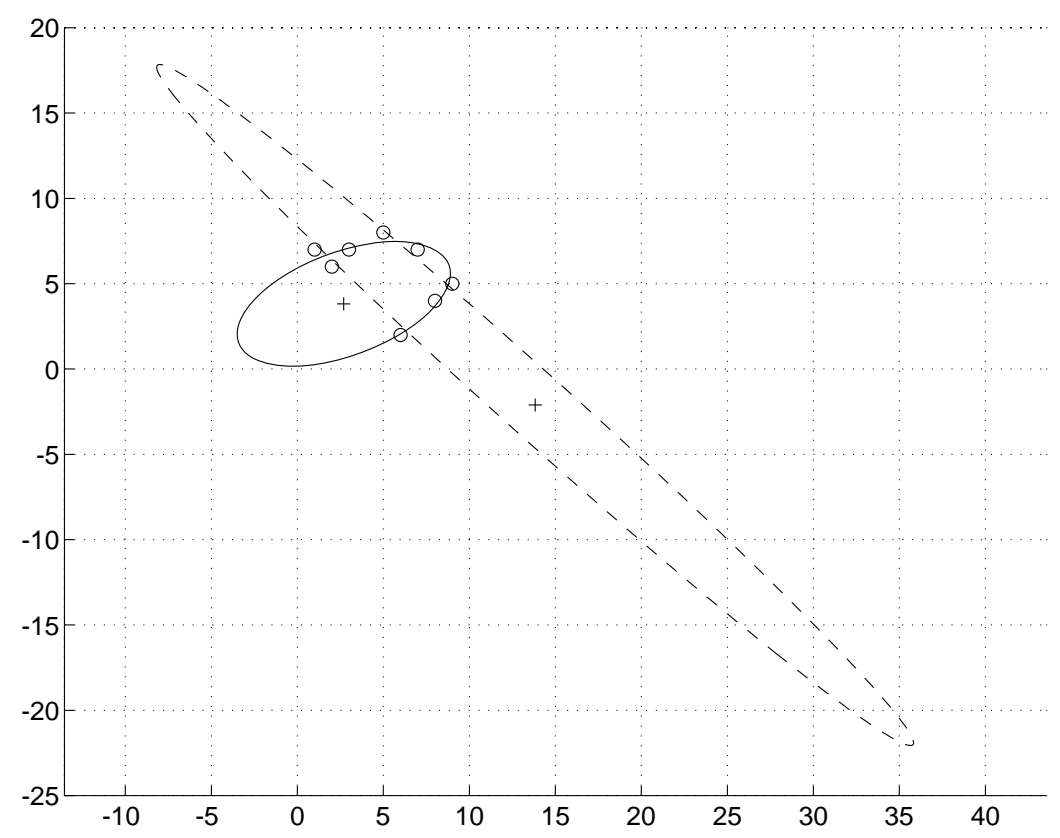

Figure 4: algebraic versus best fit

$$
\begin{array}{ll}
- \text { Best fit } \\
---- & \text { Algebraic fit }(\|\mathbf{u}\|=1)
\end{array}
$$

starting values for the nonlinear least squares problem we used the center, obtained by fitting the best circle. We cannot use the approximation $b_{0}=a_{0}=r$, since the Jacobian becomes singular for $b=a$ ! Therefore, we used $b_{0}=r / 2$ as a starting value. With $\alpha_{0}=0$, we needed 71 iteration steps to compute the "best ellipse" shown in Figure 4. 


\section{Ellipse: Iterative algebraic solutions}

In this section, we will present modifications to the algebraic fit of ellipses. The algebraic equations may be weighted depending on a given estimation-thus leading to a simple iterative mechanism. Most algorithms try to weight the points such that the algebraic solution comes closer to the geometric solution. Another idea is to favor non-eccentric ellipses.

\subsection{Curvature weights}

The solution of

$$
\mathbf{x}^{\mathrm{T}} A \mathbf{x}+\mathbf{b}^{\mathrm{T}} \mathrm{x}+c \approx 0
$$

in the least squares sense leads to an equation for each point. If the equation for point $\left(x_{i 1}, x_{i 2}\right)$ is multiplied by $\omega_{i}>1$, the solution will approximate this point more accurately. In [6], $\omega_{i}$ is set to $1 / R_{i}$, where $R_{i}$ is the curvature radius of the ellipse at a point $\mathbf{p}_{i}$ associated with $\left(x_{i 1}, x_{i 2}\right)$. The point $\mathbf{p}_{i}$ is determined by intersecting the ray from the ellipse's center to $\left(x_{i 1}, x_{i 2}\right)$ and the ellipse. The MATLAB procedure lyle fits ellipses using these curvature weights.

Tests on few data sets show, that this weighting scheme leads to better shaped ellipses in some cases, especially for eccentric ellipses; but it does not systematically restrict the solutions to ellipses. Lets look at the curvature weight solution for two problems. Figure 5 shows the result for the data set (14) presented earlier: unluckily, the algorithm finds a hyperbola for the weighted equations in the first step. On the other side, the algorithm is successful indeed for the data set in appendix C.1. Figure 6 shows the large solid ellipse (residual norm 2.20) found by the curvature weights algorithm. The small dotted ellipse is the solution of the unweighted algebraic solution (6.77); the dashed ellipse is the best fit solution using Gauss-Newton (1.66), and the dash-dotted ellipse (1.69) is found by the geometric-weight algorithm described later.

\subsection{Geometric distance weighting}

We are interested in weighting schemes which result in a least square solution for the geometric distance. If we define

$$
Q(\mathbf{x})=\mathbf{x}^{\mathrm{T}} A \mathbf{x}+\mathbf{b}^{\mathrm{T}} \mathbf{x}+c,
$$

then the simple algebraic method minimizes $Q$ for the given points in the least squares sense. $Q$ has the following geometric meaning: Let $h(\mathrm{x})$ be the geometric distance from the center to point $\mathrm{x}$

$$
h(\mathrm{x})=\sqrt{\left(x_{1}-z_{1}\right)^{2}+\left(x_{2}-z_{2}\right)^{2}}
$$

and determine $\mathbf{p}_{i}$ by intersecting the ray from the ellipse's center to $\mathbf{x}_{i}$ and the ellipse. Then, as pointed out in [9]

$$
\begin{aligned}
Q\left(\mathbf{x}_{i}\right) & =\kappa\left(\left(h\left(\mathbf{x}_{i}\right) / h\left(\mathbf{p}_{i}\right)\right)^{2}-1\right) \\
& \simeq 2 \kappa \frac{h\left(\mathbf{x}_{i}\right)-h\left(\mathbf{p}_{i}\right)}{h\left(\mathbf{p}_{i}\right)}, \quad \text { if } \mathbf{x}_{i} \simeq \mathbf{p}_{i}
\end{aligned}
$$

for some constant $\kappa$. This explains why the simple algebraic solution tends to neglect points far from the center. 


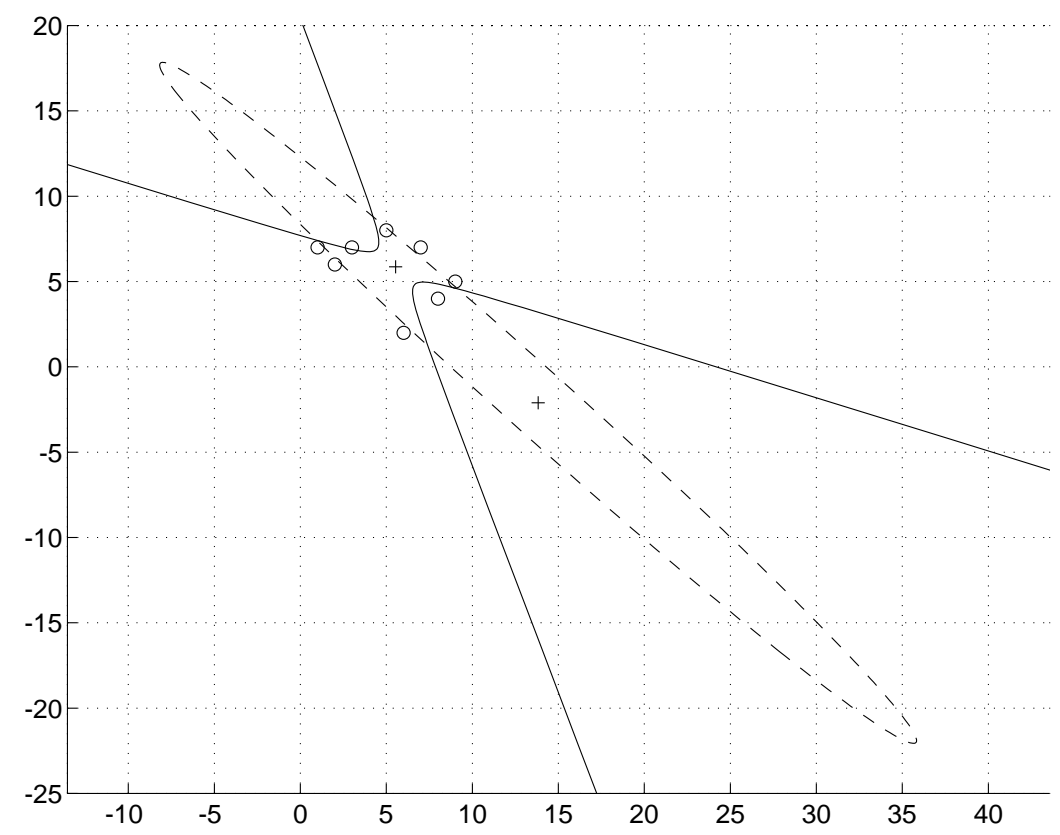

Figure 5: algebraic fit with curvature weights

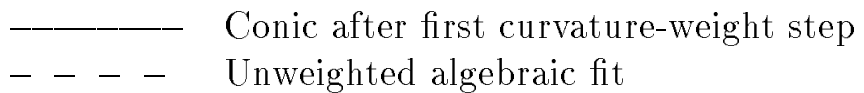

Thus, we may say that the algebraic solution fits the ellipse with respect to the relative distances, i.e. a distant point has not the same importance as a near point. If we prefer to minimize the absolute distances, we may solve a weighted problem with weights

$$
\omega_{i}=h\left(\mathbf{p}_{i}\right)
$$

for a given estimated ellipse. The resulting estimated ellipse may then be used to determine new weights $\omega_{i}$, thus iteratively solving weighted least squares problems.

Consequently, we may go a step further and set weights so that the equations are solved in the least squares sense for the geometric distances. If $d(\mathrm{x})$ is the geometric distance of $\mathrm{x}$ from the currently estimated ellipse, then weights are set

$$
\omega_{i}=d\left(\mathbf{x}_{i}\right) / Q\left(\mathbf{x}_{i}\right) \text {. }
$$

The Matlaв procedure wate 2 implements this geometric weight algorithm. See the dashdotted ellipse in figure 6 for an example.

The advantage of this method compared to the non-linear method to compute the geometric fit is, that no derivatives for the Jacobian or Hessian matrices are needed. The disadvantage of this method is, that it does not generally minimize the geometric distance. To show this, let us restate the problem:

$$
\|G(\mathrm{x}) \mathrm{x}\|^{2}=\min \quad \text { where }\|\mathrm{x}\|=1 .
$$

An iterative algorithm determines a sequence $\left(\mathbf{y}_{i}\right)$, where $\mathbf{y}_{k+1}$ is the solution of

$$
\left\|G\left(\mathbf{y}_{k}\right) \mathbf{y}\right\|^{2}=\min \quad \text { where }\|\mathbf{y}\|=1 .
$$




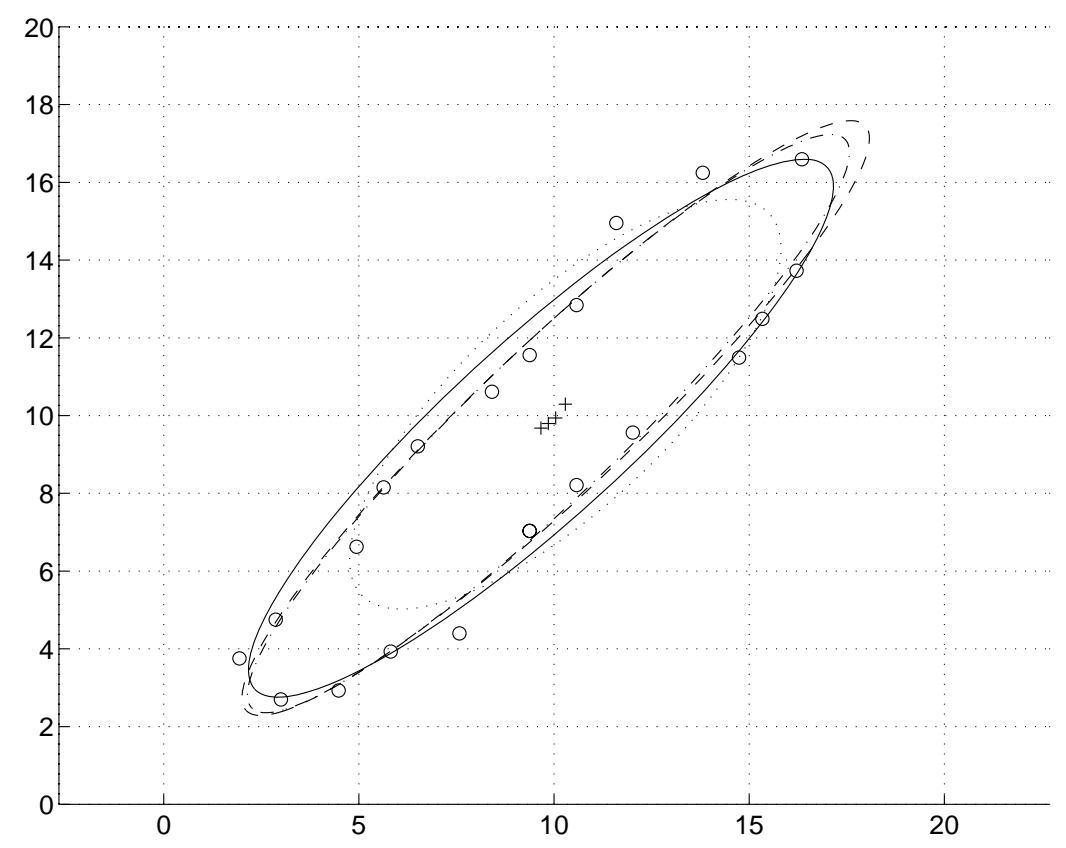

Figure 6: comparison of different fits

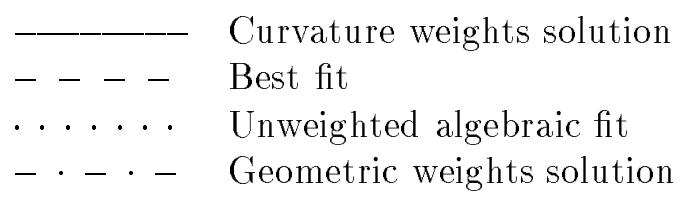

The sequence $\left(\mathbf{y}_{i}\right)$ may have a fixed point $\tilde{\mathbf{y}}=\mathbf{y}_{\infty}$ without solving (17), since the conditions for critical points $\tilde{\mathbf{x}}$ and $\tilde{\mathbf{y}}$ are different for the two equations. To show this, we shall use the notation $d \mathbf{z}$ for an infinitesimal change of $\mathbf{z}$. For all $d \mathbf{x}$ with $\tilde{\mathbf{x}}^{\mathrm{T}} d \mathbf{x}=0$ the following holds

$$
2\left(\tilde{\mathbf{x}}^{\mathrm{T}} G^{\mathrm{T}} d G \tilde{\mathbf{x}}+\tilde{\mathbf{x}}^{\mathrm{T}} G^{\mathrm{T}} G d \mathbf{x}\right)=d\|G \tilde{\mathbf{x}}\|^{2}=0
$$

for equation (17). Whereas for equation (18) and $\tilde{\mathbf{y}}^{\mathrm{T}} d \mathbf{y}=0$ the condition is

$$
2\left(\tilde{\mathbf{y}}^{\mathrm{T}} G^{\mathrm{T}} G d \mathbf{y}\right)=d\|G \tilde{\mathbf{y}}\|^{2}=0 .
$$

This problem is common to all iterative algebraic solutions of this kind, so no matter how good the weights approximate the real geometric distances, we may not generally expect that the sequence of estimated ellipses converges to the optimal solution.

We give a simple example for a fixed point of the iteration scheme (18), which does not solve (17). For $\mathbf{z}=(x, y)^{\mathrm{T}}$ consider

$$
G=\left(\begin{array}{cc}
2 & 0 \\
-4 y & 4
\end{array}\right)
$$

then $\mathbf{z}_{0}=(1,0)^{\mathrm{T}}$ is a fixed point of $(18)$, but $\mathbf{z}=(0.7278,0.6858)^{\mathrm{T}}$ is the solution of $(17)$.

Another severe problem with iterative algebraic methods is the lack of convergence in the general case-especially if the problem is ill-conditioned. We will shortly examine the 
solution of (18) for small changes to $G$. Let

$$
\begin{aligned}
& G=U \Sigma V^{\mathrm{T}} \\
& \bar{G}=G+d G=\bar{U} \bar{\Sigma} \bar{V}^{\mathrm{T}}
\end{aligned}
$$

and denote with $\sigma_{1}, \ldots, \sigma_{n}$ the singular values in descending order, with $\mathbf{v}_{i}$ the associated right singular vectors - where $\mathbf{v}_{n}$ is the solution of equation (18) for $G$. Then we may bound $\left\|d \mathbf{v}_{n}\right\|=\left\|\overline{\mathbf{v}}_{n}-\mathbf{v}_{n}\right\|$ as follows. First, we define

$$
\begin{aligned}
\lambda & =\bar{V}^{\mathrm{T}} \mathbf{v}_{n} \quad \text { thus }\|\lambda\|=1 \\
\mu & =1-\lambda_{n}^{2} \\
\varepsilon & =\|d G\|
\end{aligned}
$$

and note that

$$
\sigma_{i}-\varepsilon \leq \bar{\sigma}_{i} \leq \sigma_{i}+\varepsilon \text { for all } i
$$

We may conclude

$$
\|\bar{\Sigma} \lambda\|=\|\bar{U} \bar{\Sigma} \lambda\|=\left\|\bar{G} \mathbf{v}_{n}\right\|
$$

and

$$
\left\|\bar{G} \mathbf{v}_{n}\right\| \leq\left\|G \mathbf{v}_{n}\right\|+\left\|d G \mathbf{v}_{n}\right\| \leq \sigma_{n}+\varepsilon
$$

thus

$$
\sum_{i=1}^{n} \lambda_{i}^{2} \bar{\sigma}_{i}^{2} \leq \sigma_{n}^{2}+2 \varepsilon \sigma_{n}+\varepsilon^{2} .
$$

Using that $\bar{\sigma}_{i} \geq \bar{\sigma}_{n-1}$ for $i \leq n-1$, and that $\|\lambda\|=1$, we simplify the above expression to

$$
\left(1-\lambda_{n}^{2}\right) \bar{\sigma}_{n-1}^{2}+\lambda_{n}^{2} \bar{\sigma}_{n}^{2} \leq \sigma_{n}^{2}+2 \varepsilon \sigma_{n}+\varepsilon^{2} .
$$

Assuming that $\sigma_{n} \neq 0$ (otherwise the solution is exact) and $\varepsilon \leq \sigma_{n}$, we have

$$
\sigma_{i}^{2}-2 \varepsilon \sigma_{i}+\varepsilon^{2} \leq \bar{\sigma}_{i}^{2}
$$

Applying this to inequality (19), we get

$$
\mu \leq \frac{4 \varepsilon \sigma_{n}}{\bar{\sigma}_{n-1}^{2}-\bar{\sigma}_{n}^{2}} .
$$

Note that

$$
\left\|\mathbf{v}_{n}-\overline{\mathbf{v}}_{n}\right\|^{2}=\left\|\lambda-(0, \ldots, 1)^{\mathrm{T}}\right\|^{2}=\left(\lambda_{n}-1\right)^{2}+\left(1-\lambda_{n}^{2}\right)=2\left(1-\lambda_{n}\right) .
$$

Assuming that $\left\|\mathbf{v}_{n}-\overline{\mathbf{v}}_{n}\right\|$ is small (and thus $\lambda_{n} \approx 1, \mu \approx 0$ ), then we may write $\sigma$ for $\bar{\sigma}$ and $\mu=\left(1+\lambda_{n}\right)\left(1-\lambda_{n}\right) \approx 2\left(1-\lambda_{n}\right)$; thus we finally get

$$
\left\|\mathbf{v}_{n}-\overline{\mathbf{v}}_{n}\right\| \leq \sqrt{\frac{4 \varepsilon \sigma_{n}}{\sigma_{n-1}^{2}-\sigma_{n}^{2}}} \leq \sqrt{\frac{2 \varepsilon}{\sigma_{n-1}-\sigma_{n}}} .
$$

This shows that the convergence behavior of iterative algebraic methods depends on how well - in a figurative interpretation - the solution vector $\tilde{\mathbf{v}}_{n}$ is separated from its hyperplane with respect to the residual norm $\|G \mathbf{v}\|$. If $\tilde{\sigma}_{n-1} \approx \tilde{\sigma}_{n}$, the solution is poorly determined, and the algorithm may not converge. 


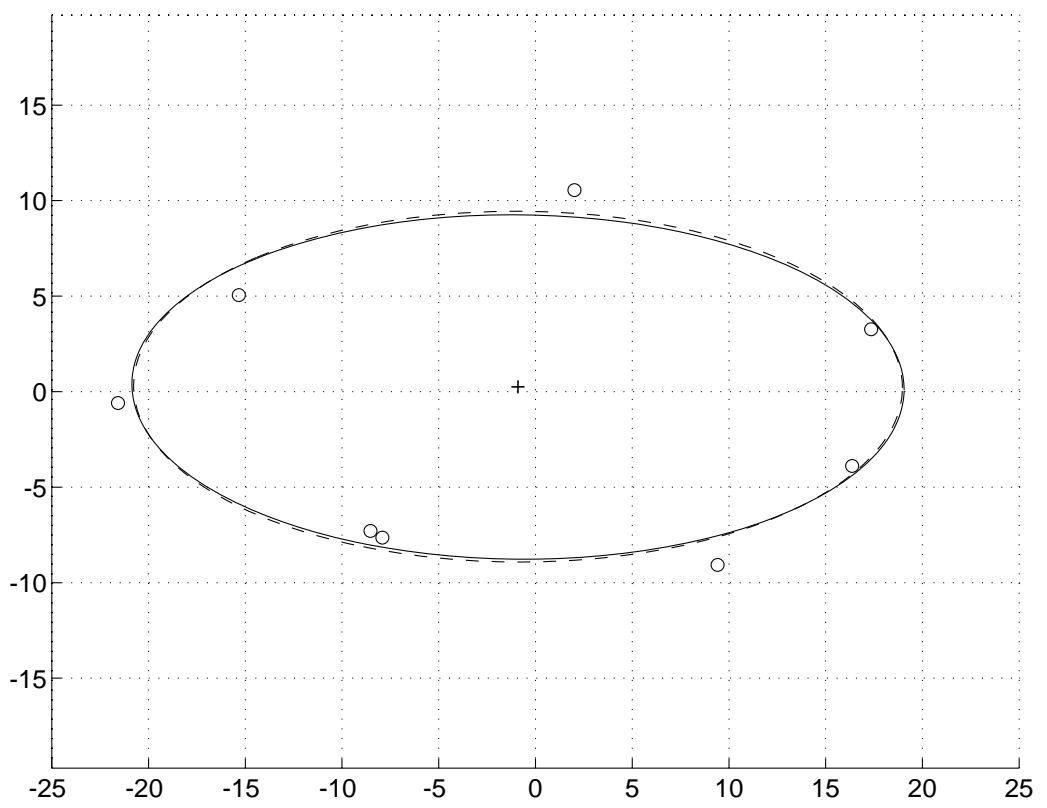

Figure 7: geometric weight fit vs. best fit

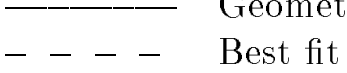

While the analytical results are not encouraging, we obtained solutions close to the optimum using the geometric-weight algorithm for several examples. Figure 7 shows the geometric-weight (solid) and the best (dashed) solution for such an example. Note that the calculation of geometric distances is relatively expensive, so a pragmatic way to limit the cost is to perform a fixed number of iterations, since convergence is not guaranteed anyway.

Appendix C.1 lists the points to be approximated, resulting in a residual norm of 2.784, compared to 2.766 for the best geometric fit. Since the algebraic method minimizes $w_{i} Q_{i}$ in the least squares sense, it remains to check that $w_{i} Q_{i}$ is proportional to the geometric distance $d_{i}$ for the estimated conic. We compute

$$
\begin{aligned}
M_{i} & =\left|\left(w_{i} Q_{i}\right) / d_{i}\right| \\
h_{i} & =1-M_{i} /\|M\|_{\infty}
\end{aligned}
$$

and find-as expected-that $\|\mathbf{h}\|=1.1 E-5$.

\subsection{Circle weight algorithm}

The main difficulty with above algebraic methods is that the solution may be any conicnot necessarily an ellipse. To cope with this case, we extend the system by weighted equations, which favour circles and non-eccentric ellipses:

$$
\begin{aligned}
\omega\left(a_{11}-a_{22}\right) & \approx 0 \\
\omega 2 a_{12} & \approx 0 .
\end{aligned}
$$


Note that these equations are Euclidean-invariant only if $\omega$ is the same in both equations, and the problem is solved in the least squares sense. What we are really minimizing in this case is

$$
\omega^{2}\left(\left(a_{11}-a_{22}\right)^{2}+4 a_{12}^{2}\right)=\omega^{2}\left(\lambda_{1}-\lambda_{2}\right)^{2}
$$

hence, the constraints (22) and (23) are equivalent to the equation

$$
\omega\left(\lambda_{1}-\lambda_{2}\right) \approx 0 .
$$

The weight $\omega$ is fixed by

$$
\omega=\epsilon f\left(\left(\lambda_{1}-\lambda_{2}\right)^{2}\right),
$$

where $\epsilon$ is a parameter representing the badness of eccentric ellipses, and

$$
f:[0, \infty[\rightarrow[0, \infty[\text { continuous, strictly increasing. }
$$

The larger $\epsilon$ is chosen, the larger will $\omega$ be, and thus the more important are equations $(22-23)$, which make the solution be more circle-shaped. The parameter $\omega$ is determined iteratively (starting with $\omega_{0}=0$ ), where following conditions hold

$$
0=\omega_{0} \leq \omega_{2} \leq \ldots \leq \omega \leq \ldots \leq \omega_{3} \leq \omega_{1}
$$

Thus, the larger the weight $\omega$, the less eccentric the ellipse; we prove this in the following. Given weights $\chi<\omega$ and

$$
F=\left(\begin{array}{cccccc}
1 & 0 & -1 & 0 & 0 & 0 \\
0 & 1 & 0 & 0 & 0 & 0
\end{array}\right),
$$

then we find solutions $\mathbf{x}$ and $\mathbf{z}$ for the equations

$$
\begin{aligned}
& \left\|\left(\begin{array}{c}
B \\
\chi F
\end{array}\right) \mathrm{x}\right\|=\min \\
& \left\|\left(\begin{array}{c}
B \\
\omega F
\end{array}\right) \mathrm{z}\right\|=\min
\end{aligned}
$$

respectively. It follows that

$$
\begin{aligned}
\|B \mathbf{x}\|^{2}+\chi^{2}\|F \mathbf{x}\|^{2} & \leq\|B \mathbf{z}\|^{2}+\chi^{2}\|F \mathbf{z}\|^{2} \\
\|B \mathbf{z}\|^{2}+\omega^{2}\|F \mathbf{z}\|^{2} & \leq\|B \mathbf{x}\|^{2}+\omega^{2}\|F \mathbf{x}\|^{2}
\end{aligned}
$$

and by adding

$$
\chi^{2}\|F \mathbf{x}\|^{2}+\omega^{2}\|F \mathbf{z}\|^{2} \leq \chi^{2}\|F \mathbf{z}\|^{2}+\omega^{2}\|F \mathbf{x}\|^{2}
$$

and since $\omega^{2}-\chi^{2}>0$

$$
\|F \mathbf{z}\|^{2} \leq\|F \mathbf{x}\|^{2},
$$

so that

$$
\bar{\omega}=\varepsilon f\left(\|F \mathbf{z}\|^{2}\right) \leq \varepsilon f\left(\|F \mathbf{x}\|^{2}\right)=\bar{\chi} .
$$

This completes the proof, since $f$ was chosen strictly increasing. One obvious choice for $f$ is the identity function, which was used in our test programs. 


\section{Comparison of geometric algorithms}

The discussion of algorithms minimizing the geometric distance is somewhat different from the algebraic distance problems. The problems in the latter are primarily stability and "good-looking" solutions; the former must be viewed by their efficiency, too. Generally, the simple algebraic solution is orders of magnitude cheaper than the geometric counterparts (for accurate results about a factor 10-100); thus iterative algebraic methods are a valuable alternative. But a comparison between algebraic and geometric algorithms would not be very enlightening-because there is no objective criterion to decide which estimate is better. However, we may compare different nonlinear least square algorithms to compute the geometric fit with respect to stability and efficiency.

\subsection{Algorithms}

Several known nonlinear least square algorithms have been implemented:

1. Gauss-Newton (gauss)

2. Newton (newton)

3. Gauss-Newton with Marquardt modification (marq)

4. Variable projection (varpro)

5. Orthogonal distance regression (odr)

The odr algorithm (see [13], [14]) solves the implicit minimization problem

$$
\begin{aligned}
f\left(\mathbf{x}_{i}+\delta_{i}, \beta\right) & =0 \\
\sum_{i}\left\|\delta_{i}\right\|^{2} & =\min
\end{aligned}
$$

where

$$
f(\mathrm{x}, \beta)=\beta_{3}\left(x_{1}-\beta_{1}\right)^{2}+2 \beta_{4}\left(x_{1}-\beta_{1}\right)\left(x_{2}-\beta_{2}\right)+\beta_{5}\left(x_{2}-\beta_{2}\right)^{2}-1 .
$$

Whereas the gauss, newton, marq and varpro algorithms solve the problem

$$
Q\left(\mathrm{x}, \varphi_{1}, \varphi_{2}, \ldots, \varphi_{m}, z_{1}, z_{2}, r\right)=\sum_{i=1}^{m}\left[\left(x_{i 1}-x\left(\varphi_{i}\right)\right)^{2}+\left(x_{i 2}-y\left(\varphi_{i}\right)\right)^{2}\right]=\min .
$$

For $a \approx b$, the Jacobian matrix is nearly singular, so the gauss and newton algorithms are modified to apply Marquardt steps in this case. Not surprising, this modification makes all algorithms behave similar with respect to stability if the initial parameters are accurate and the problem is well posed.

The MatlaB procedure pare implements algorithms $1-3$, the MatlaB procedure varpro the variable projection algorithm, and the MATLAB procedure odr the orthogonal distance regression. The algorithms are described in the appendix.

\subsection{Results}

To appreciate the results given in table 1, it must be said that the varpro algorithm is written for any separable functional and cannot take profit from the sparsity of the Jacobian. The algorithms were tested with example data - each consisting of 8 pointsfor following problems (the data sets are listed in appendix C.2) 
1. Special set of points (14)

2. Uniformly distributed data in a square

3. Points on a circle

4. Points on an ellipse with $a / b=2$

5. Points on hyperbola branch

\begin{tabular}{|l|r|r|r|r|r|}
\hline & gauss & newton & marq & varpro & \multicolumn{1}{c|}{ odr } \\
\hline Special & 146 & $\underline{85}$ & 468 & 1146 & $\diamond$ \\
Random & $\diamond$ & $\diamond$ & $\diamond$ & $\underline{2427}$ & $\diamond$ \\
Circle & 22 & 22 & 22 & 36 & $\underline{7}$ \\
Circle+ & 86 & $\underline{67}$ & 189 & 717 & 69 \\
Ellipse & $\underline{30}$ & 37 & 67 & 143 & 41 \\
Ellipse+ & 186 & $\diamond$ & 633 & 1977 & $\underline{103}$ \\
Hyperbola & 22 & 22 & 22 & 36 & $\underline{10}$ \\
Hyperbola+ & $\diamond$ & $\diamond$ & $\diamond$ & $\diamond$ & $\diamond$ \\
\hline
\end{tabular}

Table 1: Geometric fit with initial parameters of algebraic circle

\#flops/1000, minimum is underlined

' $\diamond$ if non-convergence

The tests with points on a conic were done both with and without perturbations. For table 1, the initial parameters were derived from the algebraically best fitting circle (radius $r_{a}$, center $z_{a}$ ); initial center $z_{0}=z_{a}$, axes $a_{0}=r_{a}, b_{0}=r_{a}$ and $\alpha_{0}=0$. Note that these initial values are somewhat rudimentary, so they serve to check the algorithms' stability, too. Table 1 shows the number of flops (in 1000) for the respective algorithm and problem; the smallest number is underlined. If the algorithm didn't terminate after 100 steps, it was assumed non-convergent and a ' $\diamond$ ' is shown instead. Table 2 contains the results if the initial parameters were obtained from the Bookstein algorithm.

Table 2 shows that all algorithms converge quickly with the more accurate initial data for exact conics. For the perturbed ellipse data, it's primarily the newton algorithm which profits from the starting values close to the solution. Note further that the newton algorithm does not find the correct solution for the special data, since the algebraic estimation - which serves as initial approximation - is completely different from the geometric solution.

General conclusions from this (admittedly) small test series are

- All algorithms are prohibitively expensive compared to the simple algebraic solution (factor 10-100).

- If the problem is well posed, and the accuracy of the result should be high, the newton method applied to the parameterized algorithm is the most efficient.

- The odr algorithm—although a simple general-purpose optimizing scheme-is competitive with algorithms specifically written for the ellipse fitting problem. If one 


\begin{tabular}{|l|r|r|r|r|r|}
\hline & gauss & newton & marq & varpro & \multicolumn{1}{c|}{ odr } \\
\hline Special & $\underline{165}$ & 896 & 566 & 1506 & $\diamond$ \\
Random & $\diamond$ & $\diamond$ & $\diamond$ & $\underline{2819}$ & $\diamond$ \\
Circle & 32 & 32 & 32 & 102 & $\underline{7}$ \\
Circle+ & 76 & $\underline{63}$ & 145 & 574 & 66 \\
Ellipse & 22 & 22 & 22 & 112 & $\underline{7}$ \\
Ellipse+ & 161 & $\underline{40}$ & 435 & 1870 & 74 \\
Hyperbola & $\diamond$ & $\diamond$ & $\diamond$ & $\underline{1747}$ & $\diamond$ \\
Hyperbola+ & $\diamond$ & $\diamond$ & $\diamond$ & $\underline{2986}$ & $\diamond$ \\
\hline
\end{tabular}

Table 2: Geometric estimation with initial data of algebraic ellipse

\#flops/1000, minimum is underlined

' $\diamond$ if non-convergence

takes into consideration further, that we didn't use a highly optimized odr procedure, the method of solution is surprisingly simple and efficient.

- The varpro algorithm seems to be the most expensive. Reasons for its inefficiency are that most parameters are non-linear and that the algorithm does not make use of the special matrix structure for this problem.

\section{References}

[1] Vaughan Pratt, Direct Least Squares Fitting of Algebraic Surfaces, ACM J. Computer Graphics, Volume 21, Number 4, July 1987

[2] M. G. Cox, A. B. Forbes, Strategies for Testing Assessment Software, NPL Report DITC 211/92

[3] G. Golub, Ch. Van Loan, Matrix Computations (second ed.), The Johns Hopkins University Press, Baltimore, 1989

[4] D. Sourlier, A. Bucher, Normgerechter Best-fit-Algorithmus für FreiformFlächen oder andere nicht-reguläre Ausgleichs-Flächen in Parameter-Form, Technisches Messen 59, (1992), pp. 293-302

[5] H. Sрӓтн, Orthogonal Least Squares Fitting with Linear Manifolds, Numer. Math., $48: 441-445,1986$.

[6] Lyle B. Smith, The use of man-machine interaction in data fitting problems, TR No. CS 131, Stanford University, March 1969 (thesis)

[7] Y. Vladimirovich Linnik, Method of least squares and principles of the theory of observations, New York, Pergamon Press, 1961

[8] Charles L. Lawson, Contributions to the Theory of Linear Least Maximum Approximation, Dissertation University of California, Los Angeles, 1961 
[9] Fred L. Bookstein, Fitting Conic Sections to Scattered Data, Computer Graphics and Image Processing 9, (1979), pp. 56-71

[10] G. H. Golub, V. Pereyra, The Differentiation of Pseudo-Inverses and Nonlinear Least Squares Problems whose Variables Separate, SIAM J. Numer. Anal. 10, No. 2, (1973); pp. 413-432

[11] M. Heidari, P. C. Heigold, Determination of Hydraulic Conductivity Tensor Using a Nonlinear Least Squares Estimator, Water Resources Bulletin, June 1993, pp. 415424

[12] W. Gander, U. von Matt, Some Least Squares Problems, Solving Problems in Scientific Computing Using Maple and Matlab, W. Gander and J. Hřebíček ed., 251266, Springer-Verlag, 1993.

[13] Paul T. Boggs, Richard H. Byrd and Robert B. Schnabel, A stable and efficient algorithm for nonlinear orthogonal distance regression, SIAM Journal Sci. and Stat. Computing 8(6):1052-1078, November 1987.

[14] Paul T. Boggs, Richard H. Byrd, Janet E. Rogers and Robert B. SchnABEL, User's Reference Guide for ODRPACK Version 2.01-Software for Weighted Orthogonal Distance Regression, National Institute of Standards and Technology, Gaithersburg, June 1992.

[15] P. E. Gill, W. Murray and M. H. Wright, Practical Optimization, Academic Press, New York, 1981.

[16] Gene H. Golub, Alan Hoffmann, G. W. Stewart, A Generalization of the Eckart-Young-Mirsky Matrix Approximation Theorem, Linear Algebra and its Appl. $88 / 89: 317-327(1987)$ 


\section{A Algorithms}

\section{A.1 Gauss-Newton with Marquardt correction}

Given parameters $\mathrm{x}=\left(\varphi_{1}, \ldots, \varphi_{m}, \alpha, a, b, z_{1}, z_{2}\right)^{\mathrm{T}}$, we consider the simplified equation of the Gauss-Newton method

$$
J \mathbf{h}=\left(\begin{array}{rr}
-a S & A \\
b C & B
\end{array}\right) \mathbf{h} \approx \mathbf{x} .
$$

Then, the equation for the same problem with Marquardt correction is

$$
\left(\begin{array}{l}
J \\
\Lambda
\end{array}\right) \mathbf{h} \approx\left(\begin{array}{l}
\mathrm{x} \\
\mathbf{0}
\end{array}\right) .
$$

If $\Lambda$ is chosen diagonal (e.g. $\Lambda=\lambda I$ ), we can triangularize the first $m$ colums of the extended system with $2 m$ Givens rotations. Thus, as in the case of the Gauss-Newton algorithm, the full QR algorithm has to be applied only to the last 5 columns.

The $\lambda_{i}$ parameter for the $i^{\text {th }}$ step is determined as follows: $\lambda_{i}=\nu^{k} \lambda_{i-1}(\nu>1, k \geq-1)$ where $k$ is chosen minimal so that $\left\|\mathbf{r}_{i}\right\| \leq\left\|\mathbf{r}_{i-1}\right\|$ holds for the residual $\mathbf{r}$.

\section{A.2 The Newton algorithm}

Using the terminology of the previous section, the equations for one Newton step may be written as

$$
\left(J^{\mathrm{T}} J+H\right) \mathbf{h}=-J^{\mathrm{T}} \mathbf{x},
$$

With parameter vector $\mathrm{x}=\left(\varphi_{1}, \ldots, \varphi_{m}, \alpha, a, b, z_{1}, z_{2}\right)^{\mathrm{T}}$, we may define $H$ by

$$
H_{j k}=\sum_{i} \mathbf{g}_{i}{ }^{\mathrm{T}} \frac{\partial^{2} \mathbf{g}_{i}}{\partial x_{j} \partial x_{k}} .
$$

For completeness, we'll list the second derivatives of the residual functions $\mathbf{g}_{i}$ :

$$
\begin{aligned}
\frac{\partial^{2} \mathbf{g}_{i}}{\partial \varphi_{j} \partial \varphi_{k}} & =\delta_{i j} \delta_{i k} Q(\alpha)\left(\begin{array}{c}
a \cos \varphi_{i} \\
b \sin \varphi_{i}
\end{array}\right) \\
\frac{\partial^{2} \mathbf{g}_{i}}{\partial \varphi_{j} \partial \alpha} & =-\delta_{i j} \dot{Q}(\alpha)\left(\begin{array}{c}
-a \sin \varphi_{i} \\
b \cos \varphi_{i}
\end{array}\right) \\
\frac{\partial^{2} \mathbf{g}_{i}}{\partial \varphi_{j} \partial a} & =-\delta_{i j} Q(\alpha)\left(\begin{array}{c}
\sin \varphi_{i} \\
0
\end{array}\right) \\
\frac{\partial^{2} \mathbf{g}_{i}}{\partial \varphi_{j} \partial b} & =-\delta_{i j} Q(\alpha)\left(\begin{array}{c}
0 \\
\cos \varphi_{i}
\end{array}\right) \\
\frac{\partial^{2} \mathbf{g}_{i}}{\partial \varphi_{j} \partial z_{k}} & =\mathbf{0} \\
\frac{\partial^{2} \mathbf{g}_{i}}{\partial \alpha^{2}} & =Q(\alpha)\left(\begin{array}{c}
a \cos \varphi_{i} \\
b \sin \varphi_{i}
\end{array}\right) \\
\frac{\partial^{2} \mathbf{g}_{i}}{\partial \alpha \partial a} & =-\dot{Q}(\alpha)\left(\begin{array}{c}
\cos \varphi_{i} \\
0
\end{array}\right)
\end{aligned}
$$




$$
\begin{aligned}
\frac{\partial^{2} \mathbf{g}_{i}}{\partial \alpha \partial b} & =-\dot{Q}(\alpha)\left(\begin{array}{c}
0 \\
\sin \varphi_{i}
\end{array}\right) \\
\frac{\partial^{2} \mathbf{g}_{i}}{\partial \alpha \partial z_{k}} & =\mathbf{0} \\
\frac{\partial^{2} \mathbf{g}_{i}}{\partial a^{2}} & =\frac{\partial^{2} \mathbf{g}_{i}}{\partial a \partial b}=\frac{\partial^{2} \mathbf{g}_{i}}{\partial a \partial z_{k}}=\frac{\partial^{2} \mathbf{g}_{i}}{\partial b^{2}}=\frac{\partial^{2} \mathbf{g}_{i}}{\partial b \partial z_{k}}=\frac{\partial^{2} \mathbf{g}_{i}}{\partial z_{j} \partial z_{k}}=\mathbf{0}
\end{aligned}
$$

The main disadvantage of the Newton method is that we have to solve a linear system with a non-sparse matrix, which is awfully conditioned for ill-posed problems.

\section{A.3 varpro-The variable projection algorithm}

This chapter only gives a survey on the algorithm; for a detailed description see [10]. The following problem is considered: Find optimal parameters $\hat{\mathbf{a}}=\left(\hat{a}_{1}, \ldots, \hat{a}_{n}\right)^{\mathrm{T}}, \hat{\alpha}=$ $\left(\hat{\alpha}_{1}, \ldots, \hat{\alpha}_{k}\right)^{\mathrm{T}}$ that minimize the nonlinear functional

$$
r(\mathbf{a}, \alpha)=\sum_{i=1}^{m}\left[y_{i}-\sum_{j=1}^{n} a_{j} \phi_{j}\left(\alpha ; t_{i}\right)\right]^{2} .
$$

Let

$$
\{\Phi\}_{i, j}=\phi_{j}\left(\alpha ; t_{i}\right), \quad i=1, \ldots, m ; j=1, \ldots, n .
$$

Then (29) can be written as

$$
r(\mathbf{a}, \alpha)=\|\mathbf{y}-\mathbf{\Phi}(\alpha) \mathbf{a}\|^{2}
$$

The idea is to minimize first a modified functional which depends only on the nonlinear parameters $\alpha$, and then proceed to obtain the linear parameters $\mathbf{a}$. In order to obtain the separation of variables, the modified functional

$$
r_{2}(\alpha)=\left\|\mathbf{y}-\Phi(\alpha) \Phi^{+}(\alpha) \mathbf{y}\right\|^{2}
$$

is considered, which is called the variable projection functional. Once optimal parameters $\hat{\alpha}$ have been obtained by minimizing (31), then the parameters â are obtained as a solution of $\boldsymbol{\Phi}(\hat{\alpha}) \mathbf{a} \approx \mathbf{y}$. This approach to finding a critical point requires an important hypothesis: not only must the involved functions be continuously differentiable, but the matrix $\boldsymbol{\Phi}(\alpha)$ must have constant rank for an open set containing the desired solution. If this condition does not hold, the pseudo-inverse of $\boldsymbol{\Phi}$ is a discontinuous function in the critical point.

For any given $\alpha$ we have the minimal least squares solution

$$
\hat{\mathbf{a}}(\alpha)=\Phi^{+}(\alpha) \mathbf{y} .
$$

Thus,

$$
\min _{\mathbf{a}} r(\mathbf{a}, \alpha)=r(\hat{\mathbf{a}}, \alpha)=\left\|\mathbf{y}-\boldsymbol{\Phi}(\alpha) \Phi^{+}(\alpha) \mathbf{y}\right\|^{2}=r_{2}(\alpha) .
$$

The modified functional is then the variable projection functional that we mentioned earlier. See [10] for a proof that-under the assumption mentioned above- $(\hat{\mathbf{a}}, \hat{\alpha})$ is a minimizer for $r$ if and only if $\hat{\alpha}$ is a minimizer for $r_{2}$. To apply the Gauss-Newton algorithm (possibly with Marquardt correction), it is necessary to differentiate the pseudo-inverse; this is the primary contribution of [10]. Further, the developed formulas allow to study the stability of the solution of perturbed linear least square problems. 


\section{A.4 odr - The Orthogonal Distance Regression algorithm}

The odr algorithm provides an elegant method to the general solution of the problem

$$
\begin{aligned}
y_{i}+\epsilon_{i} & =f\left(\mathbf{x}_{i}+\delta_{i}, \beta\right) \\
\sum_{i}\left\|\delta_{i}\right\|^{2}+\epsilon_{i}^{2} & =\min ,
\end{aligned}
$$

which may further be extended by a general weighting scheme. See [13] for details, and [14] for an implementation in ForTRAN. The form finally chosen results in the general ODR problem

$$
\min _{\beta, \delta} \sum_{i} w_{i}^{2}\left[\left(f\left(\mathbf{x}_{i}+\delta_{i}, \beta\right)-y_{i}\right)^{2}+\delta_{i}^{\mathrm{T}} D_{i}^{2} \delta_{i}\right]
$$

where $w_{i}>0$, and $D_{i}=\operatorname{diag}\left(d_{i j}\right)$ where $d_{i j}>0$.

The odr algorithm solves the problem (34) using Levenberg-Marquardt. The number of unknowns involved is the number of model parameters plus the number of data points. By exploiting sparsity, however, the algorithm has a per step computational effort similar to the Levenberg-Marquardt method for ordinary least squares.

The implicit problem

$$
\begin{aligned}
0 & =f\left(\mathbf{x}_{i}+\delta_{i}, \beta\right) \\
\sum_{i}\left\|\delta_{i}\right\|^{2} & =\min ,
\end{aligned}
$$

may be solved by a penalty function method (see e.g. [15]). The penalty function is

$$
P\left(\beta, \delta ; r_{k}\right)=\sum_{i} r_{k} f\left(\mathbf{x}_{i}+\delta_{i}, \beta\right)^{2}+\delta_{i}^{\mathrm{T}} D_{i}^{2} \delta_{i}
$$

with penalty parameter $r_{k}$. A sequence of unconstrained minimization problems

$$
\min _{\beta, \delta} P\left(\beta, \delta ; r_{k}\right)
$$

for a sequence of values $\left\{r_{k}\right\}$ results in weighted orthogonal distance regression problems (which are explicit)

$$
\epsilon_{i}=f\left(\mathbf{x}_{i}+\delta_{i}, \beta\right)
$$

and their solutions approach for $r_{k} \rightarrow \infty$ the solution of

$$
0=f\left(\mathbf{x}_{i}+\delta_{i}, \beta\right) .
$$

To apply this algorithm to the ellipse fitting problem, we may use the algebraic equation

$$
f(\beta)=\beta_{3}\left(x-\beta_{1}\right)^{2}+2 \beta_{4}\left(x-\beta_{1}\right)\left(y-\beta_{2}\right)+\beta_{5}\left(y-\beta_{2}\right)^{2}-1,
$$

which is simple to differentiate and does not require trigonometric functions.

\section{B Matla B Implementation}

Various small Matla B functions have been written for this paper, documented herein to provide an easy way to reproduce the results. 


\section{B.1 Utilities}

Linear least squares problem with special constraint

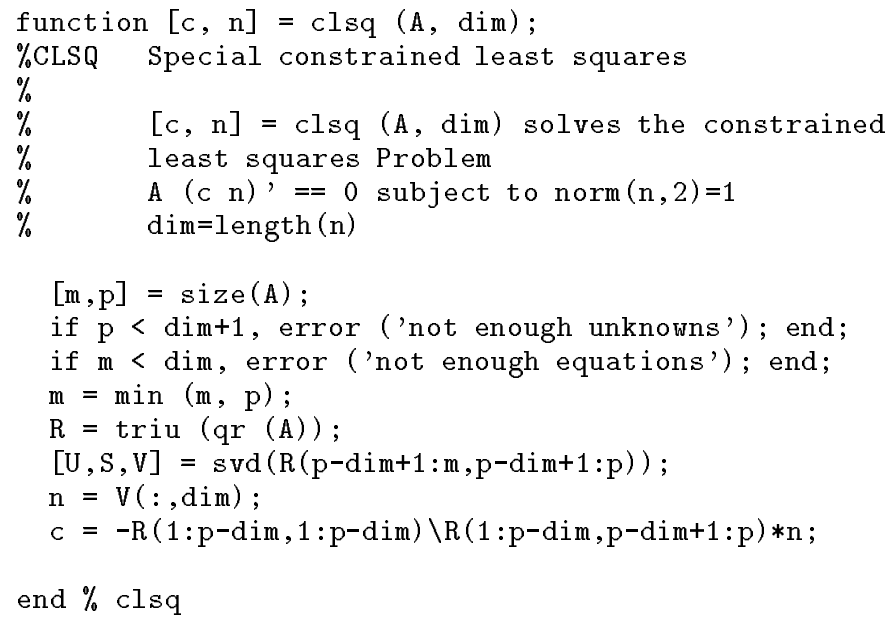

end \% drawcircle

Draw ellipse

function drawellipse ( $\mathrm{z}, \mathrm{a}, \mathrm{b}$, alpha, pat, OPTIONS) \%DRAWELLIPSE Draw ellipse

$\%$

$\%$ drawellipse (z, a, b, alpha, pat $\left\{{ }^{\prime}-'\right\}$, OPTIONS $\{[]\}$ )

$\%$ draws ellipse into current figure.

$\%$

$\% \mathrm{z}, \mathrm{a}, \mathrm{b}$, alpha: parameters of ellipse

$\%$ pat: pattern to be used

if (nargin < 6), OPTIONS = [2]; end;

if $($ nargin $<5)$, pat $=$ '-'; end; 


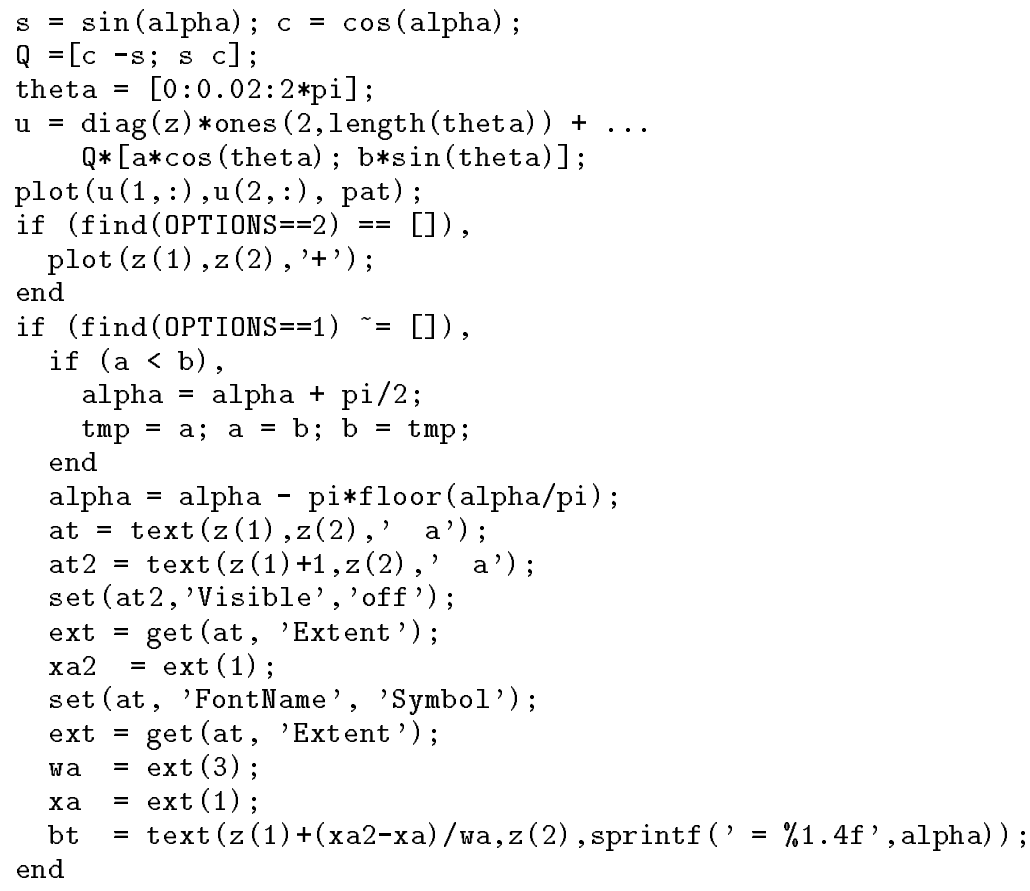

end \% drawellipse

Compute angles for Givens-Rotation matrix

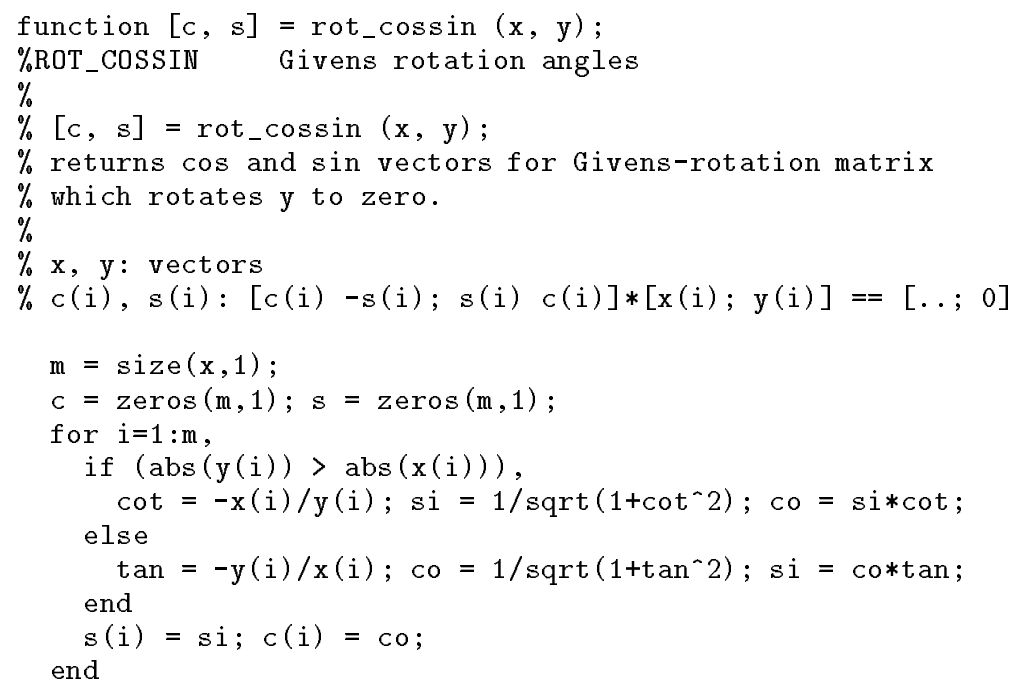

end $\%$ rot_cossin

Compute nearest points on ellipse to given points

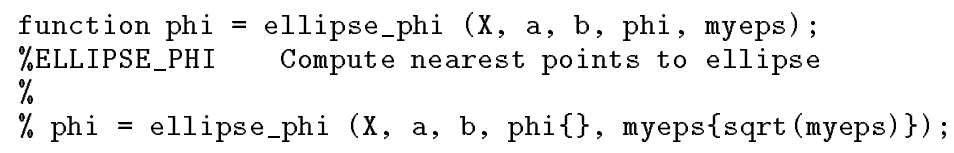




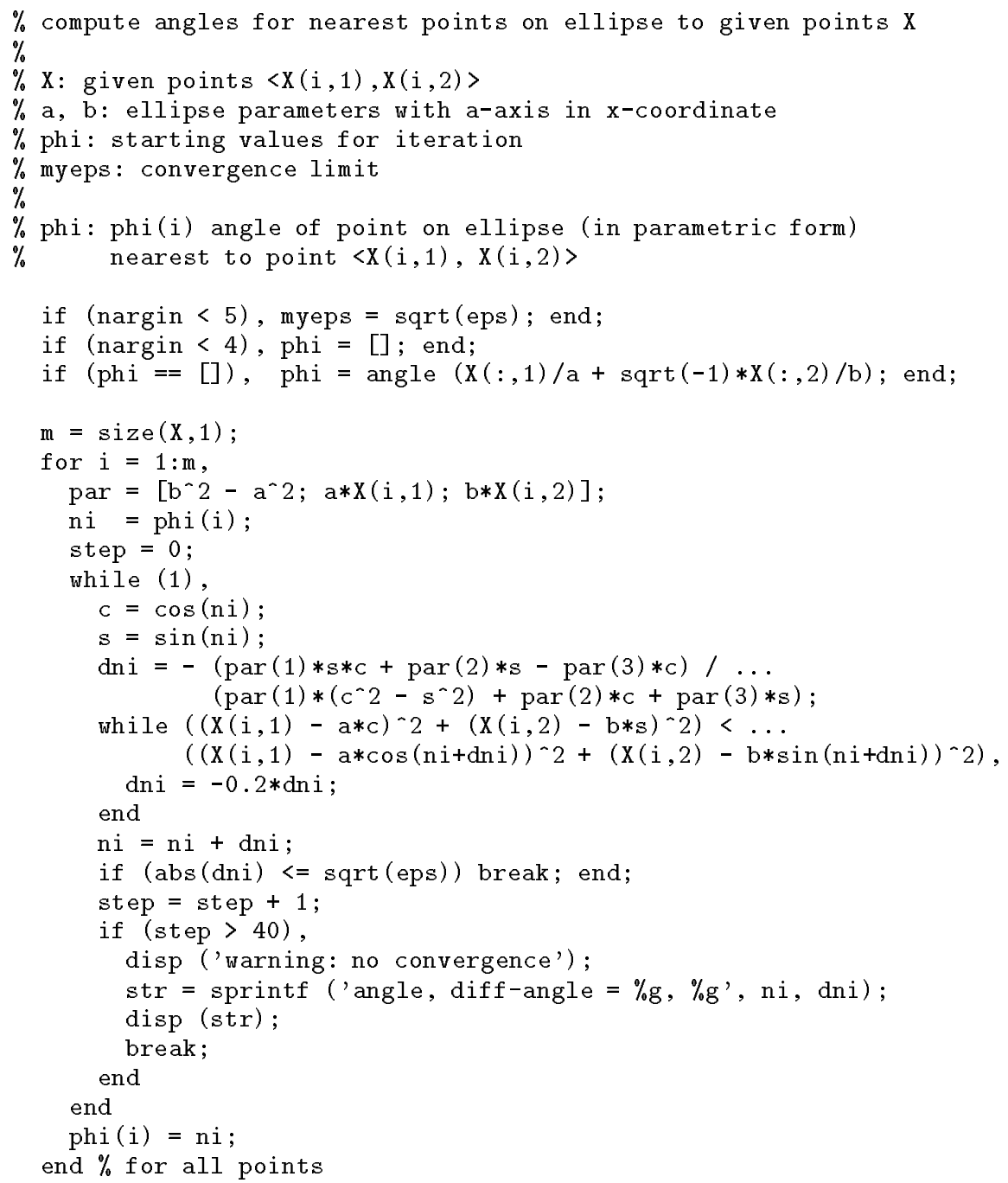

Compute distance vectors from given points to ellipse

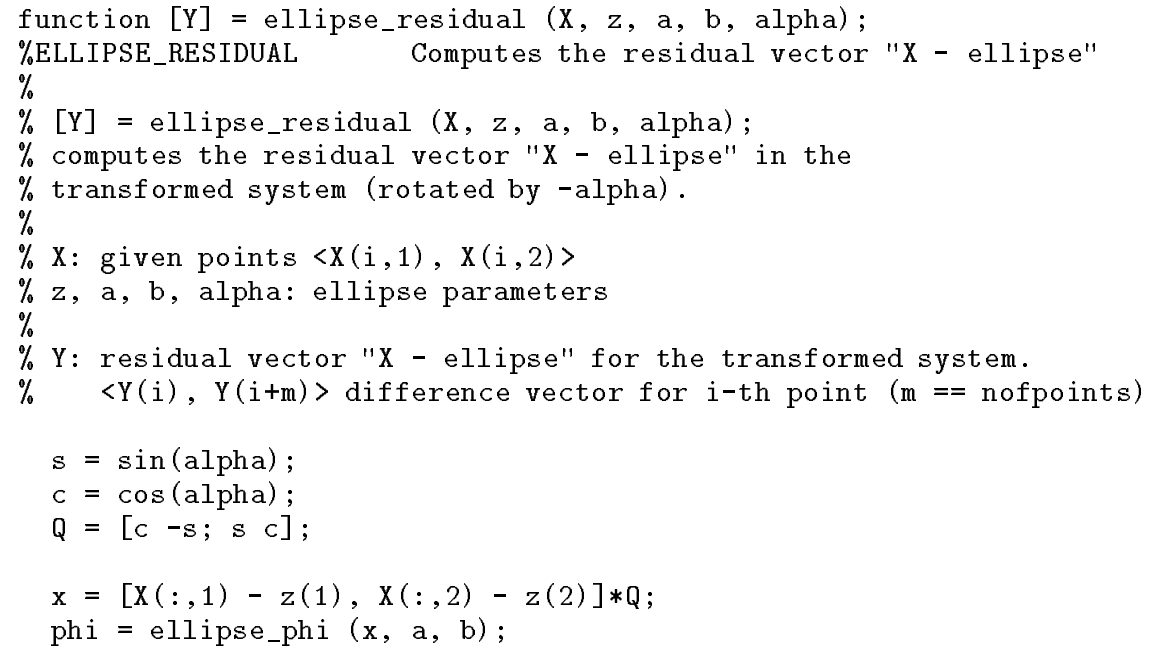


$\mathrm{Y}=\mathrm{x}-[\mathrm{a} * \cos (\mathrm{ph} i), \mathrm{b} * \sin (\mathrm{ph} i)] ;$

$\mathrm{Y}=[\mathrm{Y}(:, 1) ; \mathrm{Y}(:, 2)]$;

end \% ellipse_residual

Find ellipse parameters from algebraic equation

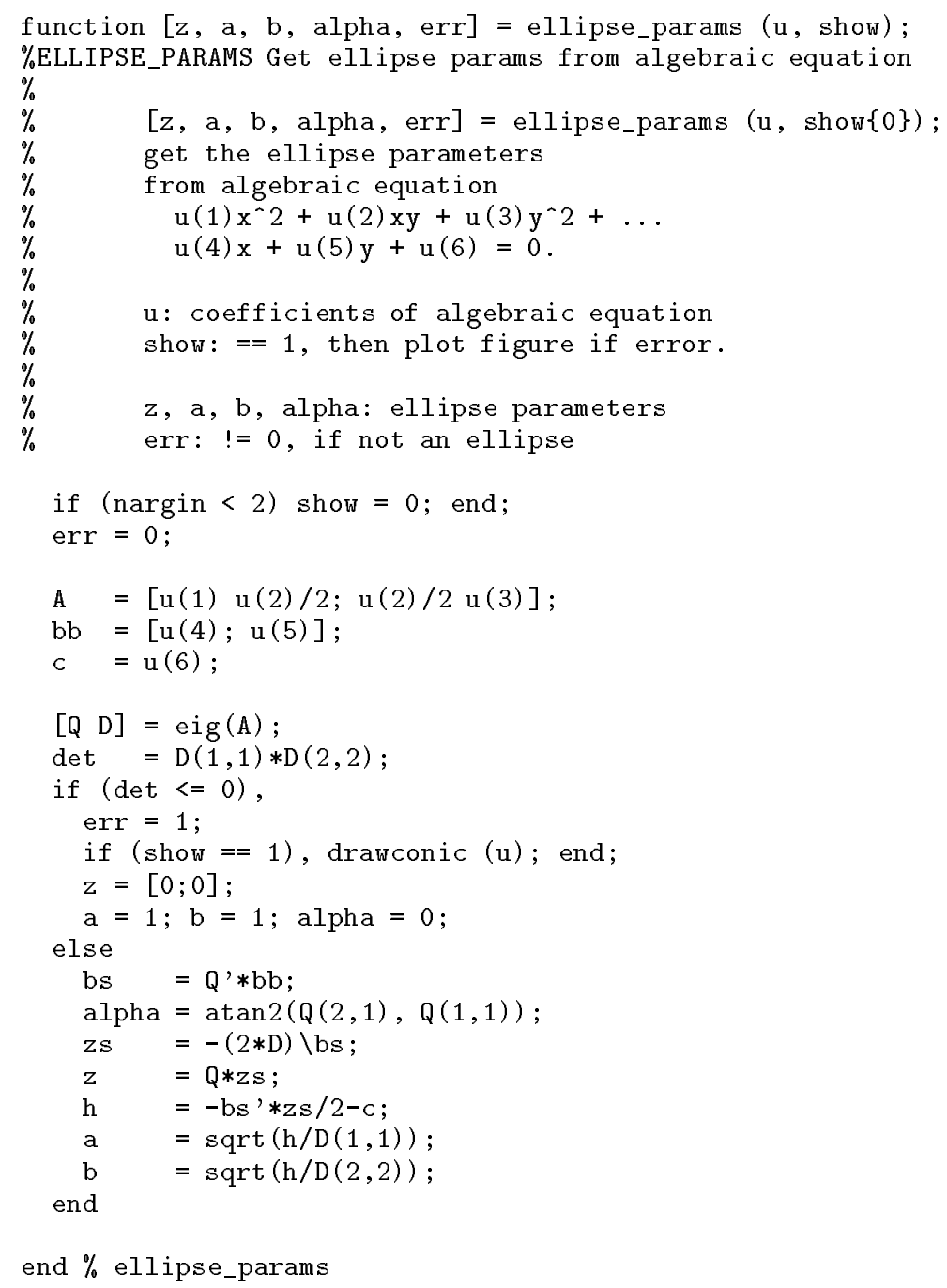

end \% ellipse_params

\section{B.2 Circle estimation}

Algebraic circle solution

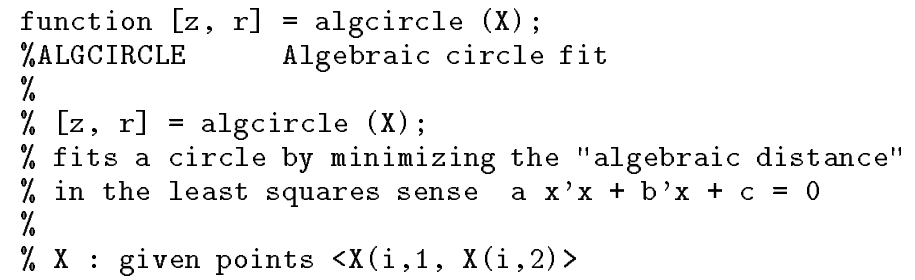


$\mathrm{B}=\left[\mathrm{X}(:, 1) \cdot{ }^{-} 2+\mathrm{X}(:, 2) \cdot^{-2} \mathrm{X}(:, 1) \quad \mathrm{X}(:, 2)\right.$ ones $\left.(\operatorname{size}(\mathrm{X}(:, 1)))\right]$;

$\left[\begin{array}{lll}\mathrm{U} & \mathrm{S} & \mathrm{V}\end{array}\right]=\operatorname{svd}(\mathrm{B})$;

$\mathrm{u}=\mathrm{V}(:, 4) ; \mathrm{a}=\mathrm{u}(1) ; \mathrm{b}=[\mathrm{u}(2) ; \mathrm{u}(3)] ; \mathrm{c}=\mathrm{u}(4)$;

$z=-b / 2 / a ; r=\operatorname{sqrt}\left(\operatorname{norm}(z)^{\wedge} 2-c / a\right) ;$

end $\%$ algcircle

Geometric circle solution in explicit form

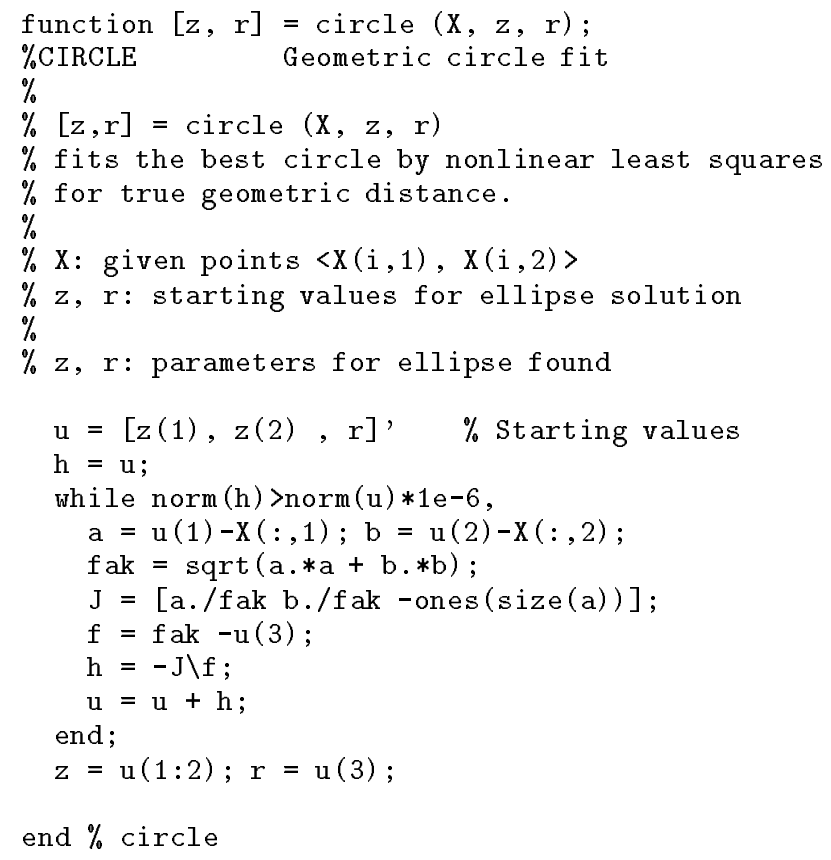

Geometric circle solution in parametric form

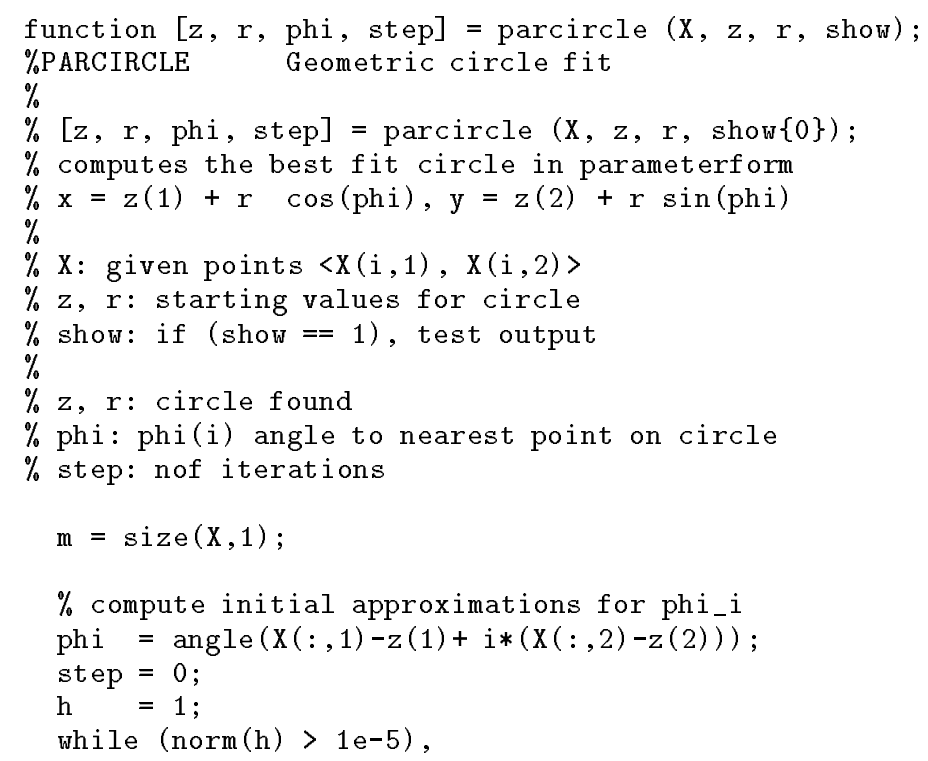




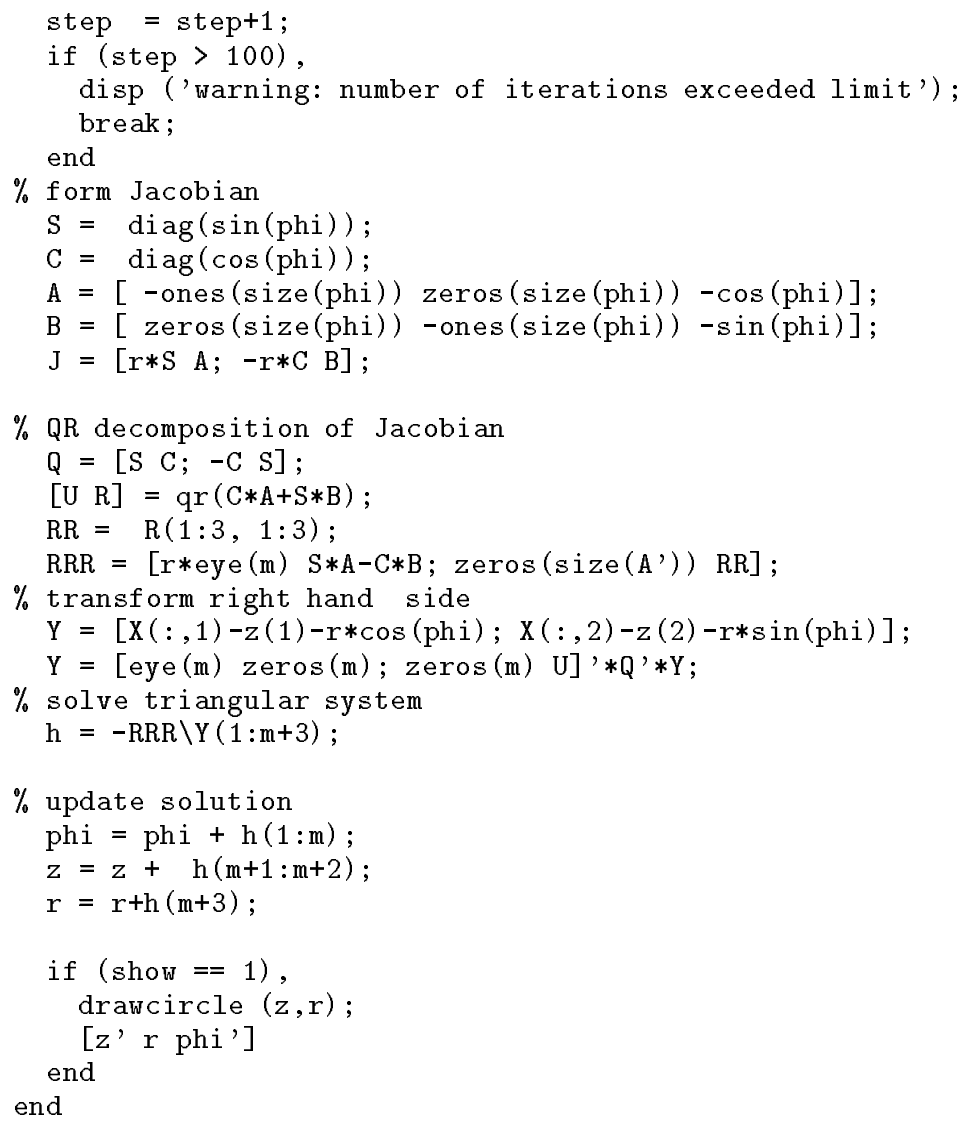

end $\%$ parcircle

\section{B.3 Algebraic ellipse estimation}

SVD algebraic ellipse estimation

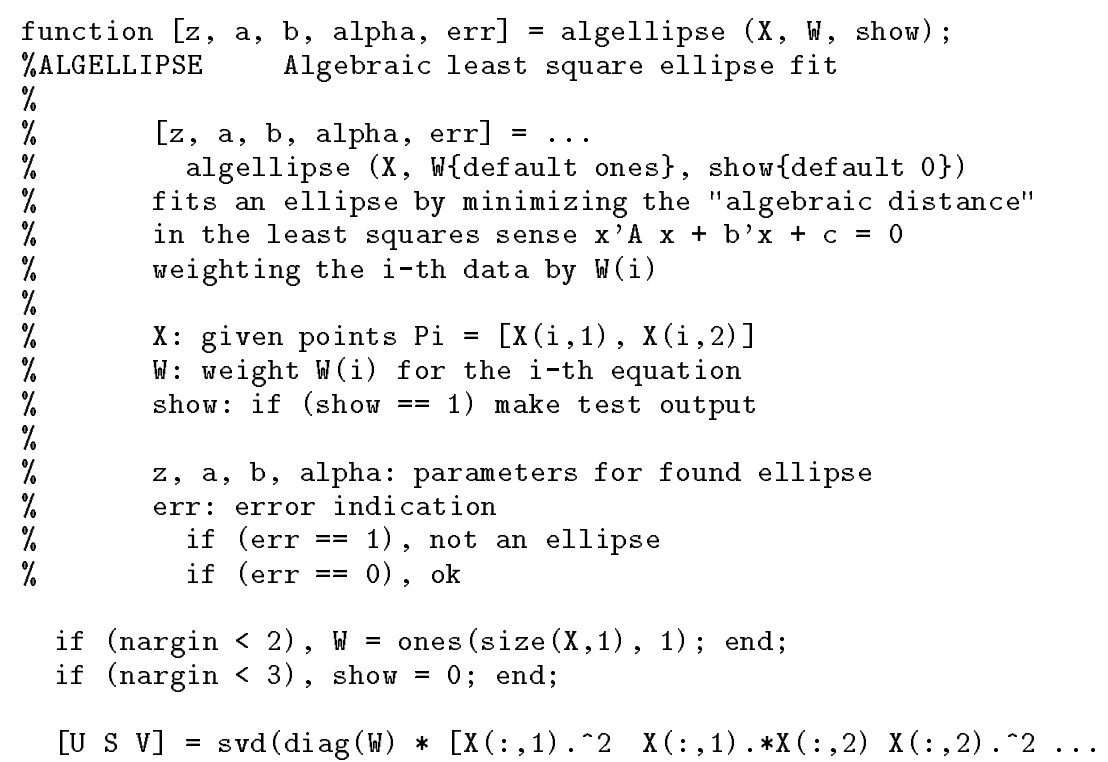


$\mathrm{u} \quad \mathrm{v}(:, 6)$;

$X(:, 1) X(:, 2)$ ones $(\operatorname{size}(X(:, 1)))])$;

$[z, a, b, a l p h a$, err $]=$ ellipse_params (u, show);

end \% algellipse

Linear algebraic ellipse estimation

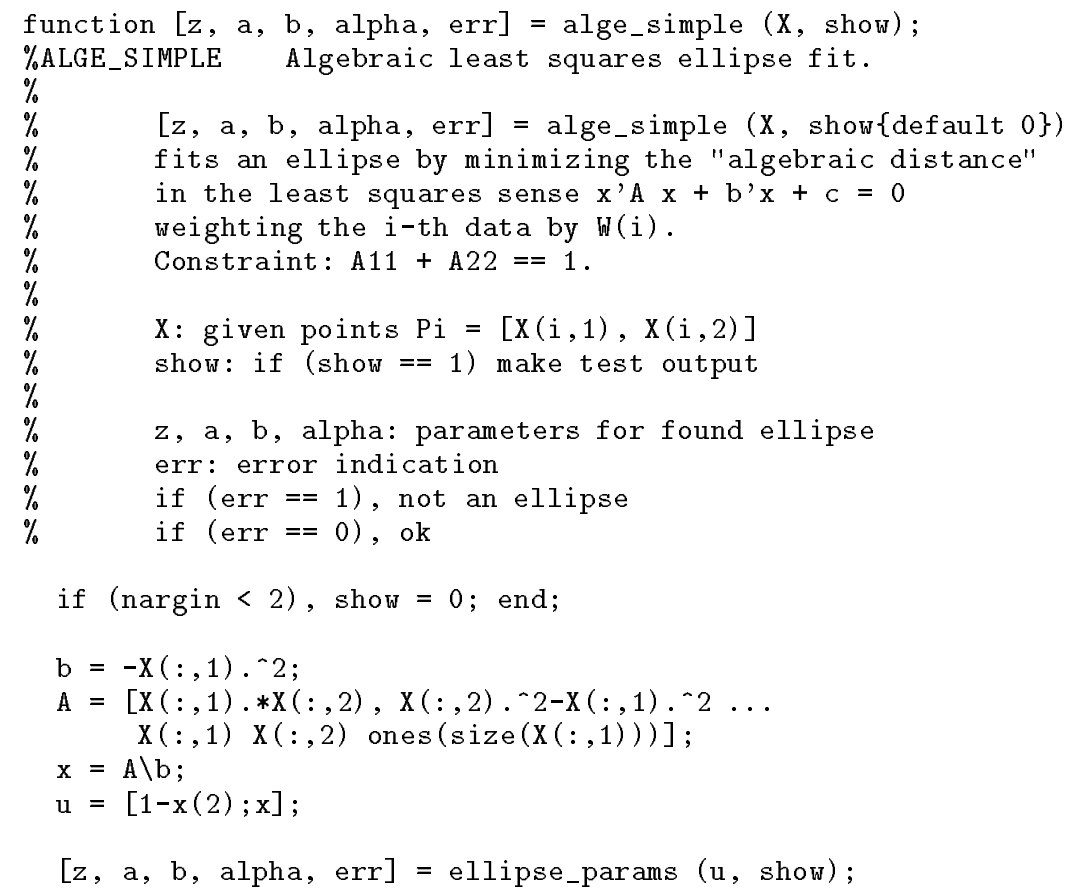

Bookstein algorithm

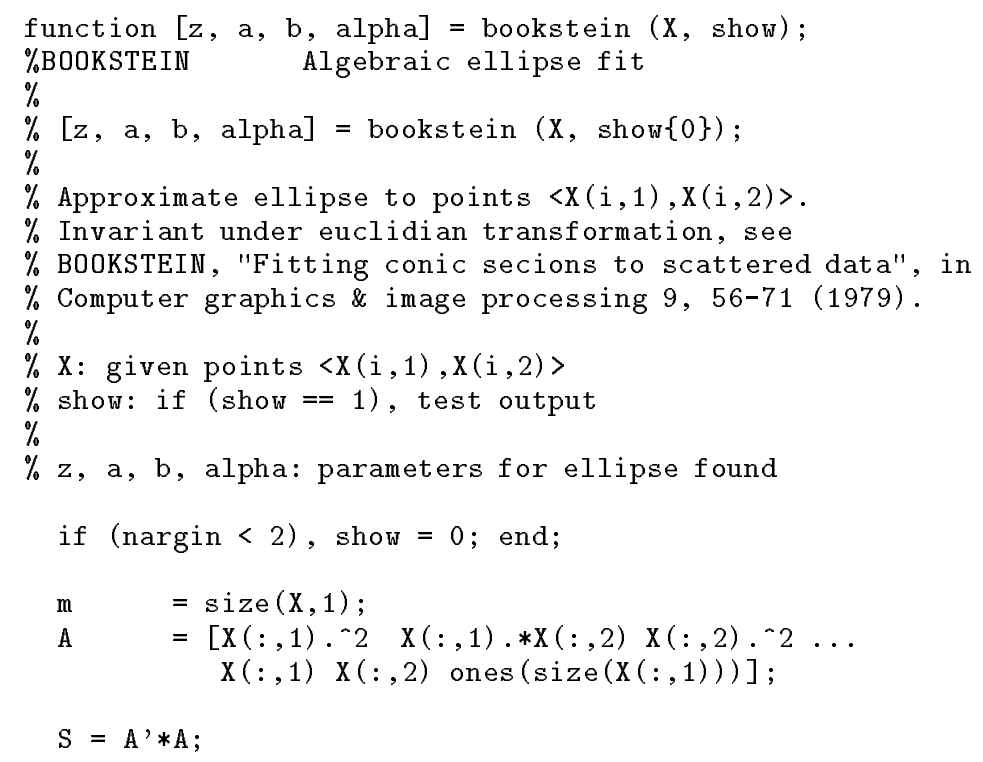




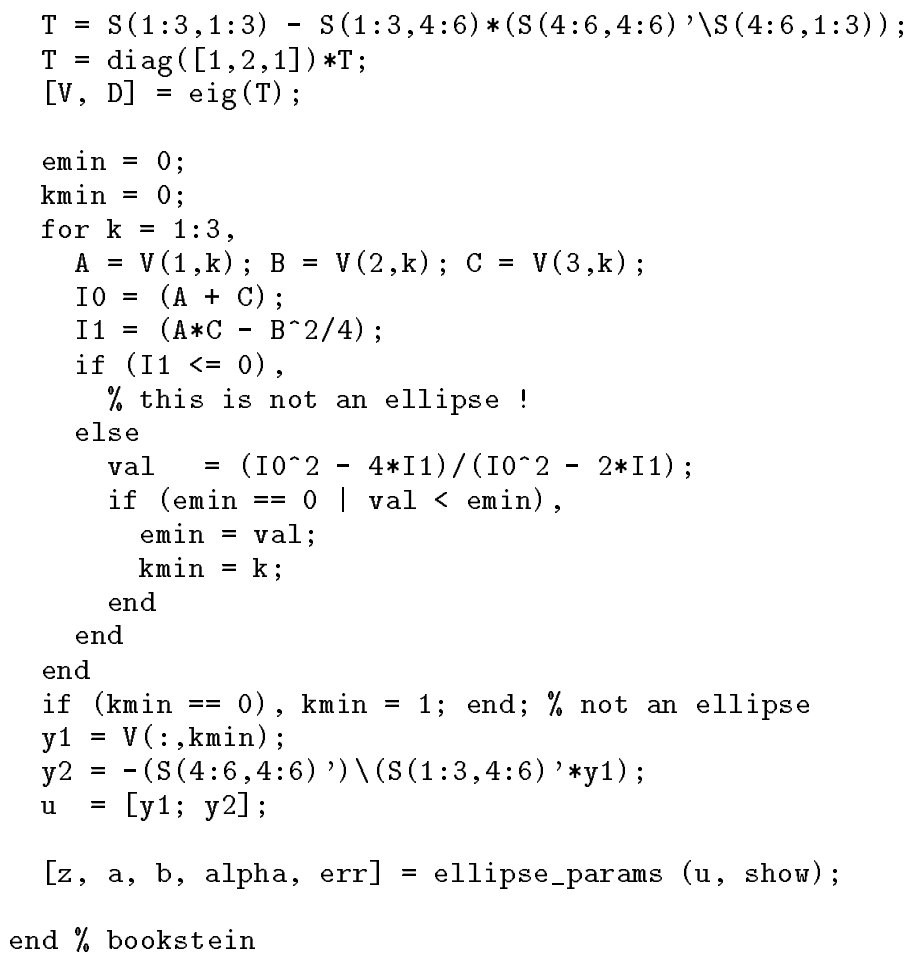

SVD solution for Bookstein constraint

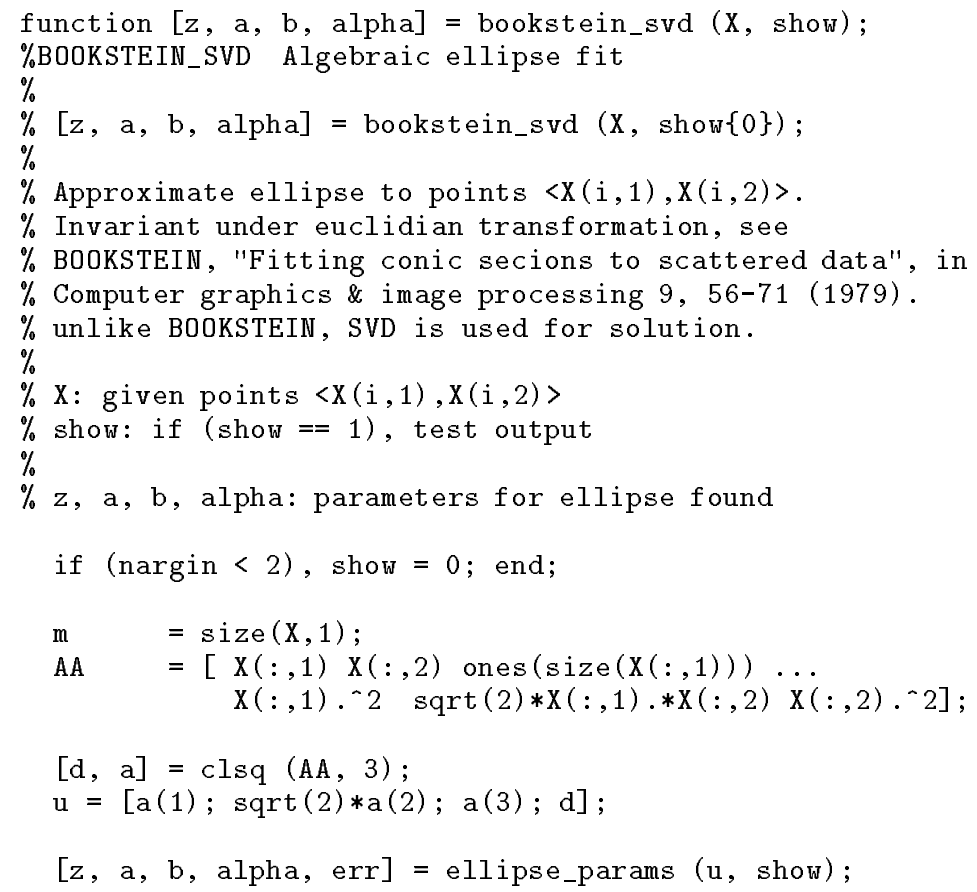

Curvature weights 
function $[z, a, b$, alpha, step $]=$ lyle (X, show);

$\%$ LYLE Iterative algebraic ellipse fit

$\%$

$\%[z, a, b$, alpha, step $]=1 y l e(X, \operatorname{show}\{0\}) ;$

$\%$ fit ellipse with algebraic method using curvature weights.

$\%$

$\% X$ : points given $\langle X(i, 1), X(i, 2)\rangle$

$\%$ show: if (show $==1$ ), test output

$\%$

$\%$ z, a, b, alpha: ellipse found

$\%$ step: nof iterations

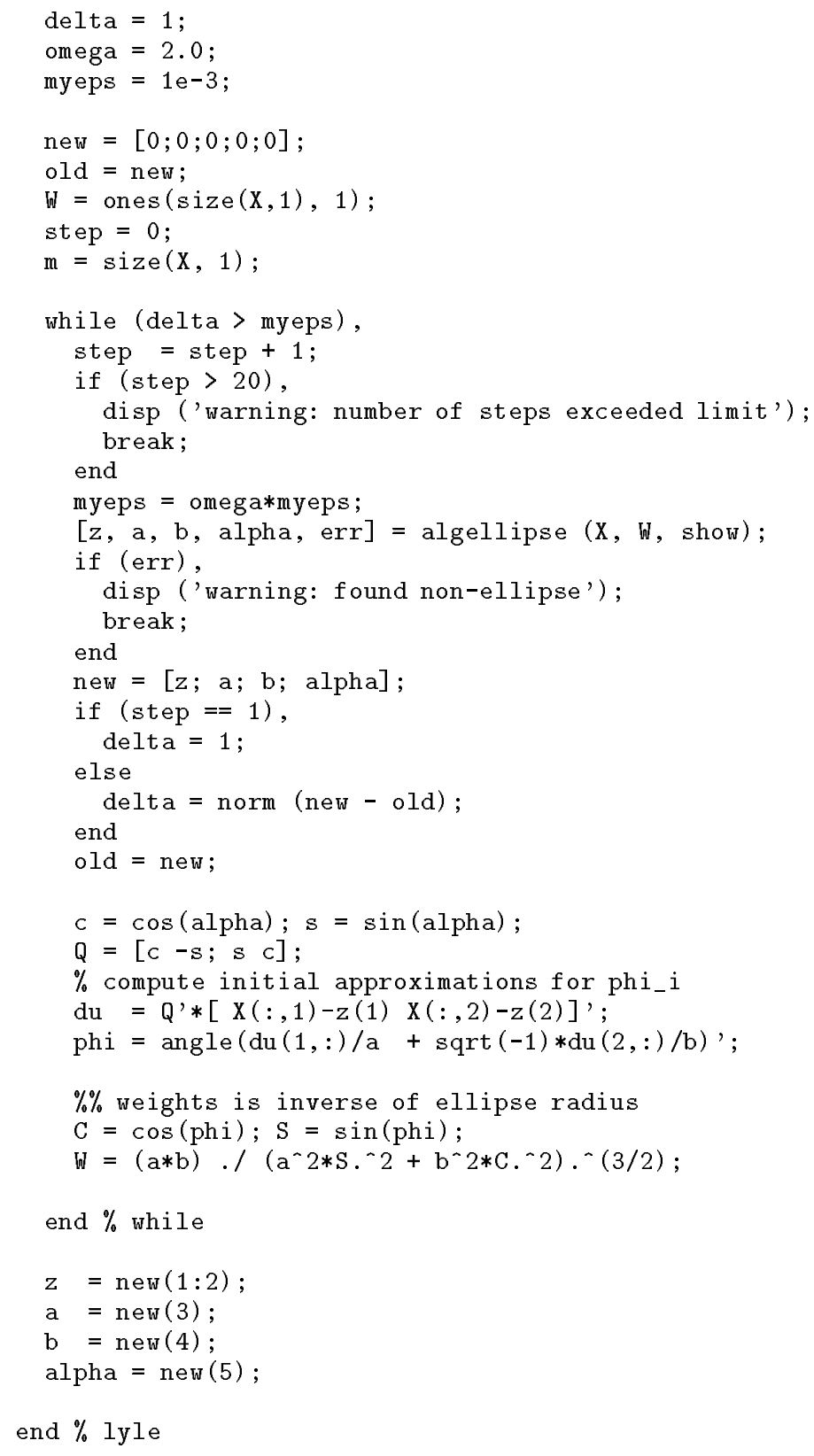

Geometric weights

function $[z, a, b$, alpha, step $]=$ wate 2 (X, show); 


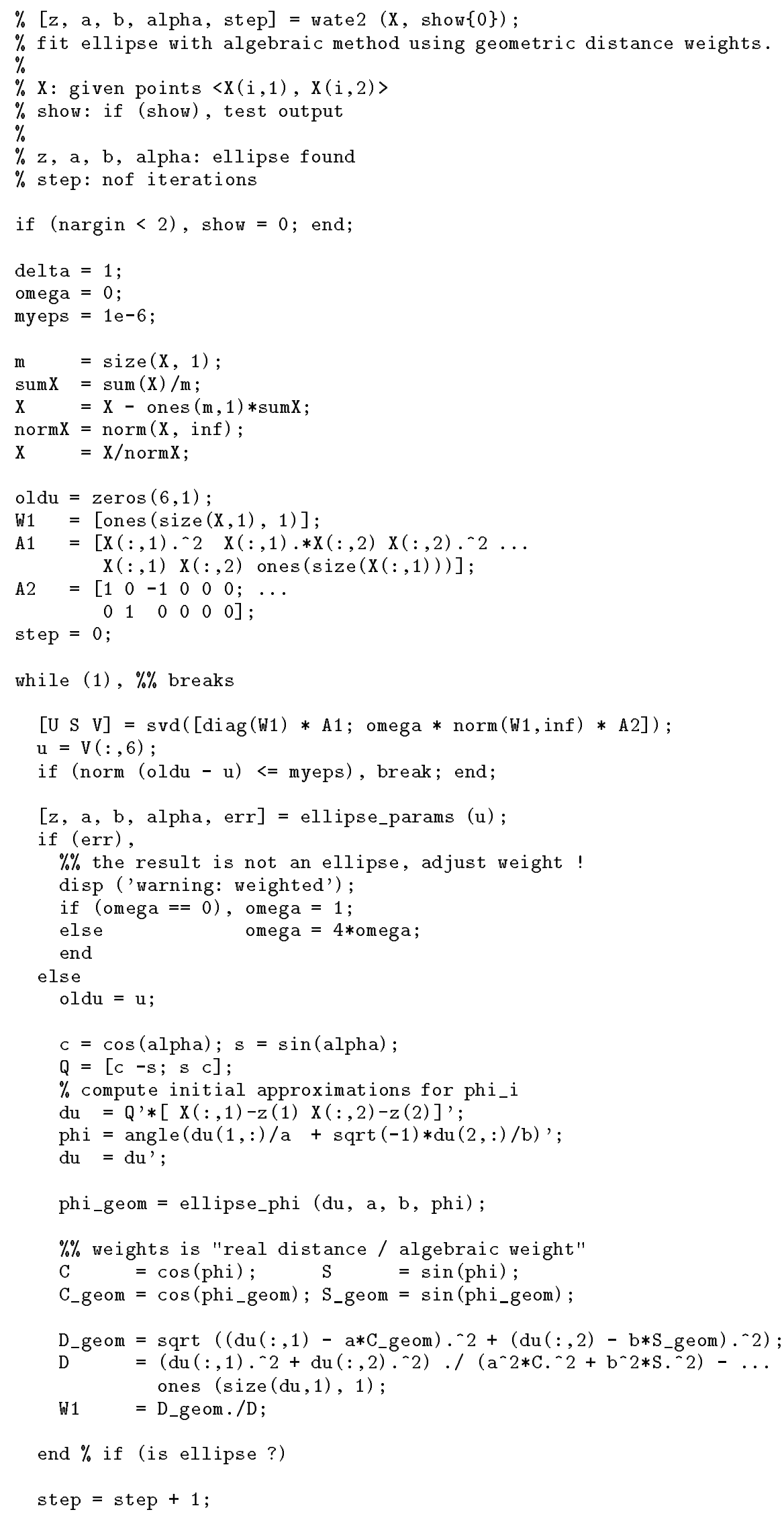




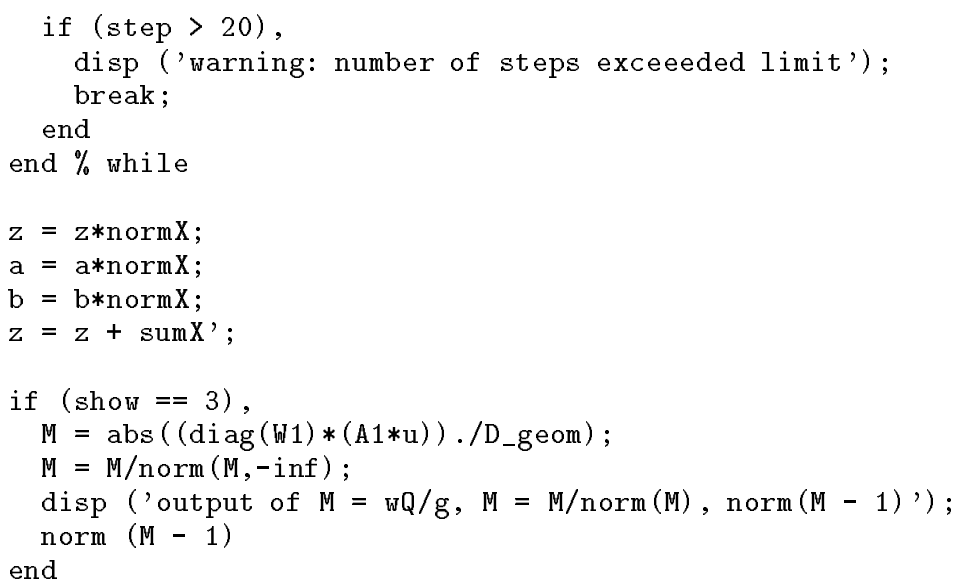

end $\%$ wate 2

\section{B.4 Geometric ellipse estimation}

\section{B.4.1 Utilities}

Parameter vector to parameter translation

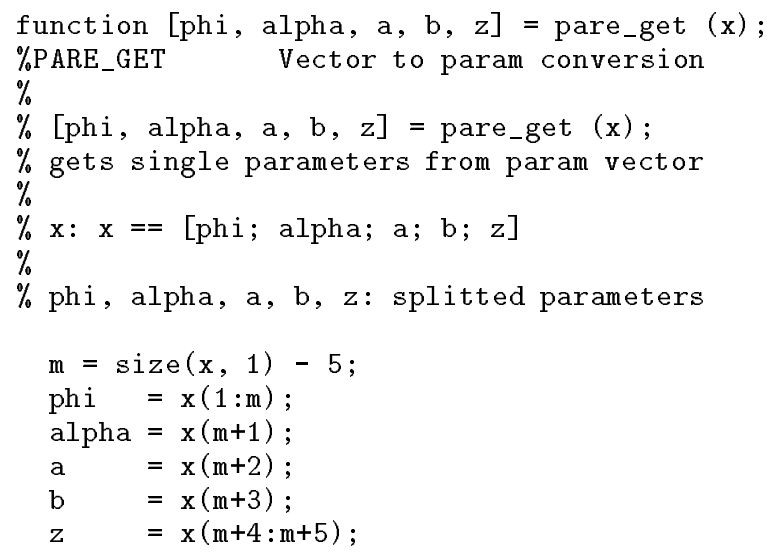

end $\%$ pare_get

\section{Parameter to parameter vector translation}

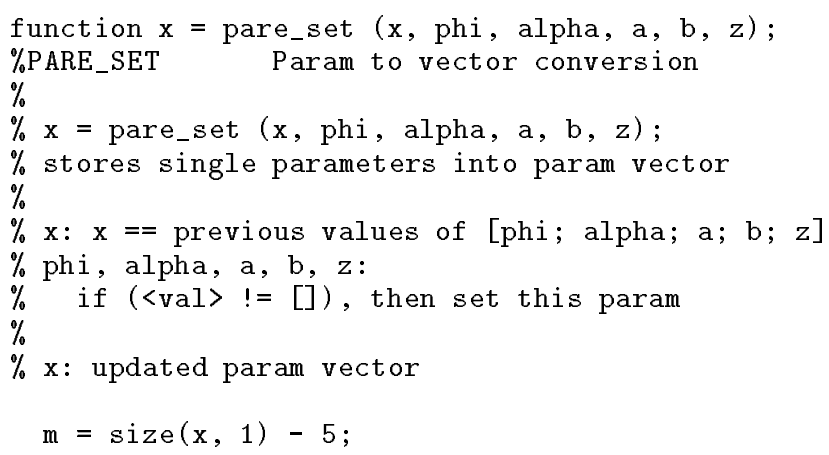




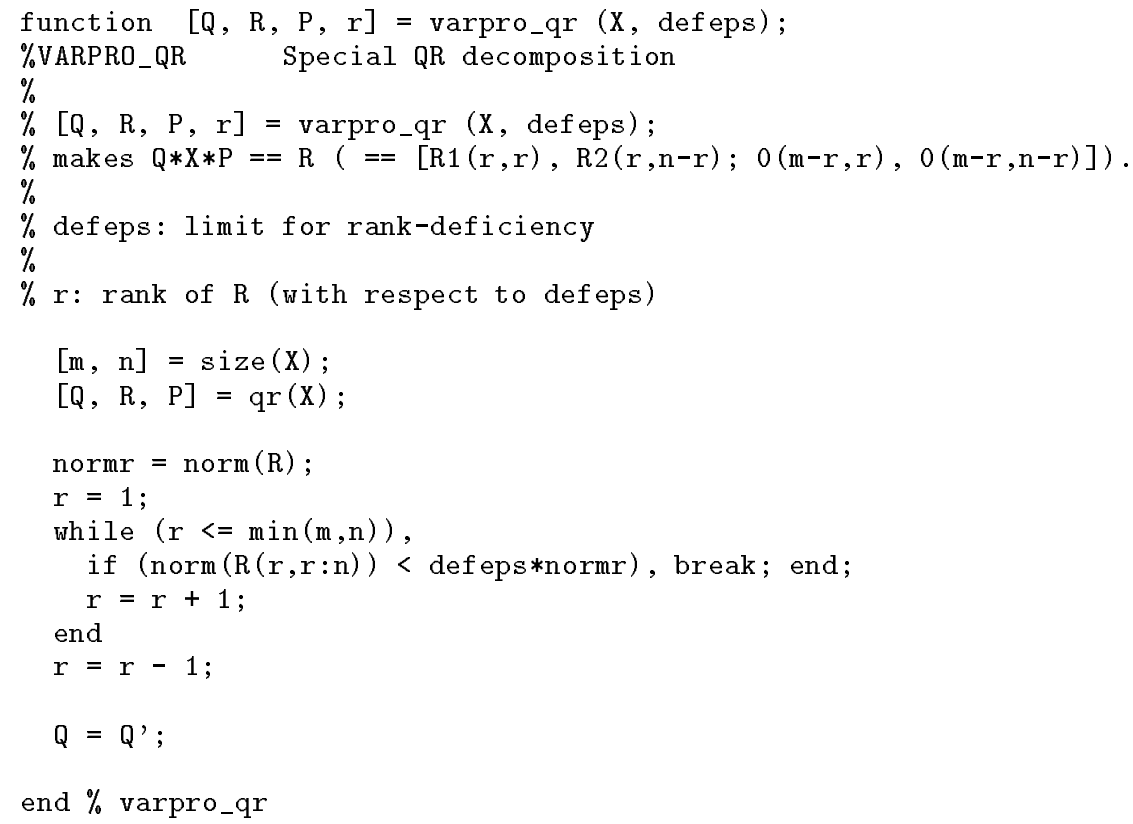

end $\%$ varpro_qr

\section{B.4.2 Gauss-Newton, Newton and Marquardt algorithms}

\section{Gauss-Newton iteration step}

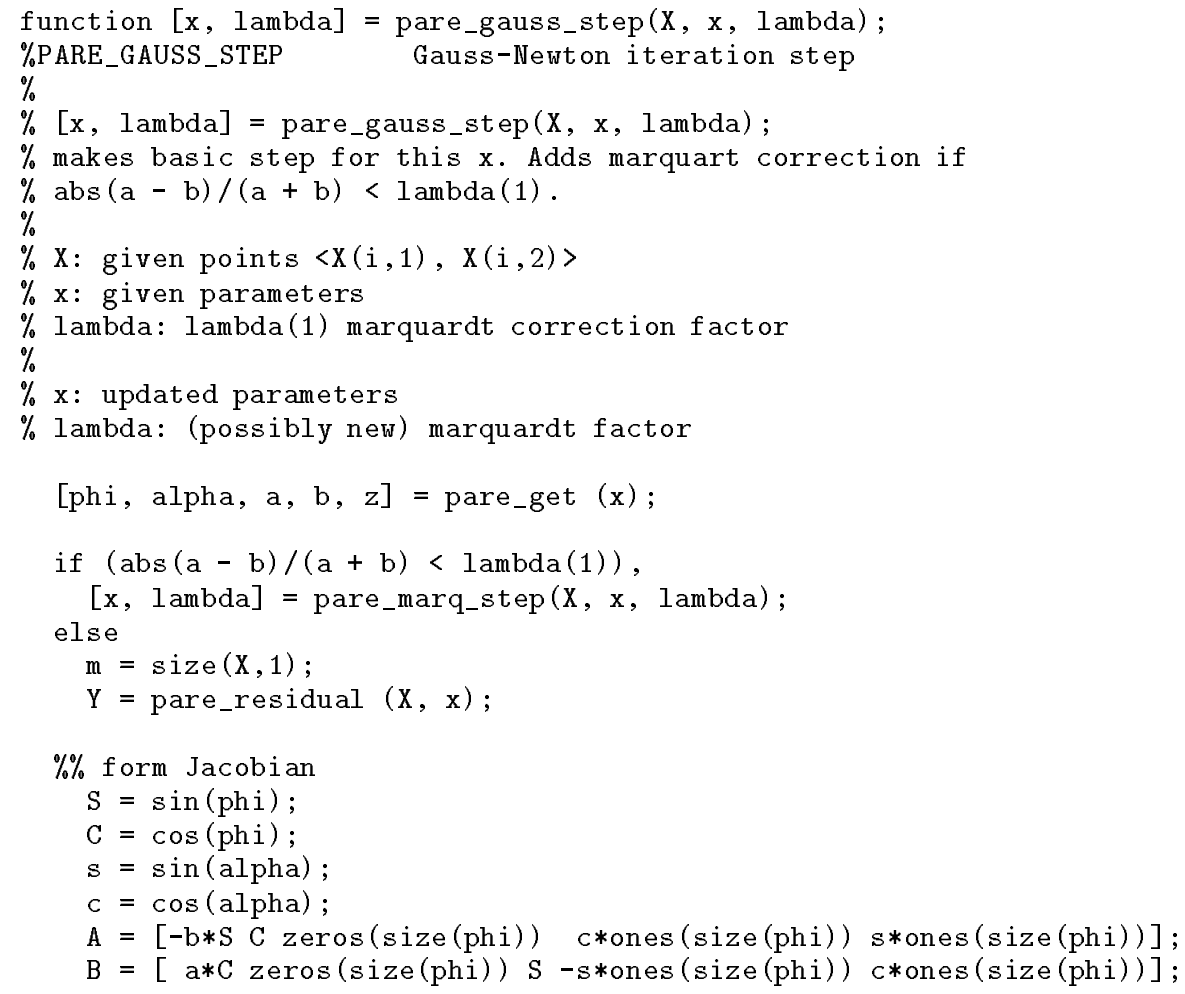




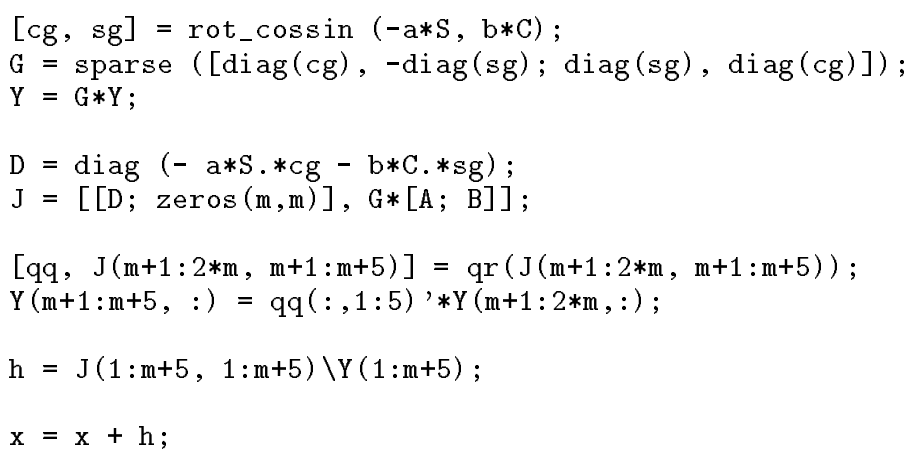

end $\%$ if (marq term necessary)

end \% pare_gauss_step

Newton iteration step

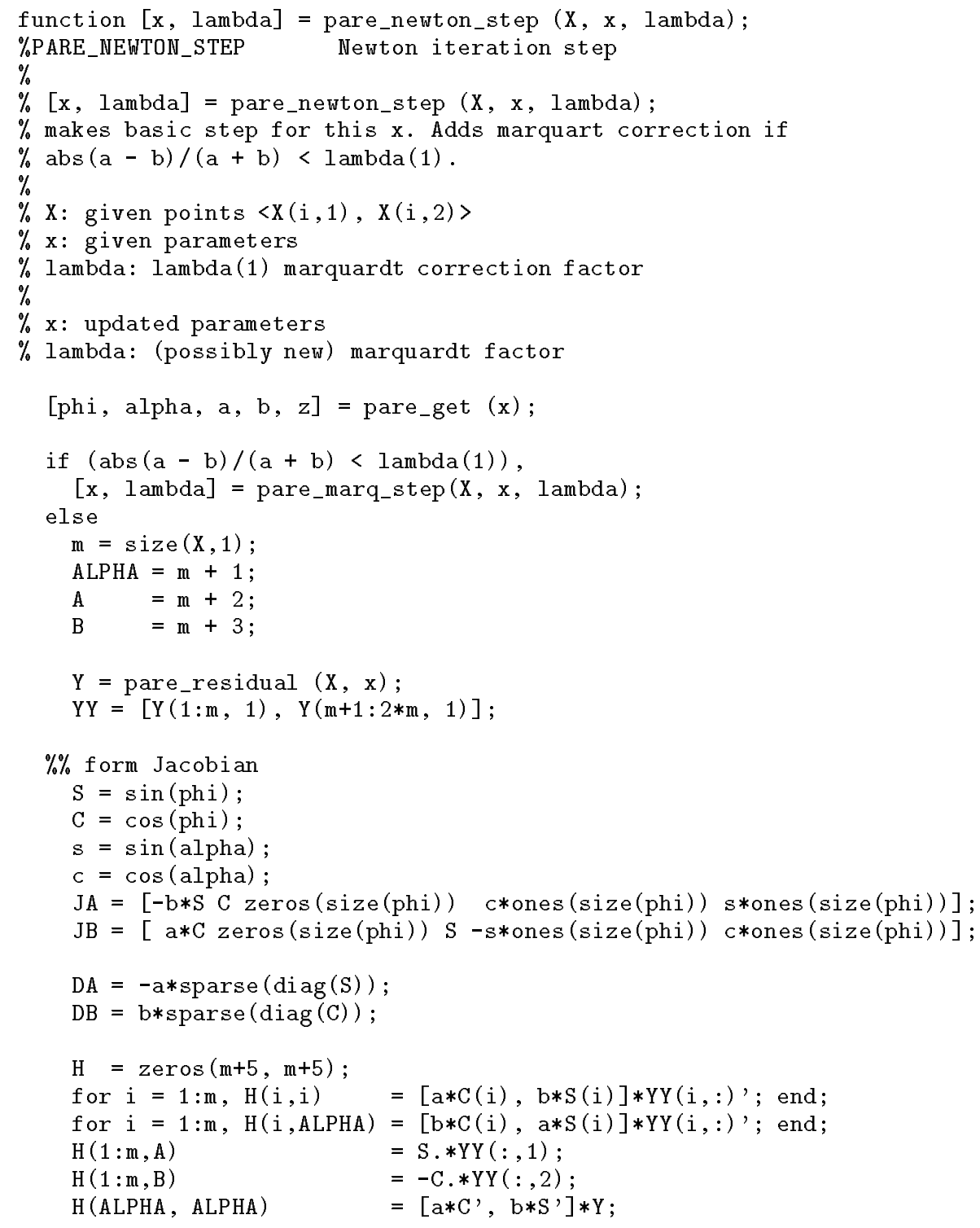




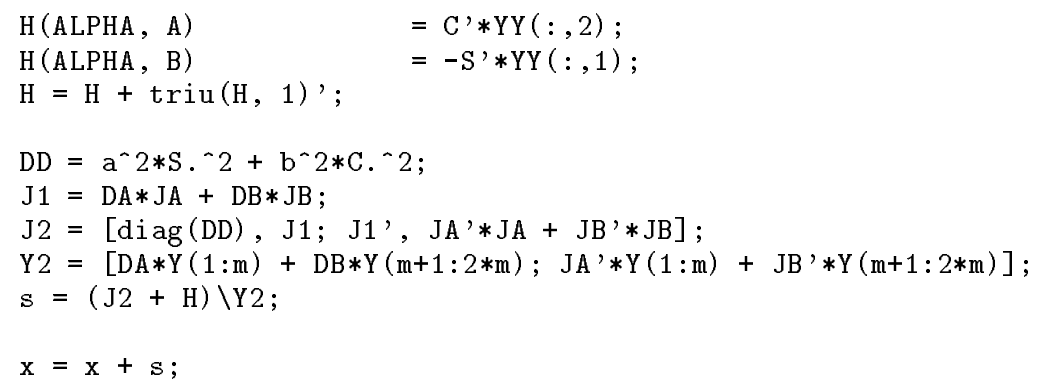

end \% if (marq term necessary)

end \% pare_newton_step

Gauss-Newton iteration step with Marquardt modification

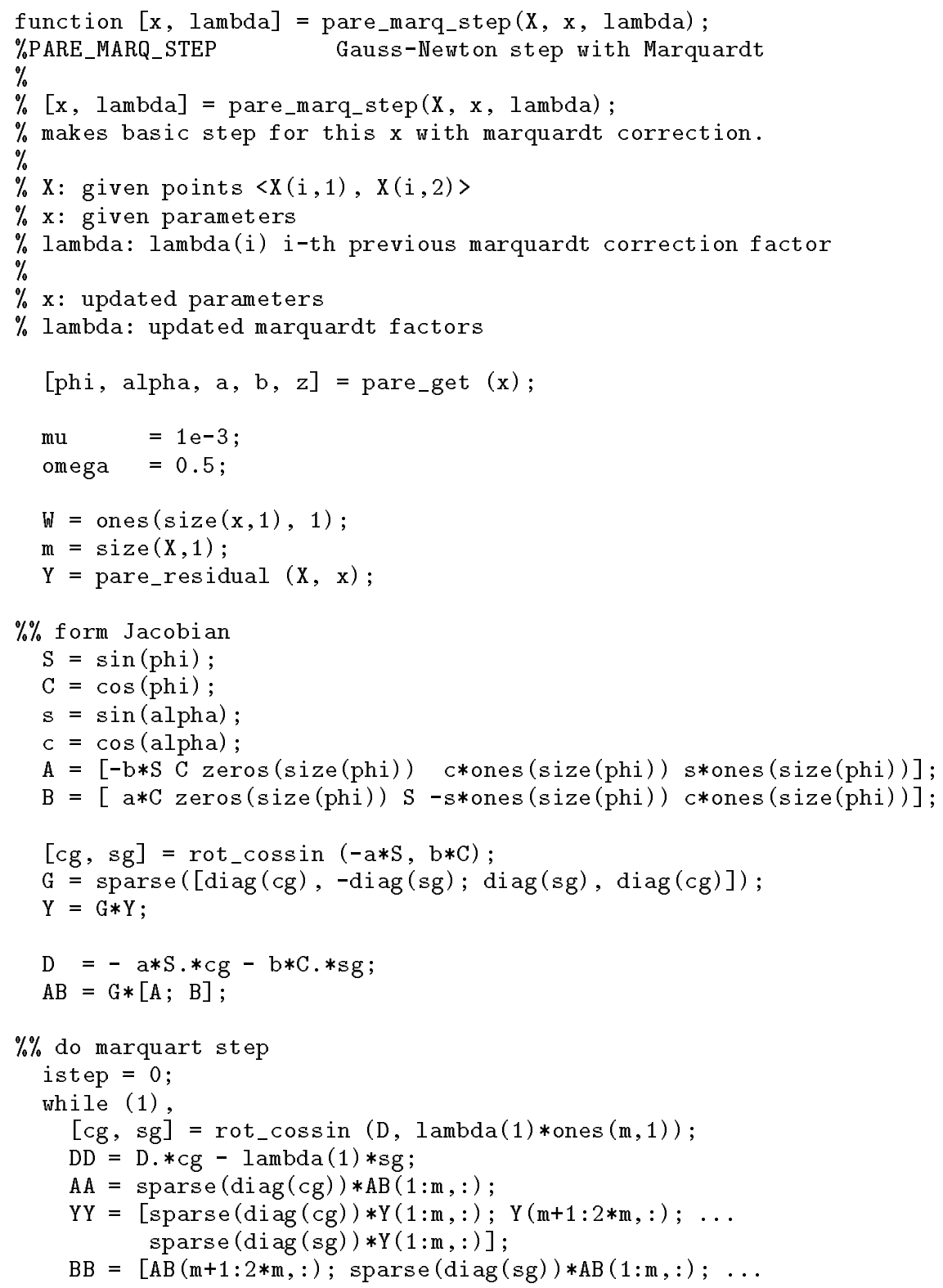




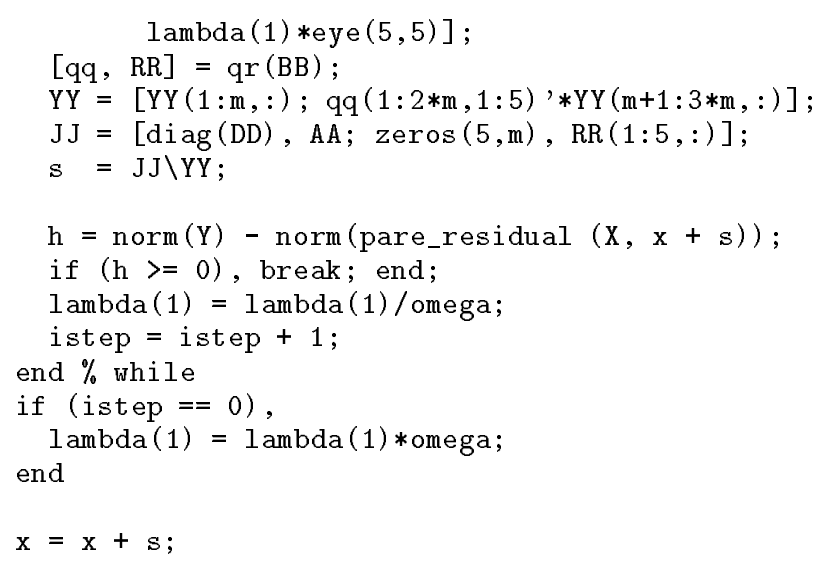

end \% pare_marq_step

Geometric estimation loop

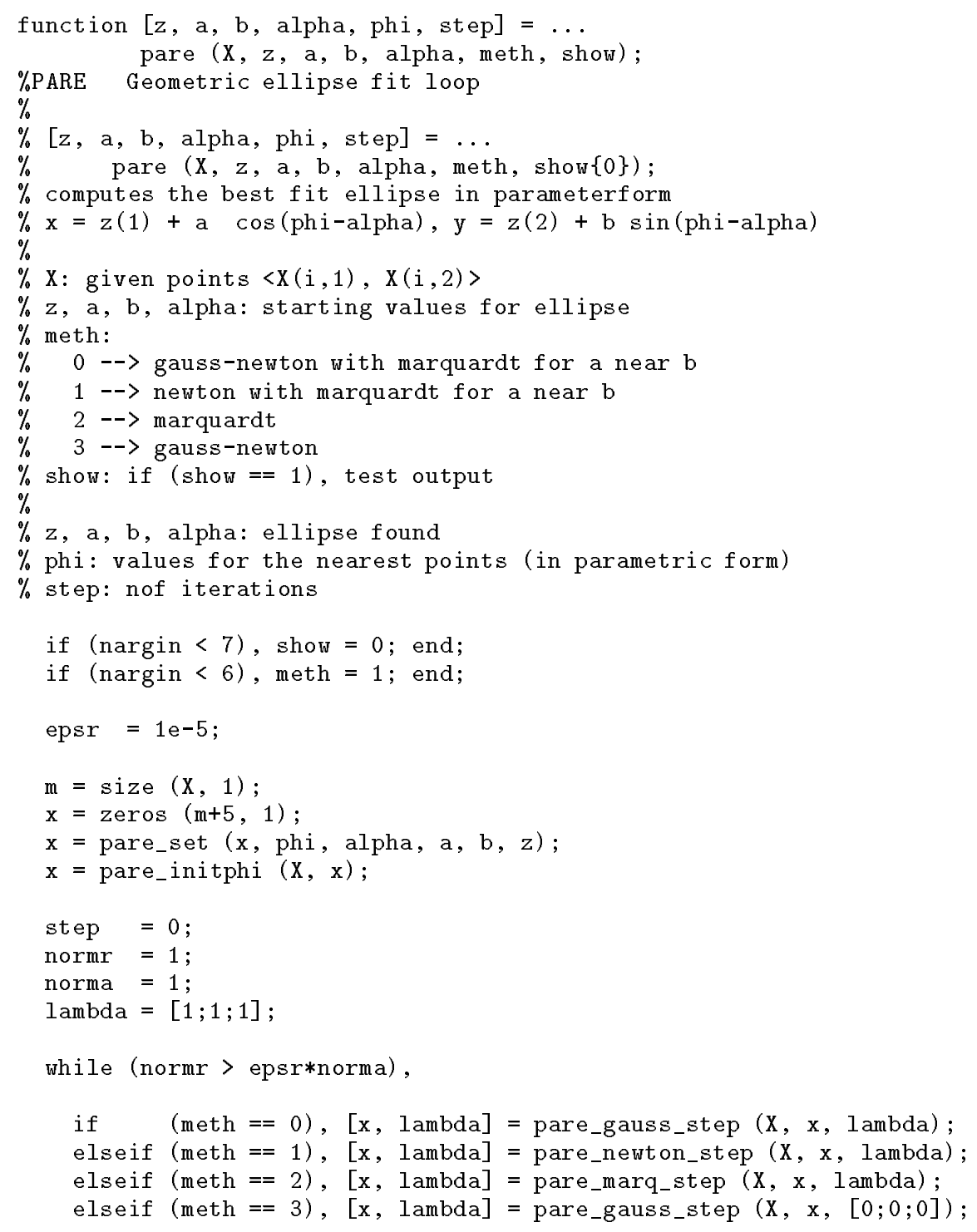




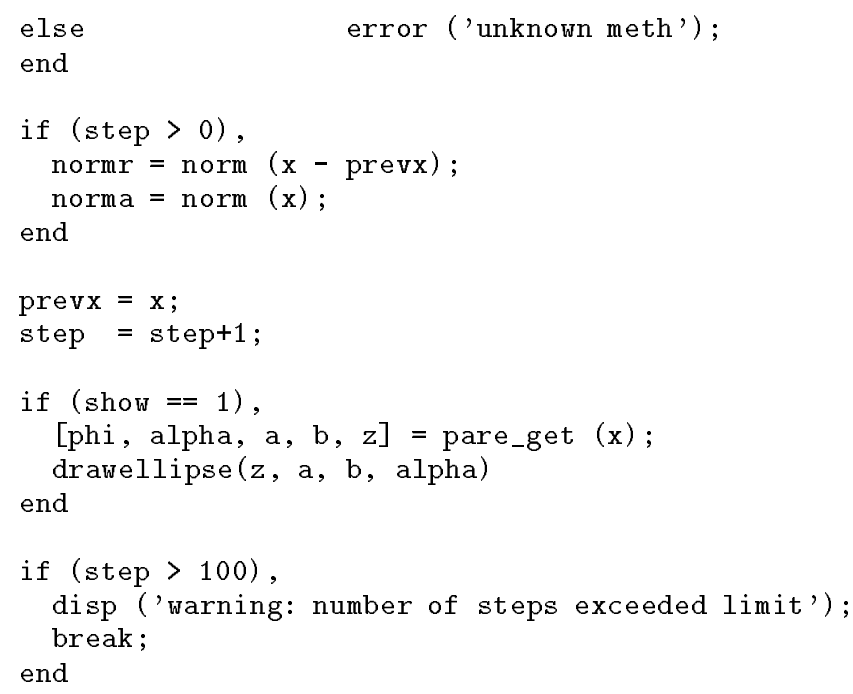

end \% while

$\left[\right.$ phi, alpha, a, b, z] $=\operatorname{pare}_{-}$get $(\mathrm{x})$;

end $\%$ pare

\section{B.4.3 The varpro algorithm}

The general varpro procedure

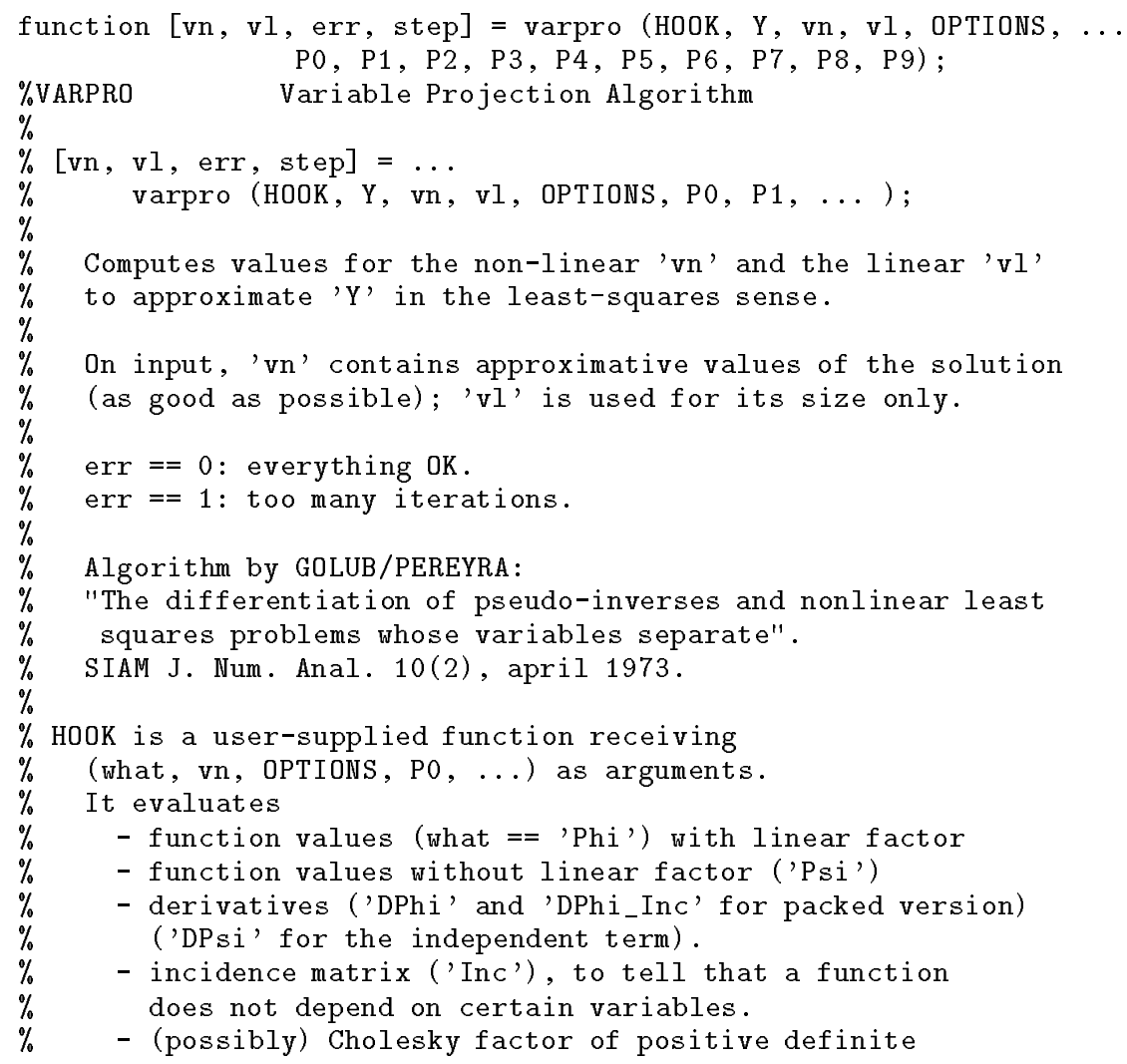




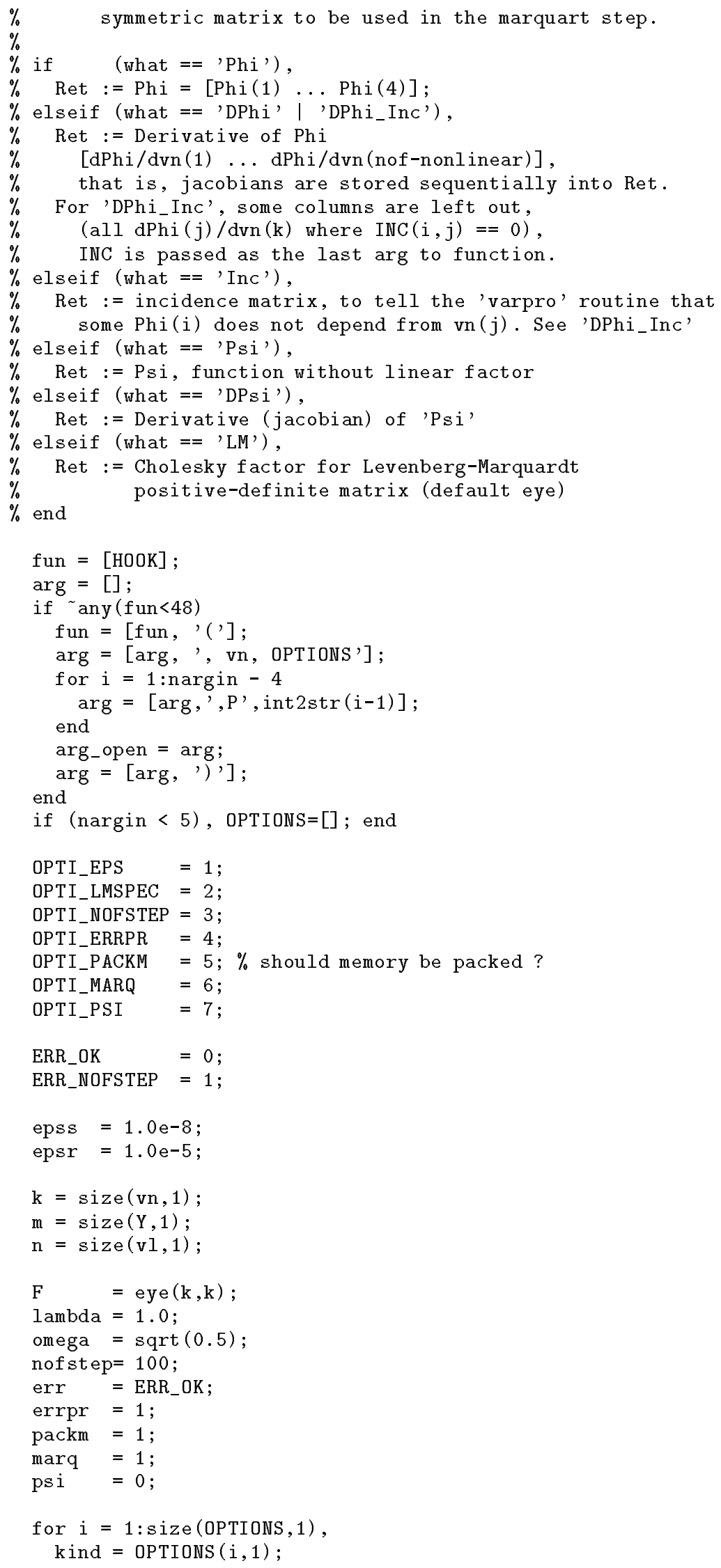




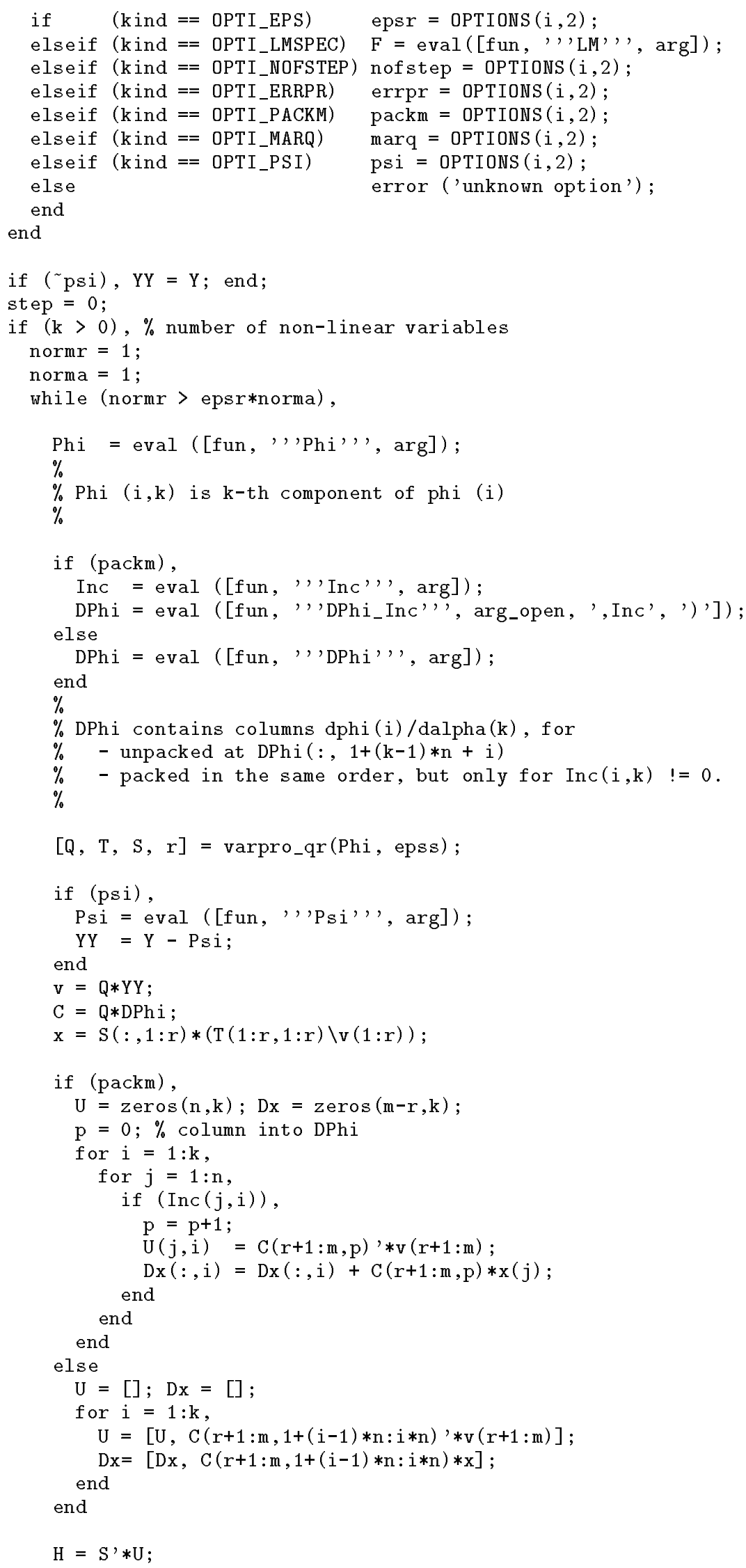




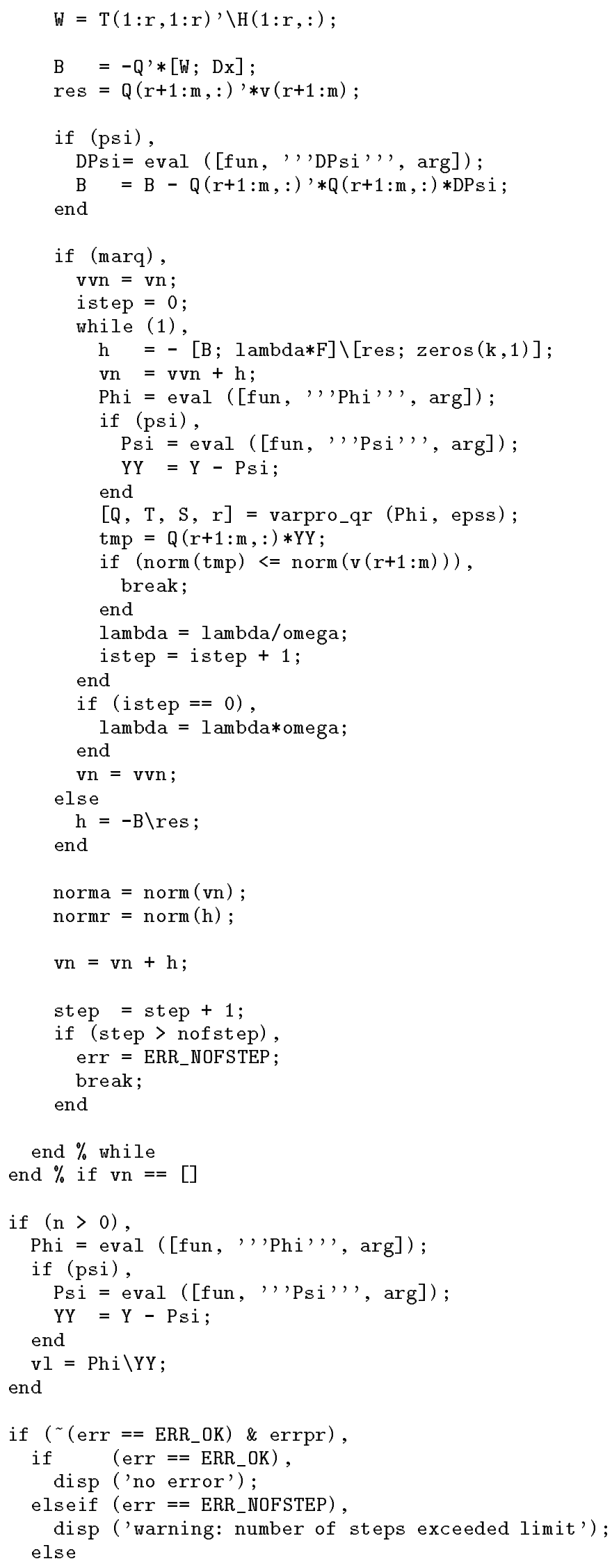


error ('fatal: illegal error number');

end

end

end $\%$ varpro

The varpro interface procedure

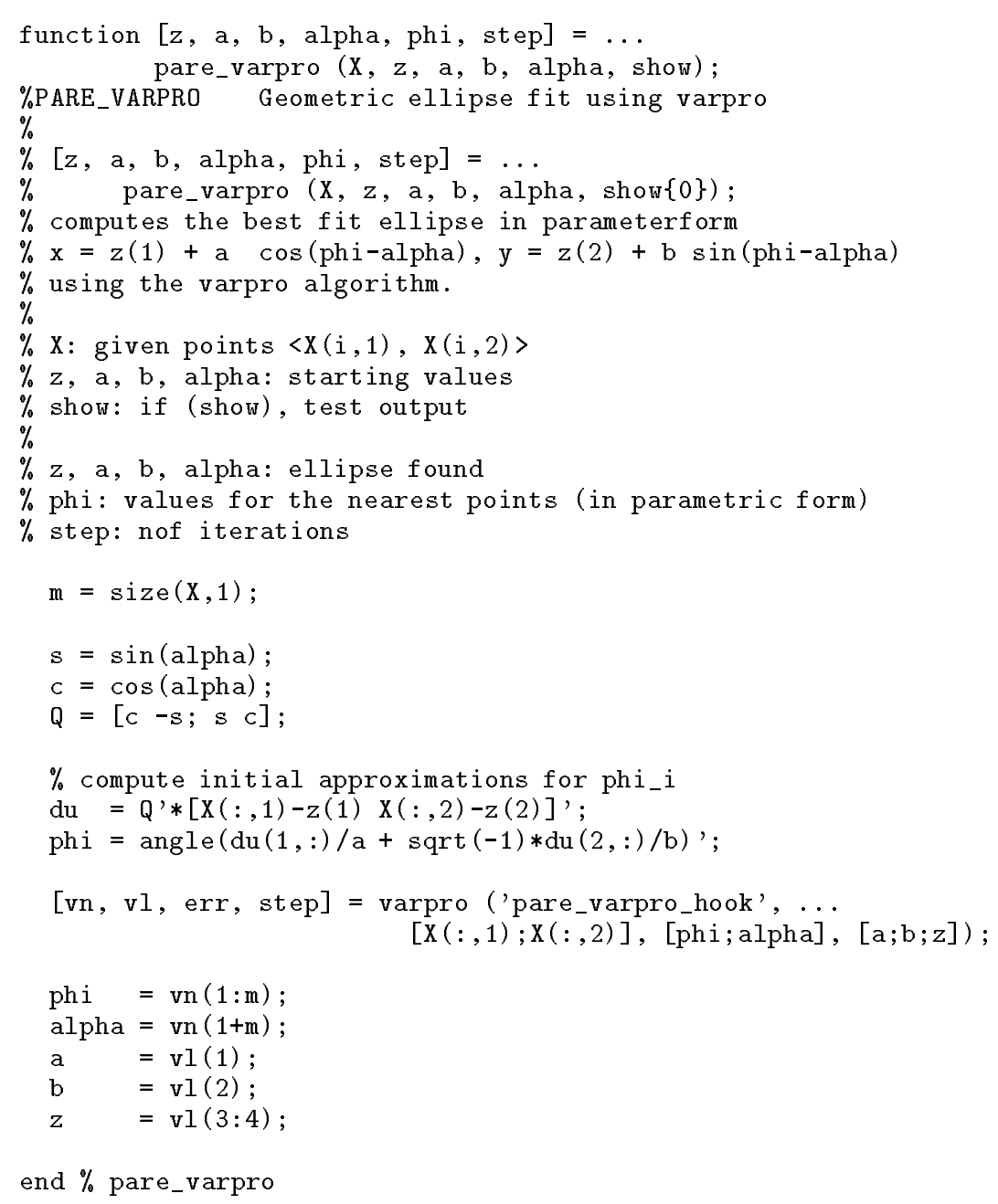

The varpro hook function

function Ret = pare_varpro_hook (what, vn, OPTIONS, INC);

$\%$ PARE_VARPRO_HOOK Hook for varpro ellipse fit

$\%$

$\%$ Ret = pare_varpro_hook (what, vn, OPTIONS, INC);

$\%$ Hook for the 'varpro' routine, returns data depending on 'what'.

$\%$ given data is $\mathrm{Y}=[\mathrm{x}-\operatorname{coord}(1: \mathrm{m}, 1) ; \mathrm{y}-\operatorname{coord}(1: \mathrm{m}, 1)]$

$\%$ estimation function is

$\% a * \operatorname{Phi}(1)+b * \operatorname{Phi}(2)+z(1) * \operatorname{Phi}(3)+z(2) * \operatorname{Ph} i(4)$

$\%$

$\%$ what: kind of data needed (see 'varpro')

$\%$ vn: non-linear parameters

$\%$ OPTIONS: same as options passed to 'varpro' routine

$\%$ INC: incidence matrix, for what == 'DPhi_Inc' only 


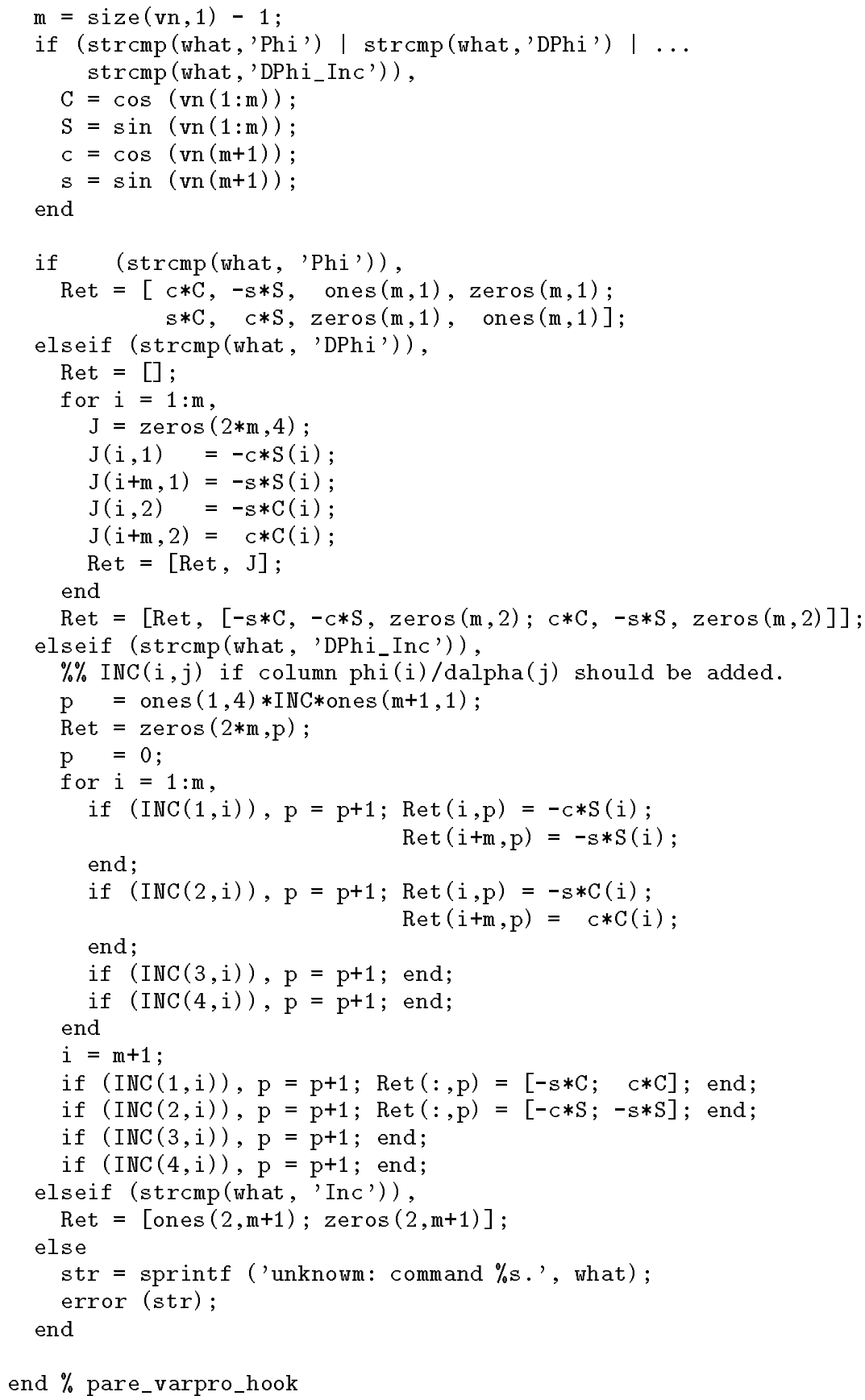

end \% pare_varpro_hook

\section{B.4.4 The odr algorithm}

The general odr procedure

function $[b$, delta, err, step $]=\ldots$

odr (HOOK, x, y, b, OPTIONS, $\mathrm{w}, \mathrm{D}$, delta, ...

P0, P1, P2, P3, P4, P5, P6, P7, P8, P9); 


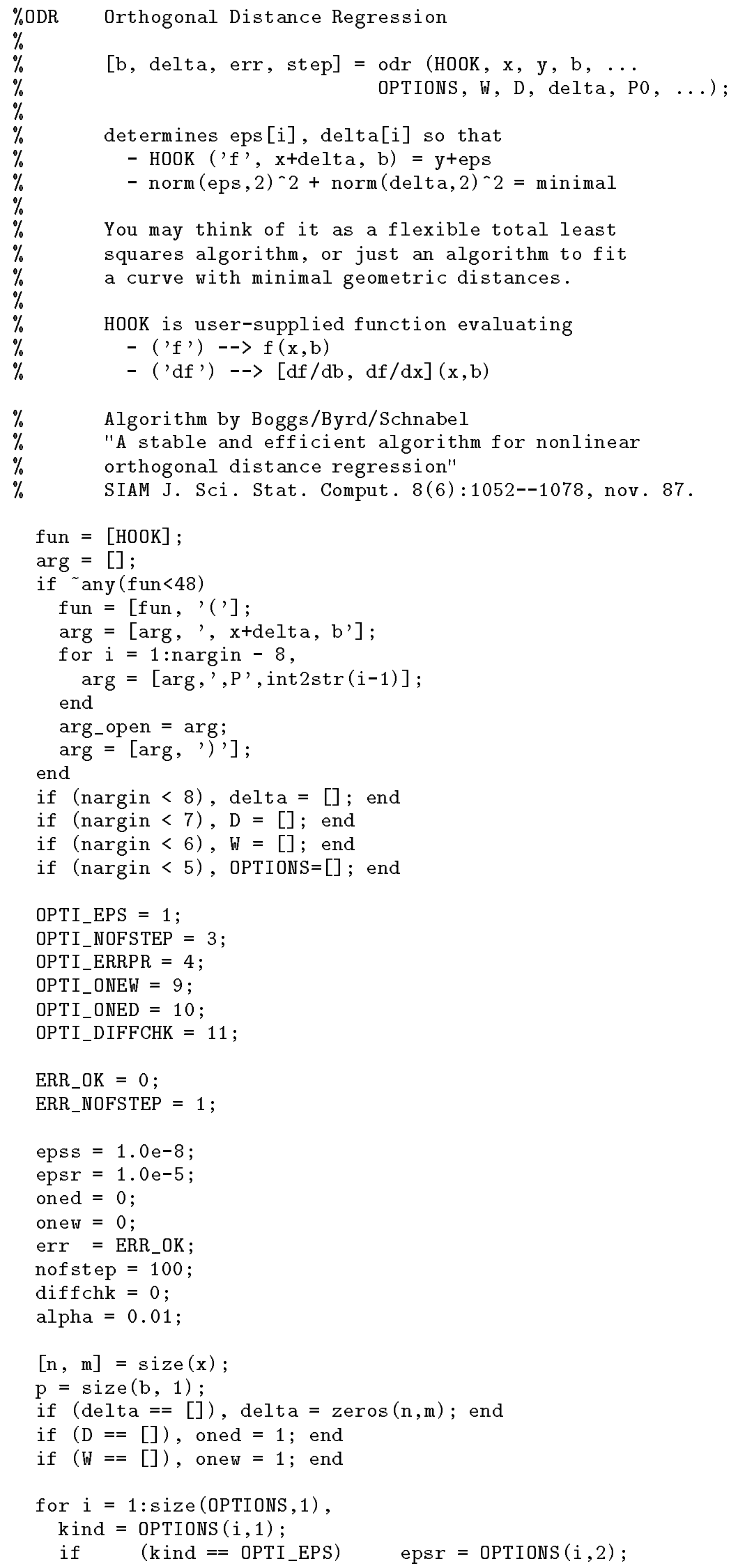




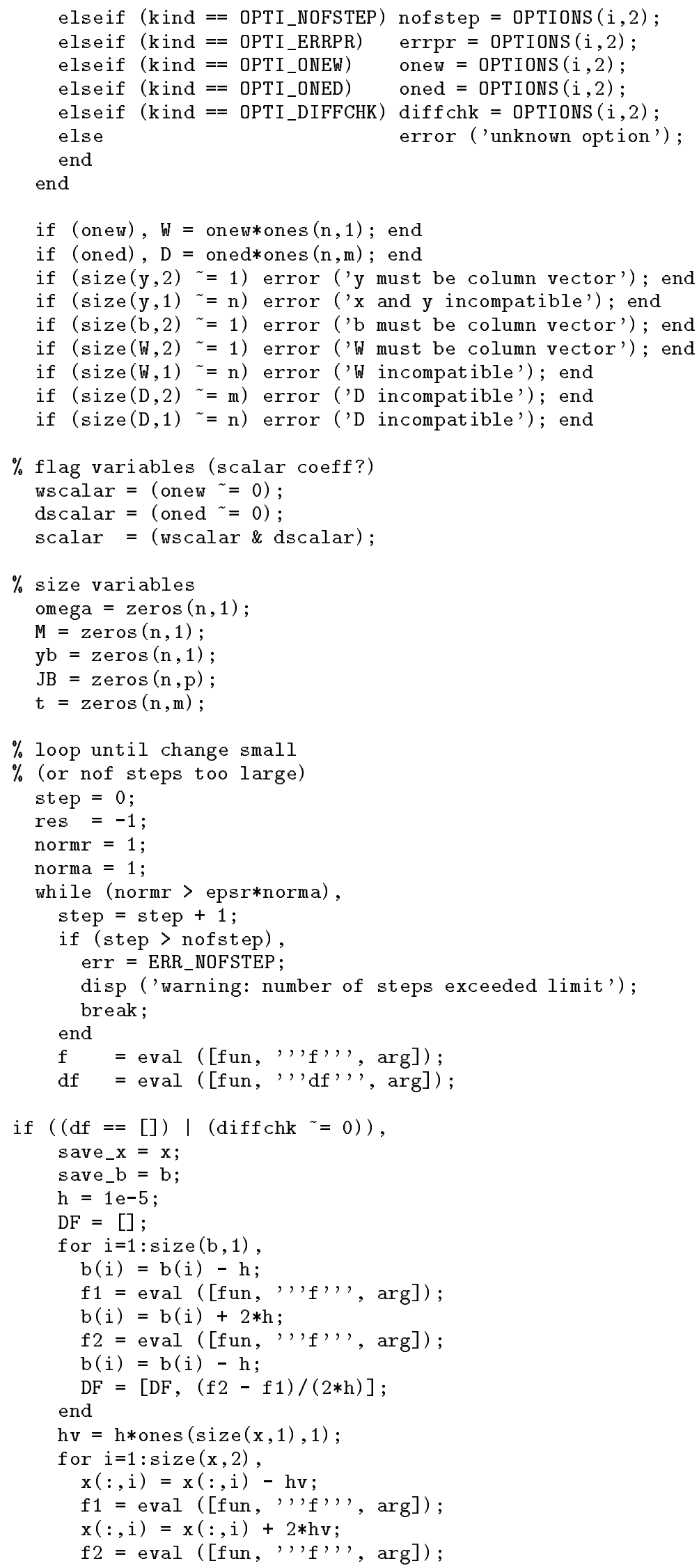




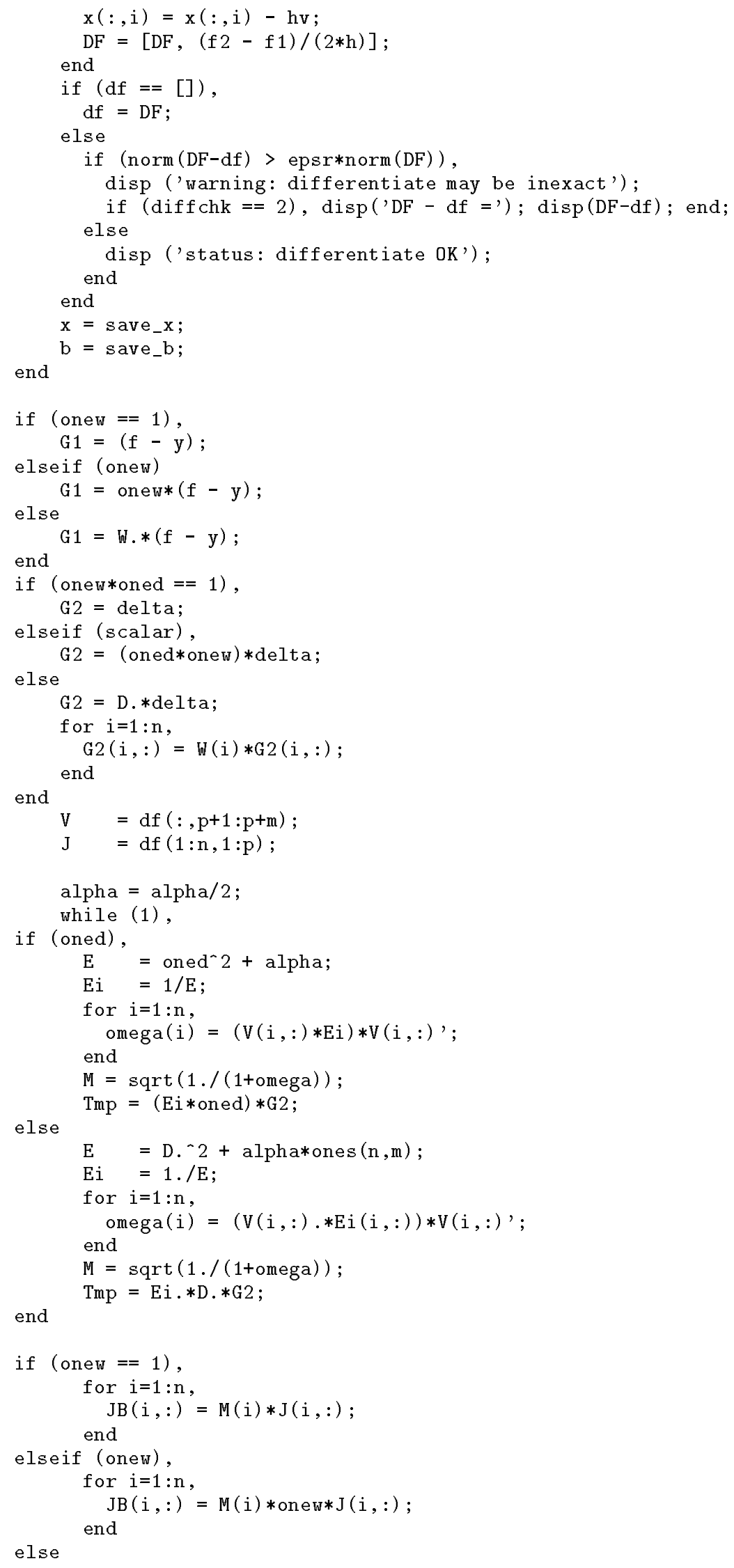




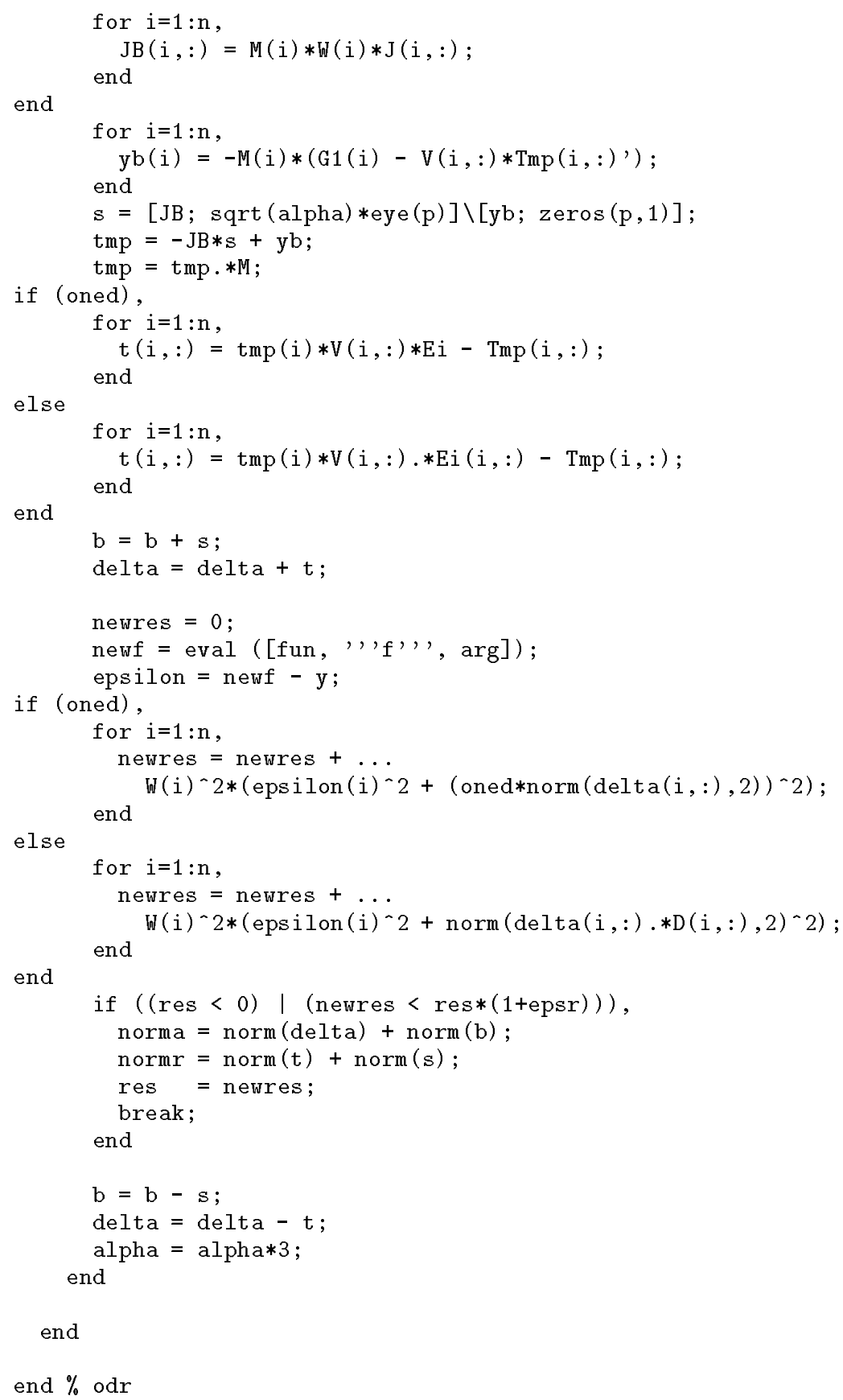

The odr interface procedure

function $[z, a, b$, alpha, step $]=\ldots$

alge_odr (X, z, a, b, alpha, show); $\%$ ALGE_ODR

$\%$

$\% \quad[z, a, b, a l p h a$, step $]=\ldots$

$\% \quad$ alge_odr (X, z, a, b, alpha, show);

$\% \quad$ computes the best fit ellipse in parameterform

$\% \quad x=z(1)+a \cos (p h i-a l p h a), y=z(2)+b \sin (p h i-a l p h a)$

$\%$ using the odr algorithm. \% 


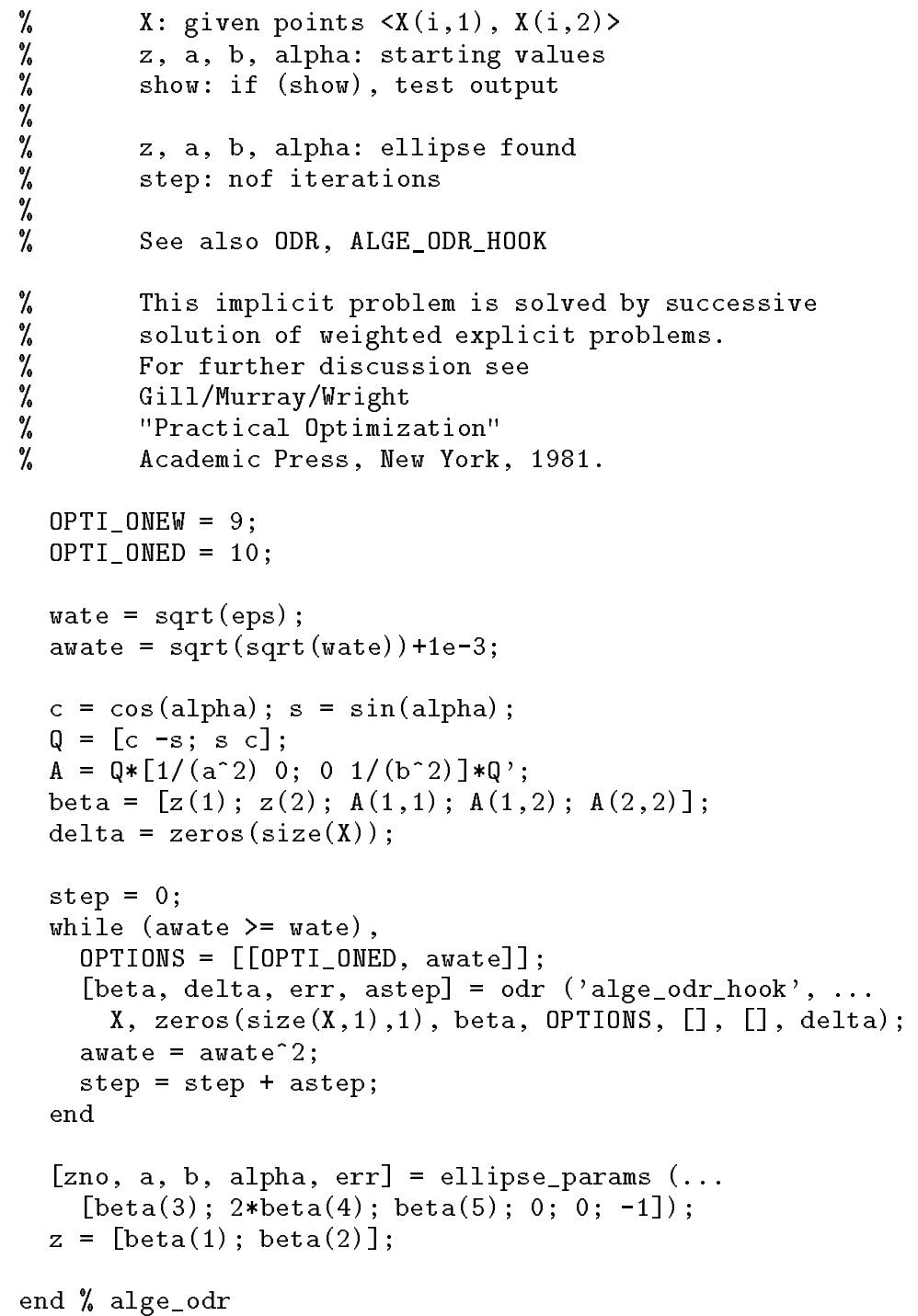

The odr hook function

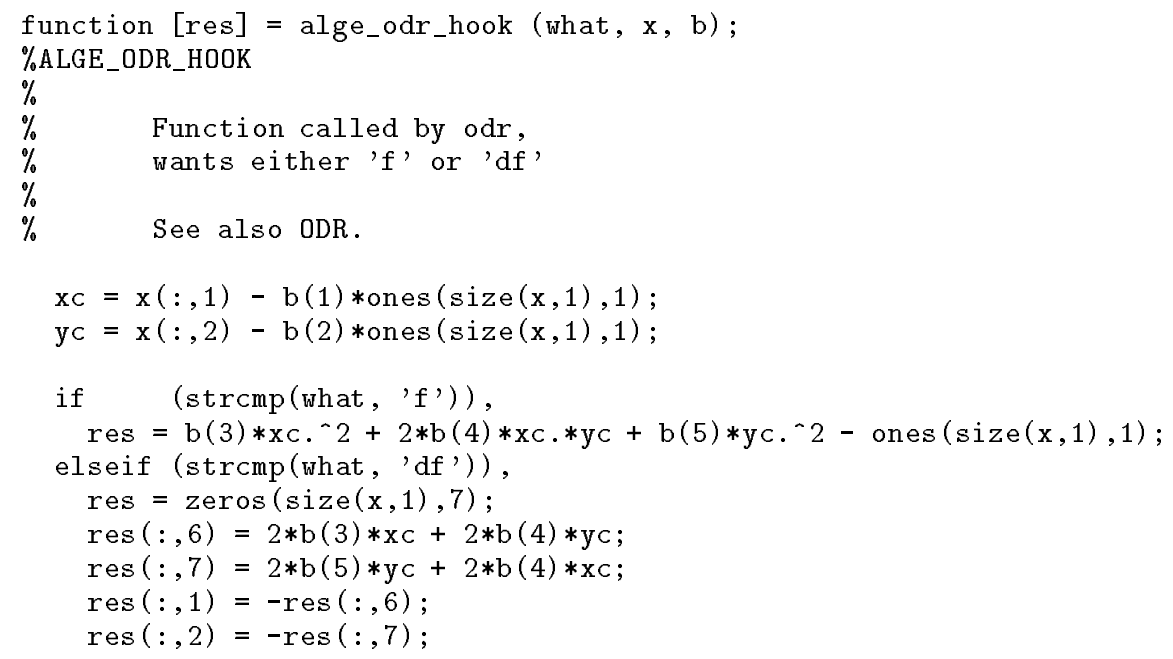




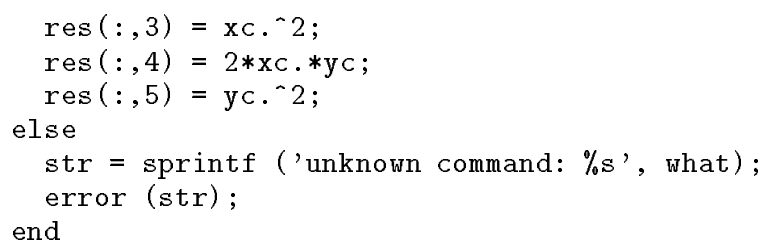

end \% alge_odr_hook

\section{Data sets}

This section lists the data sets used in the paper.

\section{C.1 Various data sets}

Data for eccentric ellipse

\begin{tabular}{|c|c|}
\hline$=[$ & \\
\hline 1.9400 & 3.7540 \\
\hline 2.8640 & 4.7500 \\
\hline 4.9420 & 6.6280 \\
\hline 5.6360 & 8.1520 \\
\hline 6.5120 & 9.2080 \\
\hline 8.4060 & 10.6160 \\
\hline 9.3760 & 11.5540 \\
\hline 10.5780 & 12.8440 \\
\hline 11.5940 & 14.9560 \\
\hline 13.8100 & 16.2460 \\
\hline 16.3520 & 16.5980 \\
\hline 16.2120 & 13.7240 \\
\hline 15.3340 & 12.4920 \\
\hline 14.7340 & 11.4960 \\
\hline 12.0100 & 9.5600 \\
\hline 10.5780 & 8.2120 \\
\hline 9.3760 & 7.0380 \\
\hline 9.3760 & 7.0380 \\
\hline 7.5760 & 4.3980 \\
\hline 5.8200 & 3.9300 \\
\hline 4.4800 & 2.9320 \\
\hline 3.0020 & 2.6980 \\
\hline
\end{tabular}

Points near ellipse

\begin{tabular}{|c|c|}
\hline$r=[$ & \\
\hline 2.0143 & 10.5575 \\
\hline 17.3465 & 3.2690 \\
\hline-8.5257 & -7.2959 \\
\hline-7.9109 & -7.6447 \\
\hline 16.3705 & -3.8815 \\
\hline-15.3434 & 5.0513 \\
\hline-21.5840 & -0.601 \\
\hline 9.4111 & -9.0697 \\
\hline
\end{tabular}




\section{C.2 Comparison of the geometric estimation algorithms}

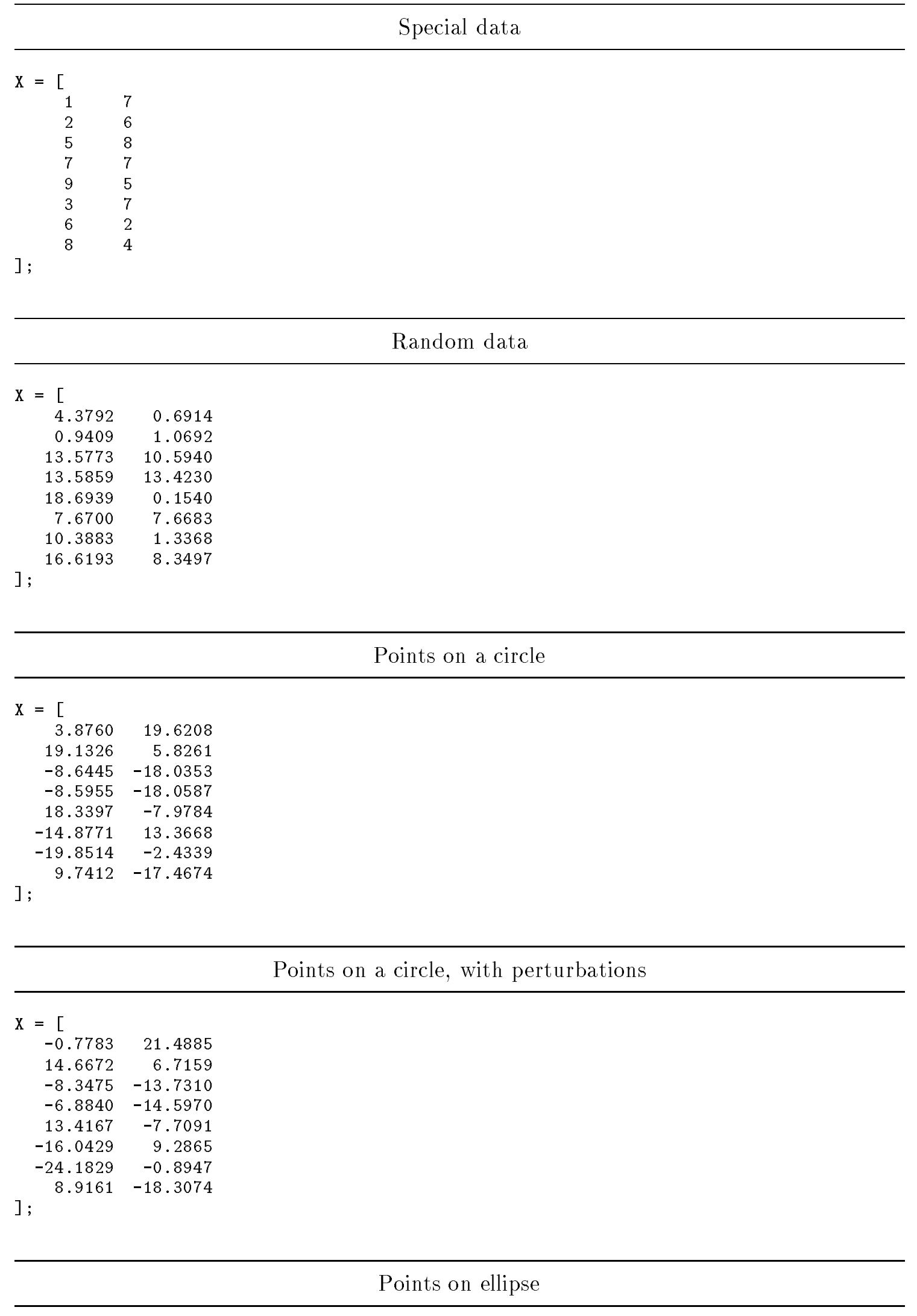




\begin{tabular}{|c|c|}
\hline$=[$ & \\
\hline 3.8760 & 9.8104 \\
\hline 19.1326 & 2.9130 \\
\hline-8.6445 & -9.0177 \\
\hline-8.5955 & -9.0294 \\
\hline 18.3397 & -3.989 \\
\hline-14.8771 & 6.6834 \\
\hline-19.8514 & -1.216 \\
\hline 9.7412 & -8.733 \\
\hline
\end{tabular}

Points on ellipse, with perturbations

\begin{tabular}{|c|c|}
\hline \multicolumn{2}{|l|}{$X=[$} \\
\hline-0.7783 & 11.6781 \\
\hline 14.6672 & 3.8028 \\
\hline-8.3475 & -4.7133 \\
\hline-6.8840 & -5.5677 \\
\hline 13.4167 & -3.7199 \\
\hline-16.0429 & 2.6031 \\
\hline-24.1829 & 0.3222 \\
\hline 8.9161 & -9.5737 \\
\hline
\end{tabular}

Points on hyperbola branch

$\begin{array}{rr}X=[ & \\ 20.0658 & -0.8118 \\ 20.1711 & -1.3107 \\ 20.0266 & 0.5163 \\ 20.0268 & 0.5176 \\ 20.1575 & 1.2575 \\ 20.0113 & -0.3362 \\ 20.0003 & 0.0560 \\ 20.0913 & 0.9564 \\ ] ; & \end{array}$

Points on hyperbola branch, with perturbations

\begin{tabular}{|c|c|}
\hline \multicolumn{2}{|l|}{$X=[$} \\
\hline 15.4115 & 1.0559 \\
\hline 15.7057 & -0.4209 \\
\hline 20.3236 & 4.8207 \\
\hline 21.7383 & 3.9792 \\
\hline 15.2345 & 1.5268 \\
\hline 18.8455 & -4.4166 \\
\hline 15.6687 & 1.5952 \\
\hline 19.2661 & 0.1164 \\
\hline
\end{tabular}

